\title{
Industry/Government Open Systems Specification Testing Framework
}

Dr. Jean-Philippe Favreau Editor

U.S. DEPARTMENT OF COMMERCE Tecrinology Administration

National Institute of Standards and Technology

IGOSS Testing Program

Computer Systems Laboratory

Gaithersburg, MD 20899

June 1994 - Version 1.0

QC

100

.456

NLT

\# 5438 



\section{Industry/Government Open Systems Specification Testing Framework}

Dr. Jean-Philippe Favreau Editor

U.S. DEPARTMENT OF COMMERCE Technology Administration National Institute of Standards and Technology IGOSS Testing Program Computer Systems Laboratory Gaithersburg, MD 20899

June 1994 - Version 1.0

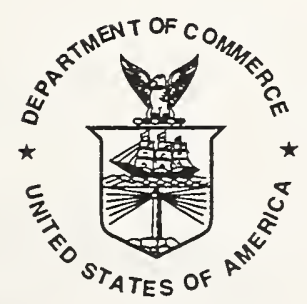

U.S. DEPARTMENT OF COMMERCE Ronald H. Brown, Secretary

TECHNOLOGY ADMINISTRATION

Mary L. Good, Under Secretary for Technology

NATIONAL INSTITUTE OF STANDARDS

AND TECHNOLOGY

Aratl Prabhakar, Dlrector 



\title{
TESTING FRAMEWORK
}

Version 1.0

June 1, 1994

\author{
Contributors Include: \\ Dr. Jean-Philippe Favreau, NIST \\ Stephen Nightingale, NIST \\ Brent Kempton, JITC \\ Ken Thomas, JITC
}

Editor: Dr. Jean-Philippe Favreau

Computer Systems Laboratory

National Institute of Standards and Technology

Gaithersburg, MD 20899

USA 



\section{TABLE OF CONTENTS}

OVERVIEW.

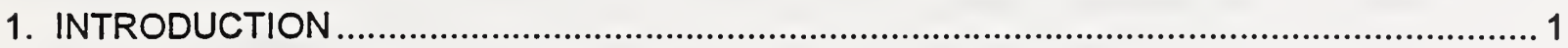

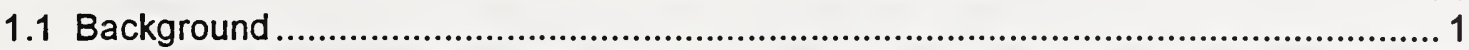

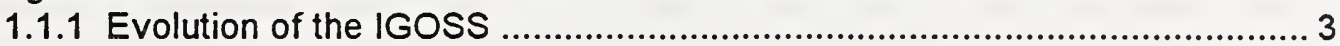

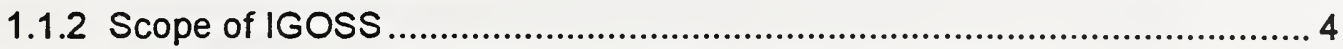

1.1.3 Relationship of the IGOSS to Existing Profile Documents ......................... 5

1.1.4 Applicability .........................................................................................

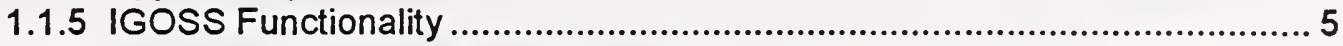

1.1.6 Sources of Protocols Specification ............................................................ 6

1.1.6.1 Primary Source .......................................................................... 6

1.1.6.2 Secondary Sources ...................................................................

1.1.6.3 Tertiary Sources ..................................................................... 7

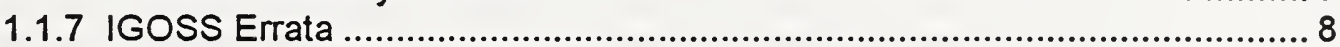

1.2 Purpose of IGOSS Testing Framework ............................................................... 8

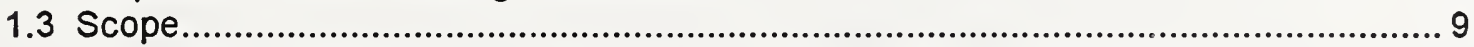

1.4 Overview of Testing ............................................................................................... 9

1.5 Definitions ........................................................................................................... 11

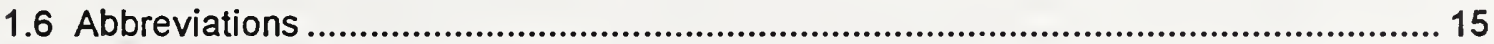

2. ORGANIZATIONAL MODEL …………............................................................................. 16

2.1 Program Sponsor/Authority/Agent ...................................................................... 18

2.2 IGOSS Accreditation Authority ........................................................................... 19

2.3 Suppliers of the Means of Testing ...................................................................... 19

2.4 Conformance Test Laboratories .......................................................................... 19

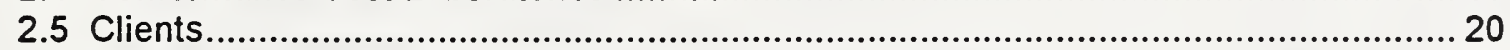

2.6 Criteria for Registration of a Conformant IGOSS Product ......................................... 20

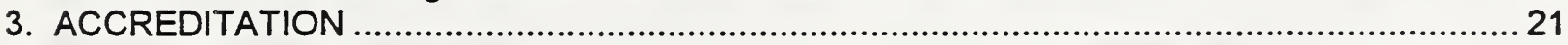

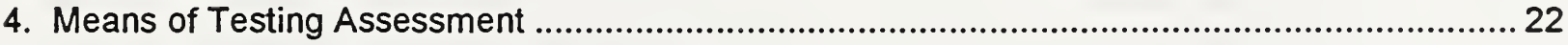

4.1 Registered Abstract Test Suites......................................................................... 22

4.2 Means of Testing Supplier ..................................................................................... 23

4.3 Approved MOT Assessment Laboratory …………................................................... 23

4.4 MOT Registration Authority .................................................................................... 24

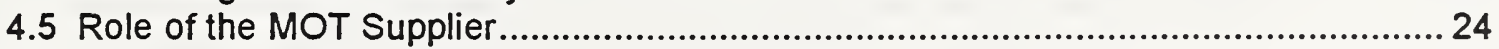

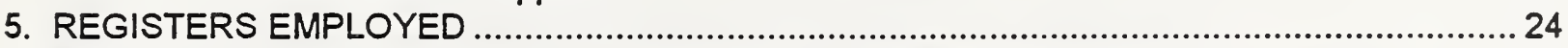

6. EMPLOYMENT OF THE OSI CONFORMANCE TESTING METHODOLOGY ..........................2 26

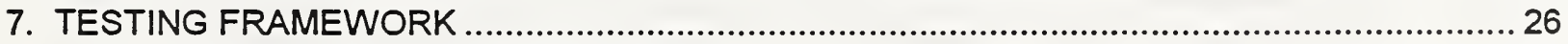

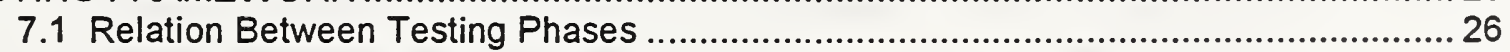

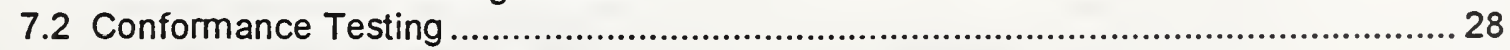

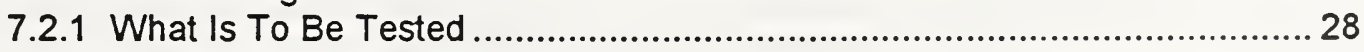

7.2.2 How Testing Is Conducted ................................................................... 29

7.2.2.1 Testing Elements.................................................................... 29

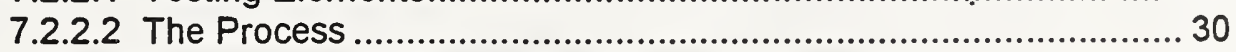

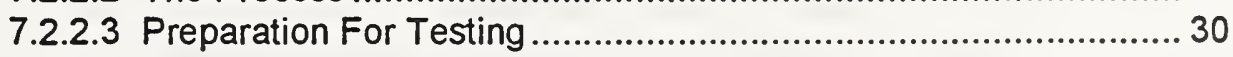

7.2.2.4 Test Operations...................................................................... 30

7.2.2.5 Test Report Production............................................................... 30

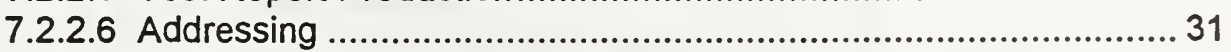

7.2.2.7 Evaluation of Conformance Testing............................................. 31

7.2.3 Treatment of Derived Products ..................................................................... 31

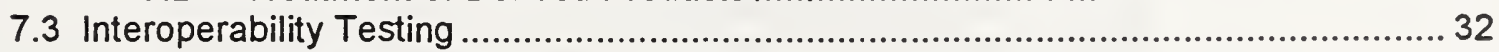

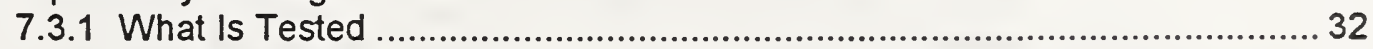

7.3.2 How Testing Is Conducted ..................................................................... 33

7.3.2.1 Requirements of an Interoperability Testing Suite........................ 33 
7.3.2.2 Requirements of an Interoperability Testing Service .................. 34

7.3.3 Treatment of Derived Implementations ....................................................... 35

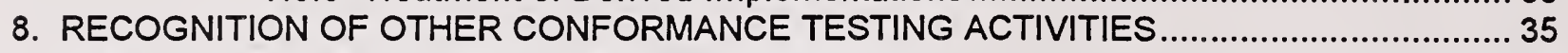

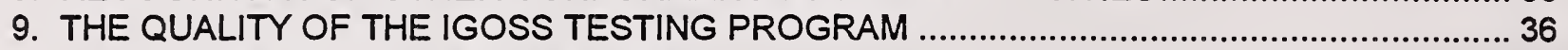

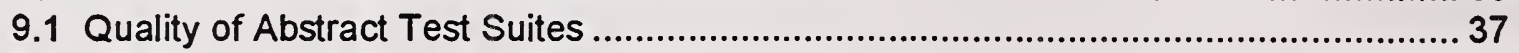

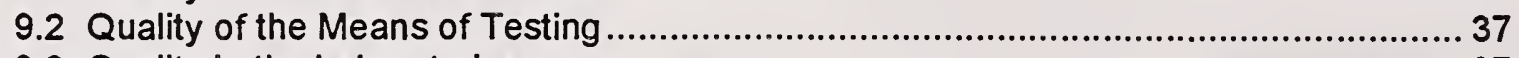

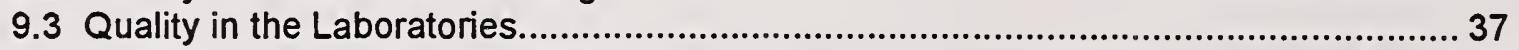

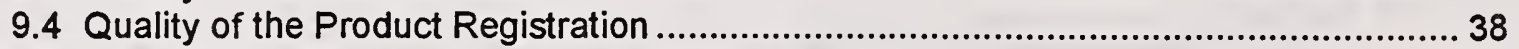

9.5 Quality in the Vendor Development Process...................................................... 38

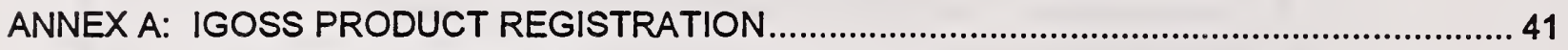

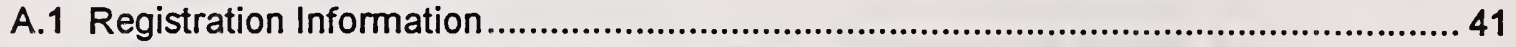

A.1.1 Summary: How to Become Registered .............................................. 41

A.1.2 Base Products ............................................................................... 42

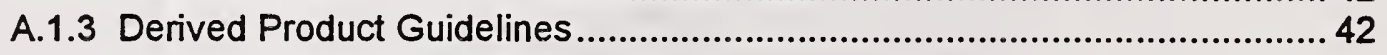

A.1.4 Derived Product Guideline Analysis.............................................. 43

A.1.5 Mutual Recognition Arrangements............................................... 47

A.1.6 Product Register Structure ............................................................... 48

A.1.7 Registration Categories .................................................................. 50

A.1.8 IGOSS Implementation Philosophy ..............................................50

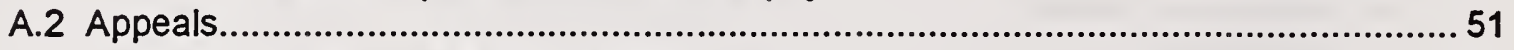

A.3 Related Conformance Testing Information ...................................................... 51

A.3.1 Test Case De-selection Procedures................................................ 52

A.3.2 The Distinction Between "Serious" and "Trivial" Errors .............................. 55

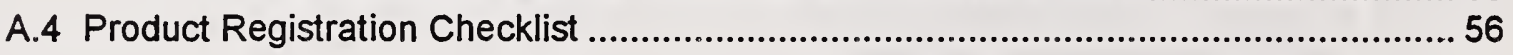

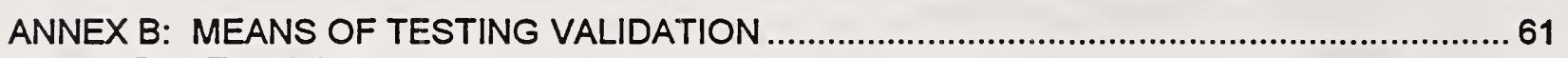

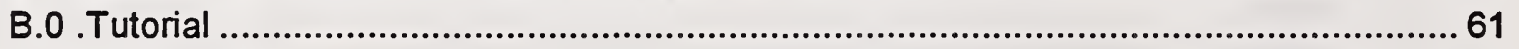

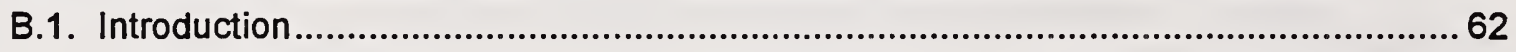

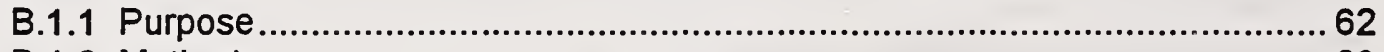

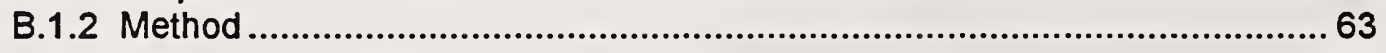

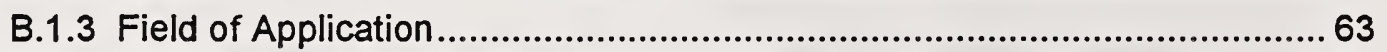

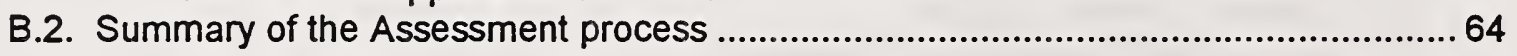

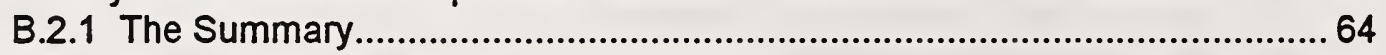

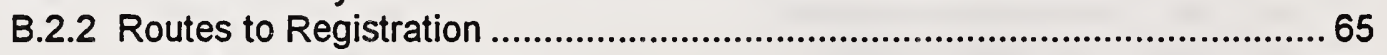

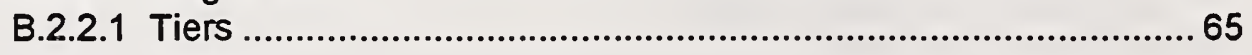

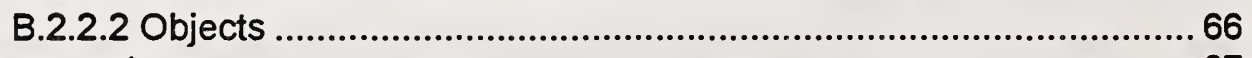

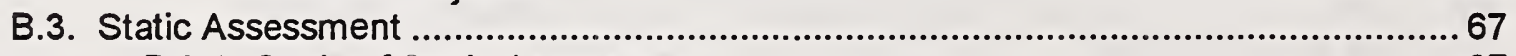

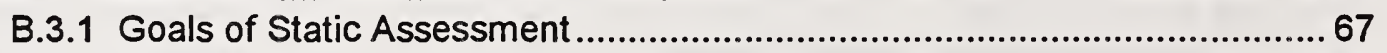

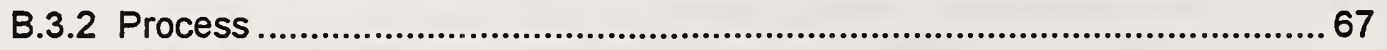

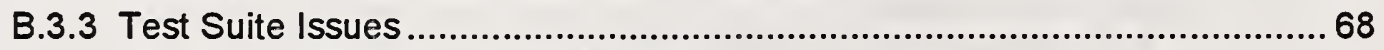

B.3.3.1 Abstract Test Suite Validity ..................................................68 68

B.3.3.2 Coverage Guidelines.........................................................68 68

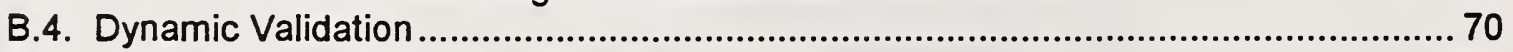

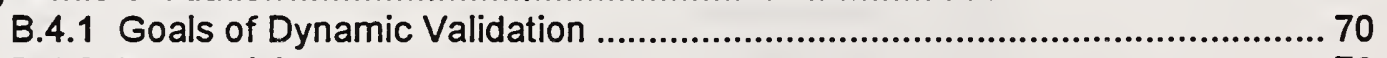

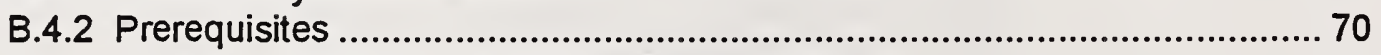

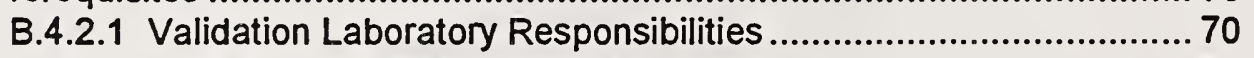

B.4.2.2 MOT Supplier Responsibilities ............................................ 70

B.4.2.3 Shared Responsibilities ....................................................... 71

B.4.3 Dynamic Assessment Procedures................................................... 72

B.4.3.1 Complete Assessment .................................................... 72

B.4.3.2 Partial Assessment ........................................................ 73

B.4.4 Assessor's Report........................................................................... 73

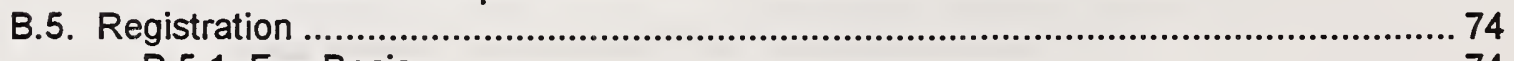

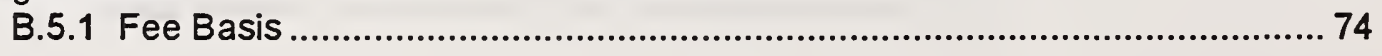


B.5.2 Categories and Criteria for Registration ............................................... 74

B.5.3 Derived Implementation Guidelines................................................... 77

B.5.4. Additional Guidance for MOT Registrations .......................................... 77

B.5.4.1 Definitions........................................................................... 78

B.5.4.2 Generic Guidance ............................................................... 79

B.5.5 Mutual Recognition Arrangements ................................................... 82

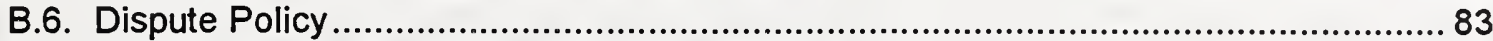

B.6.1 Challenges to Base Implementations .................................................. 83

B.6.2 Challenges to Derived Implementations .............................................. 83

B.6.3 Challenges to 'Mutual Recognition Arrangement' Implementations ............. 83

B.6.4 Abstract Test Suite Problem Resolution................................................... 84

B.6.5 Reference Implementation Problem Resolution ................................... 84

ANNEX C: HOW TO ACCESS THE REGISTERS ......................................................... 85

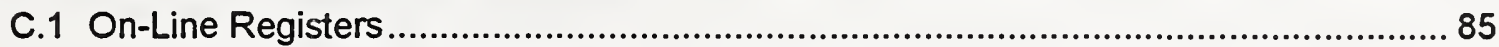

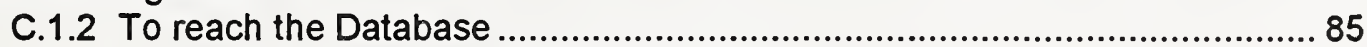

C.1.2 For server access to downloadable files: ............................................. 86

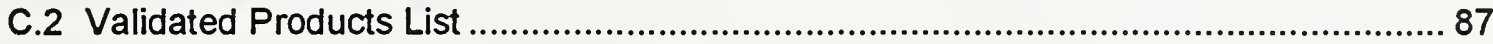

ANNEX D: ADVICE TO PROCUREMENT AUTHORITY .................................................... 89

D.1 Introduction and Statement of the Problem ........................................................ 89

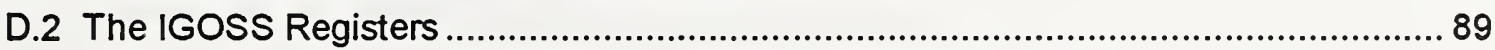

D.2.1 The Register of Conformance Tested IGOSS Products ............................ 90

D.2.2 The Register of Interoperability Testing Services..................................... 92

D.3. Procurement Strategy ......................................................................... 92

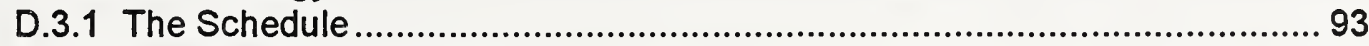

D.3.2 Specification of Options ....................................................................... 93

D.3.3 Assurance of Troubleshooting/Interoperability Support ............................ 93

D.4 Acquisition Testing Strategy...................................................................... 94

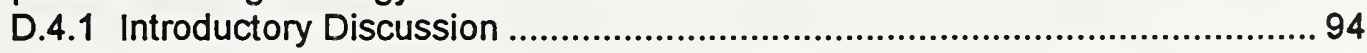

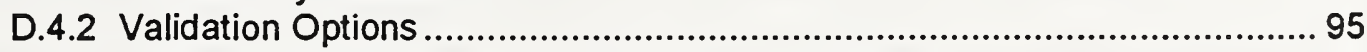

D.4.3 Adding IGOSS Capability to Existing Installations .................................... 96

D.4.4 Interoperability Testing as Extra Insurance for Product Acceptability ......... 96

D.4.5 Extra Testing Possibilities................................................................... 97

D.4.5.1 Security Features............................................................. 97

D.4.5.2 Performance Requirements .............................................. 97

D.4.6 A Note on Diagnostic Equipment .................................................. 97

D.4.7 Holes in the Testing Infrastructure ...................................................... 98

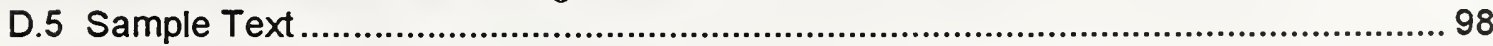

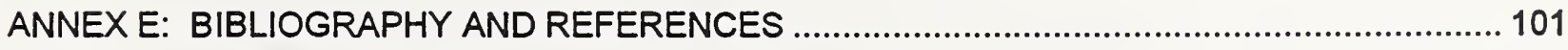




\section{OVERVIEW}

The Industry/Government Open Systems Specification (IGOSS) Partners (i.e., the U.S. Federal Government, the Canadian government, Manufacturing Automation Protocol (MAP) User Group, the Technical and Office Protocol (TOP) User Group, and the electric power industry) have developed a number of networking profiles based on Open Systems Interconnection (OSI) standards. The resulting National Institute of Standards and Technology (NIST) Special Publication 500-217 which specifies the Industry/Government Open Systems Specification (IGOSS) [NIST 15], leads to the establishment of procedures to assess which networking products conform to the IGOSS profiles and which are interoperable.

This document describes the procedures for:

- the registration and review of IGOSS abstract test suites;

- the assessment of IGOSS test tools;

- the accreditation of IGOSS testing laboratories;

- the registration of IGOSS tested products; and

- the assessment and registration of organization providing IGOSS interoperability testing.

The eventual usage of such registers is beyond the scope of this document and is expected to appear in the Acquisition Statement of each IGOSS partner.

To achieve this goal, this document, which supersedes NISTIR 4594, references other publicly accessible registers, other publications, and works conducted under the auspices of:

- the National Voluntary Laboratory Accreditation Program,

- $\quad$ the Open Systems Environments (OSE) Implementors' Workshop,

- the IGOSS Panel or its designated Agent, and

- the International Organization for Standardization (ISO).

The IGOSS Testing Framework addresses the following issues:

- IGOSS Conformance Testing: IGOSS Conformance Testing shall be conducted by accredited test laboratories using assessed and registered Means of Testing. Registered results of conformance testing will be published periodically for purposes of Industry/Government procurement. Mutual recognition of other conformance testing authorities' results will be considered using these criteria as a basis. 
- $\quad$ IGOSS Interoperability Testing: For suppliers claiming IGOSS conformance, interoperability testing against IGOSS compliant peer-entities is advised for IGOSS application and relay stacks. Criteria and procedures are identified to select and register Interoperability Testing and Registration Services.

- $\quad$ Laboratory Accreditation: Accreditation Policies and procedures are published under the auspices of the National Voluntary Laboratory Accreditation Program (NVLAP) and include: policy, procedures and criteria to determine that candidate test laboratories are qualified to conduct IGOSS product testing; procedures and criteria to determine that registered test methods are employed by candidate test laboratories. Mutual recognition of other Accreditation authorities' results will be considered using these criteria as a basis. This report does not distinguish between a conformance testing laboratory which is first party (self-testing) or third party (independent of product supplier).

- $\quad$ Abstract Test Suites: Criteria identified for test suite coverage in this report shall be applied to identify or develop, amend as necessary, and maintain a set of Abstract Test Suites for IGOSS. Abstract test suites registered by the NIST shall be used as the standard reference for the assessment of Means of Testing.

- $\quad$ Means of Testing: Assessment of means of Testing (MOT) shall be conducted by accredited test laboratories approved by the Agent of the IGOSS Panel. Registered MOTs will be published periodically. Mutual recognition of other MOT assessment authorities' results will be considered using these criteria as a basis.

Public Registers: Registers will be maintained and published periodically for the following:

1) PICS Proformae;

2) Abstract Test Suites;

3) Reference Implementations for MOT Assessment;

4) Qualified Means of Testing;

5) Accredited Laboratories;

6) Successfully Conformance Tested IGOSS Products;

7) Interoperability Test Suites; and

8) Interoperability Testing and Registration Services. 
INDUSTRYIGOVERNMENT OPEN SYSTEMS SPECIFICATION TESTING FRAMEWORK

GENERAL REQUIREMENTS FOR TESTING IGOSS 



\section{INTRODUCTION}

Acceptance of vendor products for use within Industry/Government operations is the responsibility of an Industry/Government Acquisition Authority. Normally, an Acquisition Authority develops an acceptance test plan to evaluate the functional and performance characteristics of proposed products against requirements specified within a request for proposal (RFP). When an Acquisition Authority introduces a requirement for standards compliance within an RFP, a new testing issue is created - determining compliance of proposed products with the standard. When a data communication profile, such as the Industry/Government Open Systems Specification (IGOSS) [NIST 25] is cited, an additional testing issue arises - determining interoperability between proposed products and existing products that are known to comply with the IGOSS.

The IGOSS Testing Framework is intended to provide the Acquisition Authority with as much assistance as possible to determine compliance to IGOSS and to demonstrate interoperability between vendor products purporting to comply with IGOSS. The Acquisition Authority should reserve the right to test proposed products against more stringent criteria should the need exist and should the cost be justified. In such cases, the following IGOSS test policy will provide a significant foundation.

\subsection{Background}

The Industry/Government Open Systems Specification (IGOSS) is jointly authored by the U.S. Government, the Canadian government, Manufacturing Automation Protocol (MAP) User Group, the Technical and Office Protocol (TOP) User Group, and the electric power industry. Each of these five major user organizations have previously issued their own procurement profiles to coordinate the acquisition and operation of computer networking products and services based on the international Open Systems Interconnection (OSI) standards.

The MAP specification [MISC 1] was first published by the General Motors Corporation in 1984. Other companies joined the effort to promote the use of the OSI protocols by the factory automation community. Under the leadership of General Motors, the MAP Users Group was formed which supported General Motors in the work of producing subsequent versions of the MAP specification. Around the same time, the office and engineering community recognized the importance of developing an OSI procurement specification which would accelerate the availablility of off-the-shelf computer networking products that would meet the needs of users. The first version of the TOP specification [MISC 2] was published in 1985 by the Boeing Corporation and, under Boeing's leadership, the TOP Users Group was formed in the latter part of that year. The MAP and TOP communities joined forces to coordinate their activities under common North American and World Federation of MAP/TOP Users Groups. The MAP and TOP specifications are now maintained by the World Federation of MAP/TOP Users Groups. The Corporation for Open Systems (COS) distributes both the MAP and TOP documents. The MAP and TOP organizations also jointly organized the 
Enterprise Networking Event in 1988 at which 52 vendors demonstrated that OSI products can be used to solve real business problems.

In late 1986 the National Bureau of Standards (NBS), now the National Institute of Standards and Technology (NIST) initiated development of the Government Open Systems Interconnection Profile (GOSIP). A Federal inter-agency group of experts was formed, and evolved into the GOSIP Advanced Requirements Group, that now has responsibility for promulgating each new version of the GOSIP. The NIST chairs this group and has editing responsibility for the document. At the time the first draft of GOSIP was written, the MAP and TOP specifications were nearing stability, vendor OSI implementations were being successfully demonstrated, and commercial OSI products were just entering the marketplace. The intent of GOSIP is to transmit Federal user requirements to vendors and to encourage vendors to build OSI products satisfying those requirements. The GOSIP, unlike the MAP and TOP documents, is a mandate. GOSIP mandates that Federal agencies acquire OSI products when acquiring the services provided by the OSI protocols referenced in the document. Since the GOSIP must be referenced in Federal procurement requests, where applicable, GOSIP contains only those OSI protocols which are expected to be implemented in vendor products. The MAP and TOP documents included some specifications of OSI protocols which those communities wanted the vendors to implement in the future. Accordingly, although informal coordination has existed between the MAP/TOP and Federal communities, the protocols in GOSIP have tended to be a subset of the protocols in the union of the MAP and TOP documents.

In order to promote interoperability among computer systems supplied to the electric power industry, the Electric Power Research Institute (EPRI) initiated the Utility Communications Architecture (UCA) project. The first phase of the project identified the information requirements within an electric utility. Subsequent phases identified appropriate standards for inclusion in Version 1 of the UCA specification. The UCA document, like the MAP and TOP documents, is a specification of user requirements. Twenty utility companies participated in the review of the draft document, which was then formally released to the vendor community. EPRI continues to have the responsibility for maintaining the UCA specification. OSI protocols are the foundation on which Version 1 of the UCA specification is based. The UCA authors were knowledgeable of the MAP, TOP, and GOSIP documents and recognized the importance of aligning specifications so that vendors would not be forced to build a different set of products for each new user community.

In April 1987, the Canadian federal government, announced a new policy on OSI. This policy, which applies to all Canadian government departments, endorses OSI as a Information Technology (IT) strategy in preference to any manufacturer-specific or installation-specific architecture and requires that departments and agencies state a clear preference for OSI-based products and services in their procurements. In order to assist users to migrate to OSI, work began in 1987 to develop the Canadian Open Systems Application Criteria (COSAC). This work is led and coordinated by the Treasury Board Secretariat (TBS) which is responsible for Canadian government IT 
standards and policy. COSAC comprises endorsements of the OSI based standards, OSI functional profiles, and guidance documents, all of which are published as Treasury Board Information Technology Standards. In producing COSAC, maximum alignment with other government and international specifications has also been an objective. Thus, cooperation between the five user communities to produce the IGOSS is just a formal extension to what has existed informally for some time.

This specification is the standard reference for all IGOSS organizations to use when acquiring and operating ADP systems or services and communications systems or services intended to conform to Open Systems Interconnection protocols. This specification will allow major network users in Canada and the United States to consolidate their procurement and operational requirements in a single document and is expected to be welcomed by OSI vendors because it implicitly represents significant purchasing power.

\subsubsection{Evolution of the IGOSS}

An IGOSS Panel, which consists of members from each IGOSS organization, is responsible for creating a procurement specification that meets the common requirements of the participating user groups. Since the IGOSS will be referenced in procurement requests, the document can only include functionality which vendors are in the process of implementing or have already implemented.

For this version and all subsequent versions of the IGOSS, the IGOSS Panel, after consulting with members of their respective organizations, recommends the protocols and services to be included in the common procurement specification. In making this decision, the IGOSS Panel considers the progress made in developing the standards and implementors agreements and the commitment of the vendors to develop products based on these documents. The IGOSS Panel members are then responsible for obtaining formal concurrence on the proposed content of the draft document from the organizations that they represent. Members of IGOSS organizations, as well as industry and government reviewers, will use a ninety-day public comment period as the mechanism to comment on the document. The IGOSS Panel will then modify the draft version of the IGOSS, incorporating those comments which are consistent with the objectives of the IGOSS organizations. The panel members will then obtain final approval of the document from their respective organizations before publishing the document in final form. (The IGOSS will be published as a NIST special publication in the United States and as a Treasury Board IT Standard in Canada.) This approval process will apply when each new version of the IGOSS is issued.

IGOSS will be updated by issuing new versions at appropriate intervals, tentatively every two years, to reflect the progress being made by vendors in providing OSI products with new services for government and commercial uses. A new version of IGOSS will supersede the previous version of the document. Every attempt will be made to obtain backward compatibility with the previous version of the document. Every new version of IGOSS will specify the architecture and protocols that were 
included in each of the previous versions so that the protocols added to each version can easily be determined.

\subsubsection{Scope of IGOSS}

In an increasingly complex world, the need to exchange information has become an ever more important factor in conducting business. Until recently, computer networking technology has not kept pace with this need to communicate. Even now, many users have "islands" of computer systems built by different vendors, or even by the same vendor, that cannot exchange information. The IGOSS indicates that, in response to this, the vendor community has developed a nonproprietary solution for this requirement to exchange information. The solution uses OSI protocols, allowing computer systems built by different vendors to exchange data. These OSI protocols give users access to standardized applications which can operate over diverse reliably interconnected subnetworks. The IGOSS lists the protocol specifications and provides procurement alternatives.

IGOSS significantly expands the scope of user services provided by OSI applications. The IGOSS electronic mail service uses the OSI standard for Message Handling Systems (MHS). There are two major MHS components: the Message Transfer System (MTS) and the cooperating user agents. IGOSS specifies two types of user agents for which International Standardized Profiles are currently under develpment. Additional user agent (UA) types may be specified by procurers. The two internationally standardized User Agents are the Interpersonal Messaging User Agent, used to send a personal message from an originator to one or more recipients, and the Electronic Data Interchange User Agent, used to send and receive business related tranactions using standard transaction sets. File transfer services are provided by the File Transfer, Access, and Management (FTAM) application. A remote terminal access capability is provided by the Virtual Terminal (VT) application. The Directory Services application provides access to a distributed directory on behalf of human users or OSI applications such as MHS or FTAM. The Remote Database Access (RDA) application allows the interconnection of database applications resident in heterogeneous environments. The Transaction Processing (TP) application provides for reliable support of distributed, interdependent transactions. The X-Windows application allows a user to gain access to multiple computer applications by dividing a screen into multiple sections, each section responding independently to input from a keyboard or a pointing device or both. The Manufacturing Messaging Specification (MMS) application allows objects related to a process control environment to be accessed and manipulated across a network. The Information Retrieval (IR) application provides information search and retrieval by converting queries constructed in a local query language into a common representation. All of these applications use lower layer OSI protocols to guarantee that end systems attached to subnetwork technologies [e.g., X.25 Wide Area Network (WAN), Local Area Network (LAN), Integrated Services Digital Network (ISDN), Frame Relay] can interoperate. 


\subsubsection{Relationship of the IGOSS to Existing Profile Documents}

The IGOSS is a collaborative effort of organizations that have previously published the Canadian Open System Application Criteria, the Manufacturing Automation Protocol specification, the Technical and Office Protocol specification, the United States Government Open Systems Interconnection Profile and the Utility Communications Architecture documents. These documents will continue to be published by the responsible IGOSS organizations, but now they will primarily refer to the IGOSS to specify the OSI procurement requirements for each organization. The documents will also contain specifications for any protocol required by the organization, but not agreed to in common, and an applicability statement which indicates how the IGOSS must or should be used. The Federal Government will continue to promulgate the Government Open Systems Interconnection Profile as a Federal Information Processing Standard (FIPS). In Canada, the Canadian Open Systems Application Criteria will continue to be developed as a Treasury Board IT Standard (TBITS).

\subsubsection{Applicability}

The IGOSS specifies a set of OSI protocols for computer networking that is intended for acquisition and use by IGOSS organizations. Each IGOSS organization will specify the applicability of IGOSS to its own members. The detailed statements of applicability will appear in supplementary profile documents, issued by each IGOSS organization.

\subsubsection{IGOSS Functionality}

Version 2 of GOSIP was the base document used to prepare the IGOSS. All GOSIP Version 2 protocols are included in the IGOSS. The functionality added to the base document to form Version 1 of the IGOSS is as follows:

1. Message Handling Systems (CCITT 1988 Recommendation);

2. Electronic Data Interchange (EDI) User Agent;

3. File Transfer, Access, and Management (FTAM) - (Phase 3);

4. Virtual Terminal Service (S-mode Paged and X.3 profiles);

5. Directory Services;

6. Remote Database Access;

7. Transaction Processing;

8. Manufacturing Message Specification;

9. X-Windows over OSI;

10. Information Retrieval;

11. Fiber Distributed Data Interface (FDDI);

12. Frame Relay;

13. Point to Point Protocol (PPP);

14. Intermediate System-Intermediate System routing (IS-IS) protocol;

15. Inter-Domain Routing Protocol (IDRP);

16. Network Management protocols; and

17. Connectionless Upper Layer services.

18. Minimal OSI Upper Layer Services 


\subsubsection{Sources of Protocols Specification}

\subsubsection{Primary Source}

\section{Relationship of the IGOSS Specifications to Workshop Agreements}

The primary source of protocol specifications in the IGOSS is the Stable Implementation Agreements for Open Systems Interconnection Protocols [NIST 1], hereafter referred to as the Workshop Agreements. By primary source, it is meant that where the IGOSS uses a given protocol, it cites that protocol by reference to the Workshop Agreements. The primary source is used in all instances where the protocol of interest has been specified in the Workshop Agreements. Section 4 of this specification augments those agreements when necessary to provide the functionality required by IGOSS organizations.

The primary source document was created and is maintained by the Open Systems Environment (OSE) Implementors Workshop (OIW). The Workshop Agreements provide implementation specifications that are derived from service and protocol standards issued by the International Organization for Standardization (ISO) and the Consultative Committee for International Telegraphy and Telephony (CCITT). A copy of the Workshop Agreements is essential to thoroughly understand the material in this document.

A new version of the Workshop Agreements is created each year, following the December OSE Implementor's Workshop meeting, if a sufficient amount of new functionality has accumulated since the previous version was issued. It is the intent of the Workshop that new versions of the Workshop Agreements be backwardly compatible with previous versions. Replacement pages applying to the latest version of the Workshop Agreements may be published at regular intervals during the year. These replacement pages may contain errata to the original stable agreements that are approved by the Workshop plenary. The latest replacement pages are distributed to all workshop attendees and are available through several sources. (See NIST Reference 1 for ordering information.)

Each new version of the IGOSS will reference the latest appropriate version of the Workshop Agreements as the base document. These agreements, although stable, can be modified by errata which correct technical and editorial mistakes or by changes which are required to align with evolving international standards or agreements developed in other regional workshops. These changes to the Workshop Agreements are stabilized each December and become effective as an implementation requirement the following December (e.g., the stabilized Workshop Agreement for December, 1993 becomes effective as an implementation requirement as of December, 1994). This becomes effective with the December, 1993 Workshop Agreements.

Each version of the IGOSS is issued in draft form for public comment before it is issued 
in final form. Final editions of the IGOSS will reference only the Stable Workshop Agreements.

\section{Relationship of the IGOSS Specifications to International Standardized Profiles}

International Standardized Profiles (ISPS) are functional profiles which are approved for publication by the ISO/IEC JTC1 Special Group on Functional Standards (SGFS) under SGFS procedures. These functional profiles should be technically harmonized at the regional workshop level before submission to the SGFS.

It is a goal that the IGOSS technical specifications reference ISPs, when possible. The linkage by which this will occur will be the Workshop Agreements. For each protocol, the appropriate Special Interest Groups will determine if and when it is appropriate to replace the existing Workshop Agreement text with a reference to an ISP.

\subsubsection{Secondary Sources}

The IGOSS must be complete in that open systems procured in accordance with it must interoperate and must provide service generally useful for government and commercial computer networking applications. The Workshop Agreements continue to evolve, but remain incomplete. (The appendices of the IGOSS cite needed work.) Thus, where the Workshop Agreements are not complete, the IGOSS may augment protocol and service specifications from the following sources.
- International Standards and Recommendations
- Draft International Standards
- Institute of Electrical and Electronics Engineers (IEEE) Standards
o Working Implementation Agreements from the OIW

Since this profile is one of open systems, the secondary sources include specifications that are international standards or are advancing to become international standards. They are included in the IGOSS, where needed, to help satisfy the criterion of utility. Note that secondary sources exclude protocols, however mature, that are not a part of the international standards process.

\subsubsection{Tertiary Sources}

Even the secondary sources named above may not provide a complete and useful networking system today. It may be necessary for the IGOSS to augment protocol and service specifications from the following sources.
- National Standards
- Government Standards
- Military Standards
- Other publicly available specifications

The use of specifications from other than the primary and secondary sources is 
undesirable. It is expressly intended that these omissions from standards work be brought to the attention of the international standards bodies so that acceptable international standards may be developed as rapidly as possible. The IGOSS Panel will replace all tertiary source protocols in the IGOSS with suitable primary and secondary sources, as soon as they are available.

\subsubsection{IGOSS Errata}

All errata to the IGOSS will be subject to the same public review process that exists for the IGOSS document. The errata may take effect at any time after the public review period if approved by the IGOSS Panel. Since each new version of the IGOSS will supersede the previous version, errata to previous versions will be published in subsequent versions.

\subsection{Purpose of IGOSS Testing Framework}

The purpose of this document is to provide the framework for uniform Industry/Government-wide procurement of IGOSS conformant products. As such, this document is intended to inform government agencies, industry, standards development bodies, and other interested organizations of the IGOSS policy with regard to conformance testing and interoperability testing of IGOSS products.

The objectives of the IGOSS Testing Framework are:

- To reduce the overall information systems costs by making it easier and less expensive to maintain information technology applications and to transfer these applications among different information systems, including replacement systems;

To protect the technical assets and staff time of the Industry/Government by insuring to the extent possible that purchased products comply with IGOSS;

To identify test methods and competent test laboratories for assisting Industry/Government in the procurement of industry supplied IGOSS products;

To increase the likelihood of interoperability of IGOSS conformant products.

Further detailed solutions to the guidelines in this document are given in a companion handbook:

The NVLAP Program Handbook: Operational Requirements of the Laboratory Accreditation Program for IGOSS Conformance Testing [NVLAP 1] and NIST Handbook 150, National Voluntary Laboratory Accreditation Program. Procedures and General Requirements [NVLAP 2] provides the administrative procedures for NVLAP accreditation. 
The SCC Handbook, Information Technology and Telecommunications (IT\&T) Laboratory Accreditation Program [SCC 5] provides the administrative procedures for SCC Accreditation.

\subsection{Scope}

This document provides detailed advisory provisions with respect to conformance and interoperability testing given in "Industry/Government Open Systems Specification (IGOSS) Version 1.0". This document defines policy and procedures related to conformance testing and interoperability testing for IGOSS. Other types of testing such as performance, acceptance, and quality testing, are not addressed.

In determining testing requirements for IGOSS, a number of areas are considered: Industry/Government testing needs, test method technology, standard specifications, alternative testing sources (third-party testing, Industry/Government testing, self-testing, etc.), and existing accreditation and certification systems.

The policy and procedures for conformance testing and for interoperability testing defined herein apply whenever IGOSS standards are required to support Industry/Government objectives for information systems.

The report is addressed to:

1) Procurement Authorities intending to procure OSI products;

2) Suppliers of OSI products wishing to market to the IGOSS Partners;

3) Suppliers of OSI test services seeking accreditation as a test laboratory;

4) Developers of the means of testing OSI products wishing to supply to accredited laboratories.

5) Suppliers of OSI interoperability testing and registration services seeking recognition by the IGOSS Partners.

The program of registration will be administered by the IGOSS Panel, or its appointed agent. In the text that follows the acronym "Agent of the IGOSS Panel" is used to mean "the Agent of the IGOSS Panel or any organization supporting the Agent of the IGOSS Panel".

\subsection{Overview of Testing}

This document is concerned with conformance and interoperability testing from the point of view of both their conduct and the evaluation of their methods. To eliminate 
confusion over which role is being addressed this document draws the distinction between the terms assessment and accreditation on the one hand, and testing on the other.

Testing means using tools, facilities and procedures to establish that implementations of IGOSS related products are conformant and/or interoperable.

Means of Testing Assessment is the process of determining that testing tools are fit for their declared purpose and of producing a MOT validation/calibration report.

Accreditation is the administrative act of recognizing that: 1) a test laboratory is qualified to conduct protocol testing after having specifically met both the quality and technical criteria, and 2) the means of testing employed by a test laboratory meets specific technical criteria.

Registration is the administrative act of recognizing that the tested products meet specified criteria by registering the results after successful testing has been conducted. Registration is used in lieu of issuing certificates of conformity.

Within the International Organization for Standardization (ISO) conformance testing methodology has developed and is the subject of a separate standard (IS 9646 OSI Conformance Testing Methodology and Framework) [ISO 113]. Its purpose is to define standardized methods which may be used for conformance testing and to define relationships between: 1) parties supplying the means of testing OSI protocols and test laboratories, and 2) test laboratories and their clients, and the information exchanged between them. Conformance testing concentrates on determining whether an implementation of a protocol conforms to both static and dynamic requirements specified in a protocol standard.

Current conformance testing technology provides for tools which separate the testing concerns of any 7-layer OSI stack into three functional groups:

- Upper Layers:

Session, Presentation, Application

- Intermediate Layers: Network, Transport

- Lower Layers:

Physical, Link

Higher layer protocols are tested, and operated, over a stack of supporting protocols. IS 9646 prescribes testing of the lower layer protocols prior to testing the protocols which they support. One reason for this arrangement is that direct access to a layer service affords the greatest possible capability of controlling and observing events within that layer. Another reason is that the development of the means of testing followed the development of protocol stack implementations, and the means of testing for lower-layer protocols were available earliest. An advantage afforded by this approach is that the Protocol Conformance Test Report (PCTR) can be used in support of incremental testing, specifically so that full regression testing is not needed in testing 
a larger stack which builds on the already tested functionality.

Full-stack testing is also an alternative, although no 7-layer means of testing is currently in existence. Full stack methods require that each protocol in the stack be tested by embedded methods (except the Application protocols which are tested by single-layer methods). This procedure is repeated for every new Application stack, since each new service user may exercise paths within a lower layer protocol which are not explored by a different service user in another full stack.

More recently interoperability testing has been identified as a necessary complementary step in demonstrating interworking of OSI implementations. Whereas the failures in conformance testing are likely to be software errors, interworking problems seem more likely to include problems of parameter range selection, plus attempts to use incompatible stacks, attempts to use optional functions not implemented, and failures to implement mandatory functions. The methodology and test suites employed are subject to the criteria given in clause 6.3. Since it is necessary to assess the interoperability of systems, this report identifies multi-vendor bilateral interoperability testing.

\subsection{Definitions}

Abstract Test Case: A complete and independent specification of the actions required to achieve a specific test purpose (or a specified combination of test purposes), defined at the level of abstraction of a particular abstract test method. It may include a preamble and postamble to ensure starting and ending in a stable state (i.e. an identifiable stable state of the System Under Test which can be easily reached and maintained, such as the 'idle' state or the 'data transfer' state). This specification may involve one or more consecutive or concurrent connections.

Abstract Test Method: The description of how an Implementation Under Test is to be tested, given at an appropriate level of abstraction to make the description independent of any particular implementation of testing tools, but with enough detail to enable tests to be specified for this test method.

Acceptance Testing: Formal testing conducted to determine whether or not a system satisfies its acceptance criteria and to enable the customer to determine whether to accept the system. Formal testing may include the planning and execution of several kinds of tests (e. g., functional, volume, performance tests) to demonstrate that the implementation satisfies the customer requirements.

Accreditation Body: An impartial body, governmental or non governmental, possessing the necessary competence and reliability to operate or accredit operation of an accreditation system, and in which the interests of all parties concerned with the function of the system are represented. 
Basic Interconnection Tests: Limited tests of an Implementation Under Test (IUT) to determine whether or not there is sufficient conformance to the relevant protocol(s) for interconnection to be possible, without trying to perform thorough testing.

Behavior Tests: Tests to determine the extent to which the dynamic conformance requirements are met by the IUT.

Capability Tests: Tests to determine the capabilities of an IUT. (Note, this involves checking all mandatory capabilities and those optional ones that are stated in the Protocol Implementation Conformance Statement (PICS) as supported, but not checking those optional ones which are stated in the PICS as not supported by the IUT.)

Conformance: In the context of OSI a real system is said to exhibit conformance if it complies with the requirements of applicable OSI standards in its communication with other real systems.

Conformance Testing: Testing the extent to which an IUT is a conforming implementation.

Coordinated Test Method: An external test method for which a standardized test management protocol is defined as the test coordination procedures, enabling the control and observation to be specified solely in terms of the lower tester activity, including the control and observation of test management PDUs.

Distributed Test Method: An external test method in which there is a PCO at the layer boundary at the top of the IUT.

Dynamic Conformance Requirements: All those requirements and options which determine what observable behavior is permitted by the relevant OSI standards in instances of communication.

Dynamic Interoperability Requirements: All those requirements and options which determine what observable behavior is permitted between peer open systems by compatible standardized profiles of OSI standards, in instances of communication.

Embedded Testing: Testing the behavior of a single layer within a multi-layer IUT without accessing the layer boundaries for that layer within the IUT. (This is contrasted with 'exposed' testing in which the N-service PCO of the IUT is accessible for testing.)

Equivalent Configuration: Any configuration for which conformance is achievable using the same registered test method version used in conformance testing of an implementation under test. 
IGOSS Product: A product which implements one or more of the data communications protocols identified in IGOSS and meets the requirements specified herein.

Implementation Under Test (IUT): An implementation of one or more OSI protocols in an adjacent user/provider relationship, being that part of a real open system which is to be studied by testing.

Interconnection: Establishment of communication between peer protocol entities over a physical medium or an OSI layer service.

Interoperability: The capability of one entity to collaborate with one or more other peer entities to render a particular service to their respective users.

Interoperability Test Case: An interoperability test case describes the actions to be carried out and the rules for verdict assignment in order to test for interoperability using one single well defined interoperability test purpose.

Interoperability Test Method: An interoperability test method describes the set of actions and associated procedures that guides a real open system through interoperability evaluation, with one or more real peer open systems, in order to evaluate the degree of interoperability achieved by the systems involved.

Interoperability Test Purpose: A prose description of a narrowly defined objective of interoperability testing, focusing on a single interoperability requirement.

Interoperability Test Report: An interoperability test report contains the record of all the test results as described in the test records, together with a description of the environment in which the tests have been executed.

Interoperability Testing: A process where two or more products execute the interoperability test suite according to an interoperability test method.

Means of Testing: The realization of an abstract test method as defined in the OSI Conformance Testing Methodology and Framework. This realization includes the test system, executable test suite, testing support tools (hardware and software) and documentation (including technical test procedures).

Multi-Layer Testing: Testing the behavior of a multi-layer IUT as a whole, rather than testing it layer by layer (in contrast to Single-Layer Testing).

National Voluntary Laboratory Accreditation Program (NVLAP) and Standards Council of Canada (SCC): Voluntary systems for accrediting laboratories found competent to perform specific testing operations. NVLAP is part of the National Institute of Standards and Technology Office of Associate Director for Industry and 
Standards. NVLAP and SCC do not confer product or test data certification.

Out-of-Band Coordination: A separate communications path used for test coordination procedures which may be realized from a lower-layer service or alternative physical media.

Product Interoperability Test Report: This is a document written at the end of the interoperability testing process, giving the details of the testing carried out for a specific interoperability test suite.

Proficiency Testing: Determination of laboratory testing performance by means of comparison of tests on the same or similar items by two or more laboratories in accordance with predetermined conditions.

Protocol Conformance Test Report (PCTR): A document written at the end of the conformance assessment process, giving the details of the testing carried out for a particular protocol. It includes the identification of the abstract test cases (if these exist) for which corresponding executable test cases were run. It also includes the test purpose(s) and verdict for each test case.

Protocol Implementation Conformance Statement (PICS): A statement made by the supplier of an OSI implementation, or system, stating which capabilities and options have been implemented, for a given OSI protocol.

Protocol Implementation eXtra Information for Testing (PIXIT):

A statement made by a supplier or implementor of an IUT which contains or references all of the information (in addition to that given in the PICS) related to the IUT and its testing environment, which will enable the test laboratory to run an appropriate test suite against the IUT.

Remote Test Method: An external test method in which there is neither a PCO above the IUT nor a standardized test management protocol; some requirements for test coordination procedures may be implied or informally expressed in the abstract test suite but no assumption is made regarding their feasibility or realization.

Single-Layer Testing: Testing the behavior of one layer-protocol from a multi-layer IUT.

Static Conformance Requirements: Constraints which are specified in OSI standards to facilitate interworking by defining the requirements for the capabilities of an implementation.

Static Interoperability Requirements: For potentially interoperable peers these include: 
- Compatible static conformance requirements;

- Both systems successfully conformance tested;

- Peers are configured to enable interconnection.

System Conformance Test Report (SCTR): A document written at the end of the conformance assessment process, giving the overall summary of the conformance of the system to the set of protocols for which conformance testing was carried out.

System Under Test (SUT): The real open system in which the IUT resides.

Test System Environment Specification: This is a statement made by a supplier or an implementor of an OSI product which contains or references all of the information (in addition to that given in the PICS) related to the implementation and its environment, which will enable the test parties to execute an appropriate test suite against their implementations.

Verdict: A statement of "Pass", "Fail", or "Inconclusive", specified in the abstract test suite concerning conformance of an IUT with respect to a test case that has been executed.

\subsection{Abbreviations}

$\begin{array}{ll}\text { ATS } & \text { Abstract Test Suite } \\ \text { CCITT } & \text { International Telephone and Telegraph Consultative Committee } \\ \text { CEN } & \text { European Committee for Standardization } \\ \text { CENELEC } \quad \text { European Committee for Electrotechnical Standardization } \\ \text { COS } & \text { Corporation for Open Systems } \\ \text { COSAC } & \text { Canadian Open Systems Application Criteria } \\ \text { CSL } & \text { Computer Systems Laboratory } \\ \text { ECITC } & \text { European Committee for Information Technology Certification } \\ \text { EPRI } & \text { Electric Power Research Institute } \\ \text { ETCOM } & \text { European Testing for Certification for Office and Manufacturing } \\ \text { ETS } & \text { Executable Test Suite } \\ \text { EWOS } & \text { European Workshop for Open Systems } \\ \text { FIPS } & \text { Federal Information Processing Standard } \\ \text { GOSIP } & \text { Government Open Systems Interconnection Profile } \\ \text { IEC } & \text { International Electronical Commission } \\ \text { IGOSS } & \text { Industry/Government Open Systems Specification } \\ \text { INTAP } & \text { Interoperability Technology Association for Information Processing } \\ \text { IPRL } & \text { ISPICS Requirement List }\end{array}$


IS International Standard

ISO International Standards Organization

ISP International Standard Profile

ISPICS International Standard Profile Implementation Conformance Statement

IT Information Technology

IT\&T Information Technology and Telecommunications

ITU International Telecommunication Union

ITU-TS International Telecommunication Union - Telecommunication

Standardization Sector - new name for CCITT

IUT Implementation Under Test

JITC Joint Interoperability Test Center

MAP Manufacturing Automation Protocol

NIST National Institute of Standards and Technology

NVLAP National Voluntary Laboratory Accreditation Program

OIW OSE Implementor's Workshop

OSE Open System Environment

OSI Open Systems Interconnection

OSTC Open Systems Testing Consortium

PCO Point of Control and Observation

PCTR Protocol Conformance Test Report

PDU Protocol Data Unit

PICS Protocol Implementation Conformance Statement

PIXIT Protocol Implementation eXtra Information for Testing

SCC Standards Council of Canada

SCTR System Conformance Test Report

SIA Stable Implementor's Agreements

SUT System Under Test

TOP Technical and Office Protocol

\section{ORGANIZATIONAL MODEL}

Conformance and interoperability testing for IGOSS will be accomplished in accordance with the organizational model described in this Clause. This organizational model consists of a Program Authority, Program Sponsor, Accreditation Authority, the NIST OSE Implementors' Workshop, test laboratories and their clients. Each member of this model shares responsibilities for assuring conformance of products to IGOSS. Under the rules and procedures established by the IGOSS Panel, this model will enable a client to have his product tested by any NVLAP or SCC accredited test laboratory; and the test results produced by that laboratory accepted by IGOSS Panel's Agent as the basis for registration as a Conformance Tested IGOSS Product. The Product and Means of Testing registration policy and procedures employed in this organizational 
model are described in Annexes $A$ and $B$.

Full implementation or use of all parts of the organizational model depends on the complexity of the standard(s) and the conformance testing methods applied for the standard(s). In circumstances deemed necessary by the Executive Director of the IGOSS Panel, this document and registers identified by this document may designate alternate or supplementary procedures, means of testing, and abstract test suites for testing IGOSS products.

\begin{tabular}{|c|c|c|}
\hline $\begin{array}{l}\text { Clause or } \\
\text { Reference }\end{array}$ & Role & Responsible Party \\
\hline 2.1 & Program Authority & $\begin{array}{l}\text { Executive Director } \\
\text { of the IGOSS Panel }\end{array}$ \\
\hline 2.1 & Program Sponsor & IGOSS Panel \\
\hline 2.1 & Program Operator & $\begin{array}{c}\text { Agent of the IGOSS Panel } \\
\text { NIST/CSL }\end{array}$ \\
\hline 2.2 & $\begin{array}{c}\text { Test Laboratory Accreditation } \\
\text { Authority }\end{array}$ & $\begin{array}{l}\text { NVLAP or } \\
\text { SCC }\end{array}$ \\
\hline 3. & Laboratory Accreditation Procedures & $\begin{array}{l}\text { Agent of the IGOSS Panel- } \\
\text { NIST/CSL, NVLAP and SCC }\end{array}$ \\
\hline 4 & Means of Testing Assessment Authority & Agent of the IGOSS Panel \\
\hline $\begin{array}{c}4 \\
\text { Annex B } \\
\end{array}$ & Means of Testing Assessment Procedures & $\begin{array}{c}\text { Agent of the } \\
\text { IGOSS Panel - NIST/CSL }\end{array}$ \\
\hline 4. & PICS Proforma & $\begin{array}{l}\text { OSE Implementor's } \\
\text { Workshop }\end{array}$ \\
\hline 4.1 & Abstract Test Suite Review and Acceptance & $\begin{array}{l}\text { OSE Implementor's } \\
\text { Workshop }\end{array}$ \\
\hline 2.3 & Provision of Means of Testing & Test Tool Suppliers \\
\hline 2.4 & Conformance Test Services & $\begin{array}{l}\text { Accredited Conformance } \\
\text { Testing Laboratory }\end{array}$ \\
\hline 7.3 & $\begin{array}{l}\text { Interoperability Test Suite, Review } \\
\text { and Acceptance }\end{array}$ & $\begin{array}{c}\text { OSE Implementor's } \\
\text { Workshop }\end{array}$ \\
\hline 7.3 & Multi-Vendor Interoperability Testing & $\begin{array}{c}\text { OSI Product } \\
\text { Suppliers \& Users }\end{array}$ \\
\hline 5. & PICS Proforma Registration & $\begin{array}{c}\text { Agent of the IGOSS Panel } \\
\text { NIST/CSL }\end{array}$ \\
\hline 5. & Abstract Test Suite Registration & $\begin{array}{c}\text { Agent of the IGOSS Panel } \\
\text { NIST/CSL }\end{array}$ \\
\hline 5. & Interoperability Test Suite Registration & $\begin{array}{c}\text { Agent of the IGOSS Panel } \\
\text { NIST/CSL }\end{array}$ \\
\hline 5. & Accredited Test Laboratory Registration & $\begin{array}{c}\text { Agent of the IGOSS Panel } \\
\text { NIST/CSL }\end{array}$ \\
\hline $\begin{array}{c}\text { 4., 5., } \\
\text { Annex B }\end{array}$ & Means of Testing Registration & $\begin{array}{c}\text { Agent of the IGOSS Panel } \\
\text { NIST/CSL }\end{array}$ \\
\hline $\begin{array}{c}5 . \\
\text { Annex A }\end{array}$ & Conformance Tested Product Registration & $\begin{array}{c}\text { Agent of the IGOSS Panel } \\
\text { NIST/CSL }\end{array}$ \\
\hline 5. & Interoperability Tested Product Registration & $\begin{array}{c}\text { Interoperability } \\
\text { Registration Authority }\end{array}$ \\
\hline Annex D & Procurement of IGOSS Products & $\begin{array}{l}\text { Acquisition } \\
\text { Authority }\end{array}$ \\
\hline
\end{tabular}

\section{Table 1: IGOSS Testing: Organizational Responsibilities}

Table 1 provides a cross-reference of the parties involved in the model, together with 
their respective roles. The "Clause or Reference" column provides a cross-reference of each role with the respective clause of this report in which such role is described.

\subsection{Program Sponsor/Authority/Agent}

The IGOSS Panel is the Program Sponsor for the IGOSS conformance testing program. The IGOSS Panel provides the overall direction for organizing, managing, directing, and administering the IGOSS Testing Program. The Executive Director serves at the pleasure of the IGOSS Panel under the terms of the Charter of the IGOSS Panel.

The IGOSS Panel, under the direction of the Executive Director of the IGOSS Panel, has the authority to:

1) Establish and maintain the IGOSS conformance testing program policies and procedures;

2) Register the test methods used in determining conformance of products to IGOSS;

3) Develop and maintain the procedures to be followed by clients of accredited test laboratories in order to attain product registration;

4) Issue a statement of Registration based on the results of a test report;

5) Establish the accreditation criteria for OSI test laboratories;

6) Maintain and periodically publish a register of products that have passed conformance testing, and a register of products that have passed interoperability testing.

7) Coordinate with other assessment, accreditation and certification authorities for the purpose of harmonizing methods and making provisions for mutual recognition of conformance testing results;

8) Evaluate and resolve disputes on all matters concerning conformance testing for IGOSS;

9) Periodically assess the need for a conformance testing program, maintain test method assessment and test laboratory accreditation programs for IGOSS;

10) Maintain and publish a register of accredited test laboratories to perform IGOSS conformance testing;

11) Maintain and publish a register of accredited test laboratories recognized by the IGOSS Panel CSL to perform IGOSS Means of Testing Validation; 
12) Establish the fees or rates for the IGOSS Panel's Agent provided products and services; and

13) Announce in the Federal Register and/or the Commerce Business Daily the availability of an assessment service.

NIST/CSL is the permanent Agent of the IGOSS Panel. NIST/CSL is responsible for defining procedures and conducting the program of registration and assessments. Since the IGOSS Testing program is built from the U.S. GOSIP Testing Program, NIST/CSL will continue to use the services of the Joint Interoperability Test Center (JITC). In the remaining part of this document, the term "Agent of the IGOSS Panel" is used to identify NIST/CSL and/or JITC.

\subsection{IGOSS Accreditation Authority}

NVLAP and SCC are the Accreditation Authorities for IGOSS testing. The role of NVLAP and SCC is to assess and accredit testing laboratories, using the laboratory accreditation procedures provided for OSI Testing [NVLAP 1-2] or [SCC.1-6]

\subsection{Suppliers of the Means of Testing}

Suppliers undertake to develop and supply to test laboratories the means of testing. Suppliers may be commercial, governmental, educational, or foreign-based organizations.

The responsibilities of a supplier are to:

1) Develop the means of testing for IGOSS protocols in accordance with the criteria specified in this report;

2) Undertake to maintain the means of testing to reflect changes in the published standards and workshop agreements;

3) Obtain and maintain assessment and registration of their product(s);

4) Pay all relevant fees.

\subsection{Conformance Test Laboratories}

Test laboratories perform conformance testing in accordance with IGOSS Panel approved procedures. Test laboratories may be commercial laboratories (third-party), vendor laboratories (first-party), U.S or foreign-based laboratories.

Only test laboratories accredited under an IGOSS Panel approved laboratory 
accreditation program, or test laboratories established as a result of mutual recognition arrangements with NVLAP shall be recognized by IGOSS Panel to do IGOSS conformance testing.

The responsibilities of a test laboratory are to:

1) Obtain and maintain laboratory accreditation as appropriate;

2) Conduct conformance testing in accordance with the IGOSS Panel prescribed procedures;

3) Prepare SCTR and PCTRs in accordance with the IGOSS Panel prescribed procedures as a result of the testing performed;

4) Participate in proficiency testing as required;

5) Pay all relevant fees;

6) Participate in training sessions or meetings as required by the IGOSS Panel to remain up-to-date on changes to the conformance testing procedures;

7) Provide feedback to the IGOSS Panel on problems and improvements relating to the conformance testing procedures;

\subsection{Clients}

Clients are responsible for submitting requests for product conformance testing to an accredited test laboratory in accordance with testing laboratory prescribed procedures. The responsibilities of a client include:

1) Provide complete and accurate information to the test laboratory for the performance of the requested conformance testing;

2) Unless otherwise agreed to by the test laboratory, provide the test facilities and materials necessary for testing;

3) Provide a product conforming with IGOSS;

4) Provide copies of the PCTR, SCTR, and all other required documentation to the registration authority, after successful conformance testing by an accredited test laboratory, for the purpose of registration.

\subsection{Criteria for Registration of a Conformant IGOSS Product}

1) Submit to Agent of the IGOSS Panel ${ }^{\star}$ a PICS and PIXIT for a product claiming to conform to IGOSS specifications; 
2) Provide Protocol Conformance Test Report(s) and System Conformance Test Report for the product;

3) These reports are to be produced after successful conformance testing by an accredited test laboratory using a registered means of testing, including registered abstract test suites.

4) Pay the appropriate registration fees.

Annex A presents comprehensive information for IGOSS Product Registration.

\section{ACCREDITATION}

The Agent of the IGOSS Panel ${ }^{*}$ will carry out its responsibilities for conformance testing through test laboratories judged to be competent to objectively perform the necessary tests. Laboratory accreditation serves as a basis for determining laboratory competence. The purpose is to insure that testing facilities are available for obtaining an unbiased assessment of products regarding IGOSS conformance.

To ensure that test laboratories are using tools which are capable of performing accurate and adequate assessments, the IGOSS Panel defines in clause 4 the requirements upon each party involved.

Wherever appropriate for a given IGOSS protocol, the IGOSS Panel will draw upon the NVLAP and SCC as the basis for accrediting test laboratories. The Agent of the IGOSS Panel$^{*}$ shall establish technical criteria for laboratory accreditation. Technical experts for assessing laboratory competence (assessors) may be drawn from qualified Government, academic, industrial, or independent organizations.

The objectives of laboratory accreditation are to:

1) Identify technically competent testing services;

2) Assess and evaluate each test laboratory accredited to do testing for conformance to IGOSS by:

a) Conducting periodic laboratory proficiency testing to identify testing capability,

b) Initially, and periodically thereafter, conducting on-site assessments to determine compliance with the accreditation criteria, and

c) Conducting visits to verify reported changes in the laboratory's personnel, facilities, and operations, or to explore possible reasons for poor performance in testing practices; 
3) Insure that the test laboratory has adequate quality control, facilities, equipment and personnel to conduct testing;

4) Determine that the test laboratory staff is adequately trained in using the appropriate registered Means of Testing, following the prescribed conformance testing procedures;

5) Insure that adequate records are maintained to support the testing performed and that test reports are produced to provide the necessary information for determining conformance to IGOSS;

6) Notify the test laboratory of deficiencies;

7) Establish criteria and procedures for test laboratories to both obtain and maintain accreditation.

\section{Means of Testing Assessment}

Prerequisites of the methodology defined below are that an abstract test suite (ATS) exists, standardized or not, which has been publicly reviewed, with respect to the IGOSS PICS, amended as necessary, and is registered by the Agent of the IGOSS Panel*.

\subsection{Registered Abstract Test Suites}

A recognized abstract test suite shall be registered by the Agent of the IGOSS Panel* (hereafter called a registered ATS) upon approval from the OIW Conformance Testing SIG.

Amendment of an ATS is defined to be:

1) delete test cases which are not applicable to a profile;

2) specify constraints based upon agreements of the OIW which may be made either more rigorous or less rigorous;

3) specify additional test cases as necessary to encompass all mandatory and optional features using criteria stated in Part II of this Report;

4) Registration of the ATS shall be staged to accommodate improvements in the state of the art of test suite development. Each ATS will be harmonized with the ISO work and will reach stability with the International Standard. 


\subsection{Means of Testing Supplier}

Suppliers of a Means of Testing shall:

1) Request assessment of a product by submitting the forms and fees as required by an approved MOT assessment laboratory;

2) Identify the mapping between each abstract test case and the supplier's realization of it;

3) Arrange for a mutually satisfactory date for assessment of the MOT;

4) Demonstrate to the satisfaction of the MOT Assessment and Registration Authority, that the executable test cases achieve the test purposes and exhibit the dynamic behavior specified in the ATS when executed against an implementation of the corresponding OSI protocol;

5) Have a quality management program that assures maintenance and convergence of their product(s) such that the product(s) meet the requirements of registered ATS(s) and the Means of Testing Assessment and Registration Authority;

6) Have adequate mechanisms for distribution of updates and corrections to test laboratories employing the supplier products, in accordance with the Agent of the IGOSS Panel ${ }^{*}$ staged improvement procedures, and mechanisms to notify test laboratories of known problems in products.

\subsection{Approved MOT Assessment Laboratory}

An Approved MOT Assessment Laboratory shall:

1) Upon receipt of a request from an MOT supplier, and required fees, arrange for a mutually acceptable date for assessing the product;

2) Select one or more assessors from a group of experts who have no vested interest in the product and are neutral with respect to the results of the assessment, are technically competent with respect to the OSI protocol(s), IGOSS requirements, and the conformance test methodology employed;

3) Assess the Means of Testing using either a reference implementation, or an otherwise available implementation of the protocol(s) and following the procedures and criteria defined in Annex B and [JITC 1].

4) Upon completion of the Means of Testing Assessment provide the Means of Testing Test Report to the Registration Authority. 


\subsection{MOT Registration Authority}

The Agent of the IGOSS Panel* is the MOT registration authority. The Agent of the IGOSS Panel ${ }^{\star}$ has the authority to approve specific organizations that implements procedures and criteria equivalent to the ones defined in Annex $B$ to participate in the IGOSS MOT Assessment.

\subsection{Role of the MOT Supplier}

Using the test cases selected, the supplier shall:

1) provide the assessor with the facility to select and execute test cases on the MOT under assessment;

2) execute test cases selected by the assessor(s);

3) produce log files showing detailed protocol exchange behavior;

4) make these log files available to the assessor(s) for their analysis.

When the MOT has been registered, the MOT Supplier must provide a non-altered copy of the MOT Qualification Report to all its NVLAP and SCC Clients.

\section{REGISTERS EMPLOYED}

Essential to the operation of the provisions of this report are registers maintained by the Agent of the IGOSS Panel*. The names of the registers and a brief description of each are below.

\section{PICS Proformae for IGOSS}

For each IGOSS protocol a PICS-Proforma will be identified and registered . PICSProforma are used by OSI Product Vendors to make a statement of an OSI implementation, or system, stating which capabilities and options have been implemented, for a given OSI protocol.

\section{Abstract Test Suites for IGOSS}

For each IGOSS protocol a test suite composed of test purposes or abstract test cases is placed in the public domain and designated as the Registered Abstract Test Suite. The tests are updated from time to time to harmonize with International Standard test suites.

\section{Assessed Means of Testing for IGOSS}

For IGOSS profiles or substacks, the means of testing are assessed according to the 
criteria published in Annex B and in [JITC-1]. The treatment of derived implementations of a MOT is described in the documents. Any qualified system may be registered for use in IGOSS conformance testing.

\section{Approved Laboratories for Means of Testing Assessment}

An accredited laboratory that has been approved by the Agent of the IGOSS Panel* to conduct means of testing assessment. All the approved laboratories for means of testing assessment follow the procedures describes in Annex B.

\section{Laboratories Accredited for IGOSS Conformance Testing}

Any testing laboratory which complies with the provisions of this Special Publication and a companion handbook [NIST 16], as administered by the National Voluntary Laboratory Accreditation Program, may be accredited and added to the register. Compliance includes the operation of a registered Means of Testing realizing one or more registered Abstract Test Suites.

\section{Conformance Tested IGOSS Products}

IGOSS products which have been successfully tested by an accredited test laboratory using a registered Means of Testing, including registered Abstract Test Suites, may be added to the register. The treatment of derived implementations is described in clause 7.2.3 below and Annex C. Addition to this register is required before IGOSS interoperability testing is conducted.

\section{Interoperability Test Suites for OSI Products}

For each IGOSS application a test suite composed of test purposes is designated as the Registered Interoperability Test Suite. The tests are updated from time to time to achieve international harmonization.

\section{Reference Entities for Means of Testing Assessment}

For each IGOSS application or intermediate system and its supporting stack, one of which has been selected to support the Means of Testing assessment.

\section{Interoperability Test and Registration Services}

Any organization may offer to define procedures for the conduct of multi-vendor interoperability testing and to register the results of testing. Any such organization which is approved by the IGOSS Panel is entered onto this meta-register. The number of IGOSS approved Interoperability Services is not limited. General criteria for approval are given in Section 7.3.2.2. 


\section{EMPLOYMENT OF THE OSI CONFORMANCE TESTING METHODOLOGY}

ISO's OSI Conformance Testing Methodology and Framework [ISO 113] is an evolving multi-part international standard. It defines terminology, concepts, and requirements for: (1) other standards bodies who are responsible for producing abstract test suites; (2) suppliers of a means of testing (real test systems); (3) test laboratories; (4) clients of test laboratories; (5) information exchanged between test laboratories and their clients; and (6) proformae for test reports (whose content is outlined in Parts 4 and 5 of the ISO Conformance Methodology [ISO 113] and is detailed in standards associated with abstract test suites).

This standard provides the basis for the conformance aspects of the IGOSS testing program; in certain instances, however the work of ISO is not applicable to this report. For example, when:

1) Standardized abstract test suites do not exist for an IGOSS protocol,

2) Standardized abstract test suites do not test for features mandated by IGOSS,

3) ISO Conformance methodology does not address physical media, and

4) Interoperability testing is required.

The means of testing employed by accredited test laboratories may be a product of either the public sector or private sector, if the latter is a commercially available product.

This document references abstract test suites (or test methodology) for IGOSS which have been approved by the OSE Implementors' Workshop. They may be produced by the public sector, including standards bodies, or the private sector. Any registered abstract test suite or other test methodology employed shall be in the public domain, without protection of copyright.

This document provides criteria for assessment of the coverage and quality of test suites. As standardized abstract test suites, and their derived executable test suites, become available, they will be assessed for their adequacy under the criteria in this document, in order that they may be approved for use in testing IGOSS products.

Staged improvements to the abstract test suites will be conducted. The intention is ultimately to harmonize with International Standard OSI test suites.

\section{TESTING FRAMEWORK}

\subsection{Relation Between Testing Phases}

The immediate goal of testing communications products which claim to conform to the IGOSS specifications is to qualify them for inclusion in either the Register of 
Conformance Tested IGOSS Products, one of the Register of Interoperability and Registration Services, or both. The phases of testing coinciding with registration are conformance testing and interoperability testing, respectively.

1) Successful conformance testing by an accredited test laboratory using a registered Means of Testing, including registered Abstract Test Suites, leads to addition to the Register of Conformance Tested IGOSS Products.

2) Successful interoperability testing with other compatible products using a recognized methodology, that is, one on the Register of Interoperability Test and Registration Services, leads to publicly accessible documentation of pair-wise multi-vendor interoperability.

Requirements for IGOSS product suppliers to enter each phase of testing follow.

\section{Conformance}

1) Develop a IGOSS conformant product, or a partial stack thereof, which is testable using at least one of the methods specified here in.

2) Provide a PICS to an accredited test laboratory specifying functionality supported in the implementation for each protocol in the stack.

3) Provide a PIXIT to the accredited test laboratory for the stack/substack.

4) For stacks which build on substacks which have been previously conformance tested within the IGOSS testing process, provide an SCTR, PCTR and evidence of Registration for the previously tested functionality. For instance if the Session protocol is to be tested over transport class 4 protocol (TP4), an SCTR, PCTR and statement of registration should be furnished for the TP4 substack, as evidence that all the supporting protocols do not need to be completely resettled.

\section{Interoperability Testing}

Entry on the Agent of the IGOSS Panel ${ }^{*}$ register of conforming IGOSS products is a prerequisite to IGOSS interoperability testing.

Detailed mechanisms applicable to conformance and interoperability testing and the assessment thereof, are given in the following clauses. 


\subsection{Conformance Testing}

\subsubsection{What is To Be Tested}

Products of the following types may be made available for conformance testing:

- 7 layer Application stacks,

- 7 layer Relay stacks,

- 5-layer Session Stacks,

- 4 layer transport stacks,

- 2 or 3 layer Network stacks,

- 3 layer Intermediate System stacks.

This structuring explicitly recognizes test platforms (substacks) employed by existing testing technology.

In conformance testing, previously tested End System substacks may be carried forward into larger substacks. In such cases, comprehensive re-testing of previously tested functionality is not necessary: Basic Interconnection Testing is sufficient, providing that Protocol Conformance Test Reports are furnished for previously tested protocols. However, the same principle does not work in reverse. If a 7 layer stack is tested, using Single-layer embedded methods for the lower layers, and a substack is subsequently extracted and added as a component of a different 7 layer stack, then the whole of the new stack shall be comprehensively tested. This is because embedded testing alone does not provide sufficient confidence in a lower layer protocol when considered outside of its original stack context.

\section{Example:}

FTAM/ACSE/Presentation/Session/TP4/CLNP/LLC1/8802.3

Conformance testing of substacks may proceed as follows.

1) The subnetwork protocols (LLC1/8802.3) may be tested (although this is not separately mandated) and a System Conformance Test Report (SCTR) is produced and statement of registration may be produced.

2) TP4/CLNP is offered for testing over 802.3; the statement of registration is provided to demonstrate conformance to 8802.3. Basic interconnection testing is conducted to establish the basic workability of the substack. CLNP is tested by coordinated single-layer embedded means; TP4 is tested by coordinated single layer means. If conformance to both protocols is established, a second SCTR is produced for the substack. 
3) FTAM is offered for testing over TP4; the second statement of registration is provided to demonstrate conformance to TP4, CLNP and 8802.3. Basic interconnection testing is conducted to establish the basic workability of the stack (as an FTAM Initiator or as an FTAM Responder). Session, presentation and ACSE are tested by remote single-layer embedded means for use with FTAM Responders, and by distributed single-layer embedded means for use with FTAM Initiators. An FTAM Responder is tested by remote single-layer means; an FTAM Initiator is tested by distributed single-layer means. If conformance to all protocols is established, a statement of registration is produced for the FTAM Initiator and Responder stacks.

\subsubsection{How Testing Is Conducted}

These IGOSS Testing Guidelines are guided by the recommendations given in the OSI Conformance Testing Methodology and Framework [ISO 113]. MOT suppliers and conformance test laboratories are expected to be familiar with Part 5 which provides Requirements on Test Laboratories and Clients for the Conformance Assessment Process, and with the General Principles and Abstract Test Methods defined in Parts 1 and 2.

\subsubsection{Testing Elements}

For each stack supplied, the product configuration determines the test method used. Part 1 defines Abstract Test Methods which may be employed and provides guidance on their applicability to real test systems. Central to the concept of conformance testing is the ability to control and observe events of the Implementation Under Test (IUT) within the System Under Test (SUT). Every test method has, at minimum, a Point of Control and Observation (PCO) through the medium which connects the means of testing with the SUT. This is the point at which the means of testing injects valid and invalid Protocol Data Units (PDUs) of the protocol or protocols under test, and observes PDUs returned from the SUT. Any SUT which is accessible only through this PCO is testable using the Remote method. The distributed and coordinated methods provide extra control and coordination with the SUT, and this is usually effected through test coordination procedures which exist between the means of testing and the SUT. In all cases the initial stimulus for testing comes from the means of testing. For the remote method, the SUT is stimulated by protocol data units received via an underlying OSI service. For the distributed method, the SUT is stimulated directly at the $(N)$ service, by coordination interactions between the Means of Testing and the SUT. For the coordinated method the SUT is stimulated to generate (N)-PDUs as a result of prior interactions between the lower tester and upper tester, by means of a test management protocol.

The OSI Conformance Testing Methodology and Framework structures a test suite into Basic Interconnection, Capability and Behavior tests. The first two of these categories are proper subsets of the third (although the test purposes may be different). In the 
limit, Behavior tests provide exhaustive coverage - a limit which is by no means practical. Basic Interconnection tests are used in practical testing situations to check out the basic operation of the linkage between the SUT and the means of testing, and as such are not mandatory for coverage purposes. Capability tests are intended to provide 100 per cent 'breadth' of test coverage, i.e., at least one test per function specified in the protocol/Stable Implementor's Agreements for all mandatory and optional features. Behavior tests provide extra depth of coverage over all of the capabilities. In regard to coverage, options in a protocol are not optional in a test suite. Tests must be available for each option, even though they may not be selected for use with a particular IUT.

The administration of IGOSS testing includes recognition and registration of abstract test suites for each IGOSS protocol. Specific provisions are given in the Annex B.

\subsubsection{The Process}

The conformance assessment process includes:

- Preparation for testing,

- Test operations,

- Test report production.

The following clauses provide a brief description of these phases. A comprehensive description is given in Part 5 of IS 9646. Accreditation of conformance testing laboratories is directly based on the use of that test methodology.

\subsubsection{Preparation For Testing}

The preparation phase includes general documentation and configuration steps which must be carried out prior to conducting a test campaign. This includes furnishing of IGOSS PICS, PIXIT, and any vendor configuration requirements to a test laboratory.

\subsubsection{Test Operations}

The test operations phase includes static conformance assessment, test selection and parameterization, followed by dynamic testing. Test selection is based on options claimed to be supported in the IUT, as documented in the IGOSS PICS. Parameterization of selected tests is based on information provided in the PIXIT. Dynamic testing occurs using the executable realization of the selected abstract test cases, in which each test case is executed against the IUT to produce a Verdict. Any 'Inconclusive' verdicts may be resolved into 'Pass' or 'Fail' at this time.

\subsubsection{Test Report Production}

Test report production is a phase of assessment of the results of dynamic testing, and production of System Conformance Test Report and Protocol Conformance Test 
Report(s), recording the verdicts determined during the dynamic assessment.

\subsubsection{Addressing}

The testing of addressing includes a static check against the PIXIT for employment of the IGOSS addressing structure, and Basic Interconnection Tests to ensure that addressing is accurate.

\subsubsection{Evaluation of Conformance Testing}

Detailed criteria for the evaluation of conformance testing are provided by the Annex $B$ for test systems; [NIST、16], [NIST 26] and [SCC 1] for conformance testing laboratories.

\subsubsection{Treatment of Derived Products}

In certain circumstances a Product Registration may be derived from a tested 'base' implementation, without requiring further formal testing (see Annex A, A.1.2 and A.1.3). The following guidelines are applicable:

(1) In certain circumstances a IGOSS Product may be derived from a tested 'base' implementation, without requiring complete formal testing. In his application for derived product registration the vendor must provide the rationale for his assertion that the implementation is substantially unaltered (from the originally tested base product). When considering registration requests for derived products the Agent of the IGOSS Panel* will use the following guidelines.

(a) The host and target computer systems of the base and derived IGOSS Implementations have compatible instruction sets and operating systems. Common examples of compatible instruction sets and operating systems are two different computer system models in a manufacturer's product line or the computer systems produced by different manufacturers that use the same hardware mechanisms and operating systems.

(b) The IGOSS Implementation proposed for registration was derived from the base implementation by changes that are within the scope of accepted software maintenance practices. Arguments along this line should be included, in writing, with the application.

(c) Basic Interconnection Testing is conducted on the derived implementation, which results in the production of test logs showing PDU exchange activity which is the same as the base implementation or, if there are minor differences, these differences are justified as being within the scope of accepted software maintenance practices. Samples of the test logs and associated PCTRs for the basic interconnection tests should support the derived product application. 
(d) The base implementation was assessed in accordance with the Agent of the IGOSS Panel* procedures and is registered with the Agent of the IGOSS Panel*.

(2) An IGOSS Product may be registered for use over a different communications platform than that originally registered, without complete formal testing. When considering registration requests the Agent of the IGOSS Panel ${ }^{*}$ will use the following guidelines.

(a) The base implementation was assessed in accordance with the Agent of the IGOSS Panel* procedures and is registered with the Agent of the IGOSS Panel*.

(b) The communications platform to which the implementation is ported was assessed in accordance with the Agent of the IGOSS Panel ${ }^{\star}$ procedures and is registered with the Agent of the IGOSS Panel*.

(c) Basic Interconnection Testing is conducted on the ported platform which results in the production of test logs showing PDU exchange activity which is the same as the base implementation or, if there are minor differences, these differences are justified as being within the scope of accepted software maintenance practices. Samples of the test logs should support the ported platform application.

A derived or ported implementation may lose its registration if it is challenged successfully. Such challenges are described in Section A.2.

\subsection{Interoperability Testing}

The market and industry have matured significantly over the last couple of years to a point where interoperability testing, performed and managed according to formal methodology(ies) and in accordance with globally accepted practices, is seen more and more by major users and vendors as today's true complement to conformance testing. The objective here being "to provide a service which, for a specific protocol or set of protocols, will test whether two separate implementations are able to inter-work in a variety of operational conditions". The growing importance and understanding of interoperability is also increasing.

\subsubsection{What Is Tested}

Products made available for interoperability testing may be:

- 7 layer Application stacks,

- 7 layer Application Relay stacks,

- 3 layer Intermediate System stacks. 
In the case of End Systems, interoperability testing could proceed by pair-wise operation of compatible IGOSS systems. For instance, supplier A wishing to test an FTAM Initiator against supplier B should ensure first that B's product provides FTAM Responder capability with a compatible IGOSS profile. Moreover if B provides sender only, then A must be capable of receiving. It is for this reason that, in the same way as with conformance testing, a static analysis phase is necessary to determine whether testing can proceed at all.

Interoperability testing with Intermediate Systems (IS) operates on a multi-peer basis; pairs of End Systems communicate through one or more 3 layer Intermediate Systems. An IS must be capable of working with each End System on each supported subnetwork, and of routing data between pairs of End Systems. In a complex concatenated network, an IS must also route data from and to other ISs. Interoperability testing with Message Transfer Agent relay entities proceeds in a similar manner to Intermediate System testing, on a multi-peer basis.

\subsubsection{How Testing Is Conducted}

The interoperability testing requirements of U.S. IGOSS are grounded in the use of the following registers:

- $\quad$ the Register of Interoperability Test Suites

- the Register of Interoperability Testing Services

- the Register of IGOSS Compliant products

\subsubsection{Requirements of an Interoperability Testing Suite}

In order to be entered onto the register, an Interoperability Test Suite must:

1) be freely available (i.e., public domain or minimum costs of duplicating),

2) be subject to public review,

3) be capable of exercising the mandatory and optional functionality of each IGOSS application,

4) describe the purpose of each test and procedures by which the test purpose can be realized,

5) specify what constitutes a 'pass' or 'successful execution' for each test, and,

6) be supported by an organization recognized by the Agent of the IGOSS Panel*.

For each IGOSS application, only one Interoperability Test Suite may be registered. When a Test Suite becomes qualified it will be provisionally registered until the next IGOSS Version release. It will be reviewed and updated at that time. Staged 
improvements leading to harmonization proceed in this way until the completed Test Suite is fully registered. Registration remains current while the Implementation Agreements or the base standards remain valid. If more than one valid Interoperability Test Suite becomes available for a particular application stack, then one only will be selected by Agent of the IGOSS Panel* for registration.

\subsubsection{Requirements of an Interoperability Testing Service}

In the same way as with Conformance Testing, Interoperability Testing can be characterized as having the three phases of preparation, test operations and test report production. In order to become registered, any forum seeking to offer an Interoperability Testing Service should meet the following criteria:

1) Be a legal entity;

2) Make its Interoperability Testing Procedures available upon request to any user/supplier;

3) Use (at the minimum) a registered Interoperability Test Suite;

4) Conduct a static analysis phase which involves the selection of a common subset of the IGOSS tests including all of the mandatory tests and all the relevant optional tests resulting from the implementation of the same optional functionality;

5) Conduct a dynamic analysis phase in which both IGOSS product suppliers are in agreement concerning the outcome of each test. At the discretion of one or more of the IGOSS product suppliers, an Interoperability Testing campaign may be terminated before the Test Report is produced;

6) Issue a test report which identifies each IGOSS product supplier, describes the product including the supporting stack of protocols, and provides the list of all the possible test cases and identifies the tests selected and executed with a verdict for each test. The verdict may be 'pass' or 'fail'. Any fail verdict must be accompanied by an explanation outlining the cause of failure. Any non selected test case must be accompanied by an explanation outlining the rational for deselection.

7) Provide all the relevant information about the status of compliance for the product(s). If the product has passed conformance testing this information shall include the accredited laboratory where conformance testing took place, test campaign date and date of registration in the IGOSS Compliant Product Register.

8) At the request of the Agent of the IGOSS Panel*, a copy of the test report resulting from any bilateral or multilateral agreement made under the auspices of 
the Interoperability Testing Service. A nominal fee may be charged for each report supplied. Each IGOSS product supplier may require limitations on the use for which a test report is employed.

In cases where IGOSS users find a persistent lack of interoperability among products registered as interoperable, appeals may be made as follows:

1) To the vendor, or vendors of the inoperable products, who shall make every effort to make good on their warranty.

2) To the Interoperability Test and Registration Service who, after investigation may remove the product pair from the register.

3) To the Agent of the IGOSS Panel who may require to witness testing of either or both of the products involved, under the auspices of the Interoperability Test and Registration Service, in the original pairing, or in any other pairings required by the Agent of the IGOSS Panel ${ }^{*}$. In the event that the Agent of the IGOSS Panel $^{*}$ and the procuring Authority remain unsatisfied then the Interoperability Test and Registration Service may have its registration revoked.

\subsubsection{Treatment of Derived Implementations}

For the purpose of interoperability testing, no distinction shall be made for derived implementations. Any product registered should be subject to pairwise testing.

\section{RECOGNITION OF OTHER CONFORMANCE TESTING ACTIVITIES}

The IGOSS Partners seek to provide economical and adequate conformance testing. It is not the intent of the IGOSS Partners to duplicate conformance testing activities where those activities meet the Industry/Government requirements. Thus, the Agent of the IGOSS Panel ${ }^{*}$ will coordinate with other organizations to harmonize conformance testing requirements.

In meeting these objectives, the IGOSS Partners will consider the use of existing test methods, conformance testing procedures, test laboratories and certification systems.

Possible recognition of other activities include:

1) Foreign-based test laboratory accreditation and services,

2) Test method administration (maintenance and distribution systems),

3) Conformance testing procedures,

4) Test reports, 


\section{5) Certificates, and \\ 6) Test method research and development.}

The Agent of the IGOSS Panel ${ }^{*}$ may use any of the following methods for formally recognizing the conformance testing activities of other organizations:

1) Contract,

2) Accreditation,

3) Memorandum of Understanding, or

4) International Standard.

Any of the above methods are acceptable if they do not conflict with or compromise the Agent of the IGOSS Panel*'s authority in carrying out its responsibilities or violate Federal regulations.

Unless otherwise approved by the Agent of the IGOSS Panel ${ }^{*}$, agreements concerning conformance testing shall not:

1) Grant exclusive rights to others in fulfilling its responsibilities in the areas described above.

2) Unilaterally agree to adopt a product or service which conflicts with, or that does not allow for changes to meet, the IGOSS Panel requirements.

\section{THE QUALITY OF THE IGOSS TESTING PROGRAM}

The Agent of the IGOSS Panel ${ }^{*}$ is committed to upgrade/improve the IGOSS Testing Program to reflect inputs provided by the quarterly IGOSS/GOSIP Testing meetings and discussions with the European Community and several accreditation bodies in the world. The Agent of the IGOSS Panel ${ }^{\star}$ with the help of the IGOSS Partners intends to improve this program to be a leader in terms of quality, to provide useful information the public can trust, and to be attractive to vendors for both marketing and production quality improvements.

The IGOSS Testing program strives for continuous quality improvement in five key areas: Abstract Test Suites, Means of Testing, Laboratories, Product Registration, and Vendor Development Process. 


\subsection{Quality of Abstract Test Suites}

The Agent of the IGOSS Panel* has implemented a system of quality improvement to obtain completeness and suitability of the abstract test suites (ATSs) and to guarantee transparency in test campaigns.

The Agent of the IGOSS Panel ${ }^{*}$ will collect all the inputs (defect reports, unsuitable test cases) from accredited laboratories and from the OIW. The Agent of the IGOSS Panel ${ }^{*}$ forwards these reports to the relevant ATS maintenance authority as well as to all the NVLAP and SCC laboratories to insure transparency in the test campaign (i.e., same defects known by all laboratories).

\subsection{Quality of the Means of Testing}

The Agent of the IGOSS Panel ${ }^{*}$ has implemented a system of quality improvement to obtain completeness and suitability of the Means of Testing (MOTs) and to guarantee transparency in test campaigns(See Annex B).

The Agent of the IGOSS Panel* ensures that all MOTs are assessed by approved, accredited laboratories. Common procedures are in place to insure the same level of assessment and the same format for the "MOT Qualification Reports" based on the GOSIP Means of Testing Generic Test Plan [JITC 1]. NIST's Agent provides the MOT qualification reports to the MOT suppliers. The MOT suppliers are required to provide the relevant MOT Qualification Report to all their NVLAP and SCC laboratory clients. Moreover, the Agent of the IGOSS Panel ${ }^{*}$ collects any new defect reports from the IGOSS accredited test laboratories. The Agent of the IGOSS Panel* consolidates these defect reports and provides them as feedback to the MOT suppliers. MOT suppliers are required to provide these new defect reports to their NVLAP and SCC laboratory clients. This system helps to achieve transparency in test campaigns and to facilitate harmonization of test reports.

\subsection{Quality in the Laboratories.}

All NVLAP and SCC accredited laboratories are required to maintain their procedures fully compliant with ISO Guide 25 [ISO-114] and the relevant element of ISO 9002 [ISO-9000]. NVLAP and SCC monitor the quality and competence of the IGOSS Testing Laboratories. 


\subsection{Quality of the Product Registration}

The Agent of the IGOSS Panel is the Product Registration Authority. The Agent of the IGOSS Panel I $^{*}$ as in place a comprehensive and high-quality test report review process. The objectives of this review are twofold: 1) the suitability of the products to the relevant profiles and 2) the guarantee that the laboratories perform to the level of quality required (see Annex $A$ ).

During this process, the registration authority verifies that all the documents provided by the vendors follow the procedures defined in ISO 9646 [113] and comply to the IGOSS Testing program requirements. Indeed, a test report must be comprehensive, complete, reliable. Some of the documents required include the Protocol Implementation Conformance Statement (PICS), Protocol Implementation eXtra Information (PIXIT), Protocol Conformance Test Report (PCTR), System Conformance Test Report (SCTR), Product Architecture Diagram, and Test Configuration Diagram. A block and wire diagram must be provided that includes: 1) a pictorial explanation of the product architecture which accurately depicts the product to be registered and 2) an explanation of the test configuration which accurately describes the software, hardware, and protocols used by each components of the product. Each test case executed must result in a behavior acceptable from the point of view of the relevant International Standards and Stable Implementation Agreements. When a test case does not behave as expected, the laboratory must demonstrate that no instance of non-compliance occurs and provide all necessary technical justifications and logging information.

\subsection{Quality in the Vendor Development Process}

Vendors that have been assessed for quality (i.e., ISO 9000) may be able to enter their status in the IGOSS Register Database. The plan would allow four status levels: Unknown, ISO 9001, ISO 9002, and ISO 9003.

ISO 9000 (Quality Management and Quality Assurance Standards [ISO 108]) focuses on the quality system in place in the vendor organization in various elements of the product life-cycle (i.e., design, development, production, final inspection and test, installation, and servicing). The Introduction Section of ISO 9000 states:

"A principal factor in the performance of an organization is the quality of its products or services. There is a world-wide trend towards more stringent customer expectations with regard to quality. Accompanying this trend has been a growing realization that continual improvements in quality are often necessary to achieve and sustain good economic performance. 
Most organizations - industrial, commercial or governmental - produce a product or service intended to satisfy a user's need and requirements. Such requirements are often incorporated in 'specifications'. However, technical specifications may not in themselves guarantee that a customer's requirements will be consistently met, if there happen to be any deficiencies in the specifications or in the organizational system to design and produce the product or service. Consequently, this has led to the development of quality systems standards and guidelines that complement relevant product or service requirements given in technical specifications. The series of International Standards (ISO 9000 to ISO 9004 inclusive) embodies a rationalization of the many and various national approaches in this sphere".

At the time of registration, together with the PCTRs and SCTRs, vendors would be asked about the quality level of the vendor organization. The Agent of the IGOSS Panel ${ }^{*}$ would require the proof of any claimed quality registration. The relevant ISO 9000 certification body would also be referenced. It is not the role of the IGOSS Testing Program to assess the quality and validity of these bodies.

This recognition of the quality in the Vendor Development Process is not yet implemented. 


\section{ANNEX A: IGOSS PRODUCT REGISTRATION}

Note: In case of conflict, the most updated and approved version of the product registration procedure take precedence on the information provided in this Annex. Contact the Registration Authority for the latest procedure.

\section{A.1 Registration Information}

\section{A.1.1 Summary: How to Become Registered}

A product cannot legitimately be claimed as IGOSS Compliant if it is not registered. The conditions for registration are given below.

1) To be registered a product must have been successfully conformance tested in a NVLAP accredited laboratory (or a laboratory which is affiliated through a Memorandum of Understanding) or an SCC accredited laboratory. The testing requirement applies to all IGOSS protocols within the product, with exceptions as noted below.

2) A partial stack product may be registered if:

a) all IGOSS protocols within it are successfully conformance tested (e.g. P2, P1, RTS, Session); and

b) the connectivity platform(s) referenced is also registered (e.g. TPO/X.25 PLP/X.25 HDLC LAP B).

3) The testing and registration of LAN products $(8802 / 2,8802 / 3,8802 / 4,8802 / 5)$ is optional; however, untested two-layer LAN products may not be legitimately claimed as IGOSS compliant, whereas successfully tested protocol stacks running above untested two-layer LAN products can be legitimately claimed as IGOSS compliant. This provides the exception to rule 1), illustrated as follows: In a full stack platform of P2/P1/RTS/Session/TP4/CLNP/8802.2/8802.3, CLNP through P2 must be tested and registered. The LAN connectivity 8802.2/8802.3 must be referenced in the product application (as a specific product identification) but need not exist as a product on the register of tested LANs.

4) There is no testing, or registration requirement for physical layer protocol products.

5) Derived products may be qualified for registration according to the criteria given in section A.1.3 of this document.

Product suppliers with IGOSS products to be tested and registered should:

1) Complete the IGOSS PICS, or set of PICSs, for the protocols implemented in the product. 
2) Submit the product for conformance testing to an IGOSS registered laboratory.

3) Ensure that all executed tests are PASSed. Tests Not Run/Selected are deselected according to the relevant criteria.

4) Submit the PICSs, PCTRs and SCTR together with the IGOSS Product Registration application form (obtainable from Agent of the IGOSS Panel ${ }^{*}$ or from the conformance laboratory) to the Agent of the IGOSS Panel* for review and registration.

\section{A.1.2 Base Products}

The definition of a IGOSS "product" has been engineered to allow the register to retain a coherent structure. Of course a supplier is at liberty to package products in his own way, but this means that it is possible that what a supplier packages as one product may need to appear as entries on multiple registers. The principle concern of the IGOSS Testing Program is that protocol functionality at each layer be conformance tested, and it is this aspect which determines whether a product is a "Base".

To clarify: a Base Product is that part of a supplier's packaged software product which implements IGOSS functionality and has successfully passed conformance testing, and resides on a computer system with a named operating system, or a family of computer systems having the same processor and instruction set and the same operating system as used during the test campaign.

\section{A.1.3 Derived Product Guidelines}

(1) In certain circumstances a IGOSS Product may be derived from a tested 'base' implementation, without requiring complete formal testing. In his application for derived product registration the vendor must provide the rationale for his assertion that the implementation is substantially unaltered (from the originally tested base product). When considering registration requests for derived products the Agent of the IGOSS Panel ${ }^{*}$ will use the following guidelines.

(a) The host and target computer systems of the base and derived IGOSS Implementations have compatible instruction sets and operating systems. Common examples of compatible instruction sets and operating systems are two different computer system models in a manufacturer's product line or the computer systems produced by different manufacturers that use the same hardware mechanisms and operating systems.

(b) The IGOSS Implementation proposed for registration was derived from the base implementation by changes that are within the scope of accepted software maintenance practices. Arguments along this line should be included, in writing, with the application. 
(c) Basic Interconnection Testing is conducted on the derived implementation, which results in the production of test logs showing PDU exchange activity which is the same as the base implementation or, if there are minor differences, these differences are justified as being within the scope of accepted software maintenance practices. Samples of the test logs and associated PCTRs for the basic interconnection tests should support the derived product application.

(d) The base implementation was assessed in accordance with the Agent of the IGOSS Panel* procedures and is registered with the Agent of the IGOSS Panel ${ }^{*}$.

(2) An IGOSS Product may be registered for use over a different communications platform than that originally registered, without complete formal testing. When considering registration requests the Agent of the IGOSS Panel ${ }^{*}$ will use the following guidelines.

(a) The base implementation was assessed in accordance with the Agent of the IGOSS Panel* procedures and is registered with the Agent of the IGOSS Panel ${ }^{*}$.

(b) The communications platform to which the implementation is ported was assessed in accordance with the Agent of the IGOSS Panel ${ }^{*}$ procedures and is registered with the Agent of the IGOSS Panel*.

(c) Basic Interconnection Testing is conducted on the ported platform which results in the production of test logs showing PDU exchange activity which is the same as the base implementation or, if there are minor differences, these differences are justified as being within the scope of accepted software maintenance practices. Samples of the test logs should support the ported platform application.

A derived or ported implementation may lose its registration if it is challenged successfully. Such challenges are described in Section A.2.

\section{A.1.4 Derived Product Guideline Analysis}

One of the most significant activities in the IGOSS program is that of placing products on the IGOSS register. The IGOSS program provides two types of registration; base and derived. The "base" registration is the initial registration for a product and is achieved only after the product has been fully tested by an accredited lab. The "derived" registration can be obtained when a vendor can definitively prove that a subsequent version of that product is essentially unchanged from its base version. This is done without the product having to undergo the same levels of testing as for a base registration. 
The purpose of this section is to provide both general and, where possible, specific guidelines for derived registrations. In the attempt to quantify the conditions for derived registrations it was determined that the problem is too complex to allow for any reasonably simple guidelines. The greater success has been in guidelines for specific situations which comprise only a subset of the possibilities. As vendors identify new situations each will be examined individually and ruled upon by the registration authority. Input, suggestions, and disagreements relative to this topic is requested.

It is the position of the Agent of the IGOSS Panel* that in order to register an implementation as a derived product it must be demonstrated that there are no significant differences between it and the base product from which it is being derived. The process of determining this deals with three significant entities called the protocol engine, the virtual machine, and the underlying stack (i.e., connectivity).

The protocol engine is that portion of the product that is defined by the PICS and tested by an accredited test lab for IGOSS conformance. It is the heart of the registered product and cannot be modified in any way without requiring a complete re-test for a subsequent IGOSS product registration. Therefore, the initial condition for a derived registration is that the PICS for the new product version is identical to the PICS for the base registered version. Any change in the PICS implies a change to the protocol engine which requires complete conformance testing.

The virtual machine is the environment in which the protocol engine operates and consists of the hardware platform and the operating system. Changes to the virtual machine which affect the protocol engine (i.e., not transparent) will not be allowed without a complete re-test of the candidate product. All changes to the virtual machine will have to be carefully reviewed before a derived registration can be allowed. It is incumbent upon the product vendor to provide complete justification of any claims for derived registration when the virtual machine is affected.

The underlying stack, or connectivity, is that set of software, firmware, and hardware that provide the services required by the protocol engine to connect through the LAN or WAN to its peer(s). For example, TP4 can connect through CLNP over 802.2 (LLC) over 802.3 (MAC). The two changes that occur relative to the substack are connectivity or implementation (i.e., same protocol, but different product) changes. All changes to the underlying stack (connectivity) will also have to carefully reviewed before a derived registration can be allowed.

In order for the Registration Authority to grant a derived registration for a product that meets the requirement that the change(s) be transparent to the protocol engine, the vendor must provide both conclusive proof of that the change was transparent and a demonstration of testing at the "derived" level. This "derived" test suite will be a subset of the ATS which is sufficient to provide confidence that the claim for a derived registration is valid. The results of this test must be provided to the Registration Authority in the same PCTR format used for base registrations. 
Where an ATS or MOT does not exist (e.g., 802.4, 802.5) with which to provide this "derived" test it will be necessary for the vendor to perform an "interoperability" test between the base and derived product. This test should be conducted using the appropriate "mandatory" OSINET tests for two high level protocols, such as MHS or FTAM. A description of the test and results must be provided in the place of the "derived" test results.

Unless otherwise specified in a policy statement, no product will be considered for a derived registration without the test report from a successful "derived" or interoperability test, irrefutable written evidence that the protocol engine has not been affected by whatever modification is being considered, and a PICS that is the same as the one for the base product. The following are examples of circumstances where derived registrations may or may not be considered given that all of the previously defined conditions have been met.

Registrations Relative to the Virtual Machine: Changing the virtual machine, which is made up of the hardware and operating system, requires complete conformance testing. Usually, changing the operating system (e.g., DOS to UNIX), system calls, or the platform affect the protocol engine and are generally excluded from derived registrations. However, some cases have been identified in which hardware or operating system changes might occur without changing the virtual machine as viewed by the protocol engine.

Derived registrations will be considered for platform changes if there is conclusive evidence presented by the applicant that the protocol engine is unchanged because it is isolated by the operating system from the platform architecture (e.g., the POSIX operating system), but care should be given to consider timing changes.

Example:

product/POSIX/systemX $==>$ product/POSIX/system $Y$

Derived registrations will be considered, without testing, if there is conclusive evidence presented by the applicant that there is no difference in the hardware architecture. For example, the difference in model numbers is due to different peripherals being configured with the same processor, chassis, etc.

Example:

product/OpSys/platformX.1 ==> product/OpSys/platformX.2

Derived registrations will be considered if there is conclusive evidence presented by the applicant that there were minor modifications in the operating system (e.g., minor version changes) which were transparent to the protocol engine.

Example:

product/OpSysX.1/platform ==> product/OpSysX.2/platform 
Registrations Relative to Interface Changes: Derived registrations will be considered where the substack is changed from one registered substack product to another when the protocol connectivity remains unchanged. In other words, given that two, or more, identical registered products are available for a given protocol it is allowed to change from one to another and to derive the registration of the second stack since the connectivity remains unchanged.

\section{Example:}

$$
\text { TP4/CLNP }(\text { brand } x) / L A N==>\text { TP4/CLNP }(\text { brand } y) / L A N
$$

CLNP Registration Relative to MAC Sub layer Changes: Because the CLNP protocol is insulated from MAC changes it is possible to "derive" register a conformant CLNP product from the MAC $(802.3,802.4,802.5)$ it was tested over to another MAC given that the LLC sub layer remains unchanged.

\section{Example:}

CLNP/802.2/802.3 ==> CLNP/802.2/802.4

CLNP Registration Relative to LAN/WAN Changes: Because the SNDCF (Sub Network Dependent Convergence Function) is tested with the CLNP it should be considered part of the CLNP which excludes derived registrations from LAN to WAN or visa versa. The purpose of the SNDCF is to create a generic interface between CLNP and the Service Provider (LLC1 or X.25). Because the SNDCF differs between LANMAN, the connectivity change is not transparent to the CLNP protocol engine.

An investigation of CLNP shows that when it is implemented over LLC that the LLC assumes the bulk of the SNDCF functionality. In this situation the SNDCF is a simple interface which passes the CLNP requests and LLC responses back and forth between the CLNP and the LLC with whatever format changes are required. When CLNP is implemented over X.25 the SNDCF provides a significant amount of functionality by both translating the requests and providing the $X .25$ connection control. It is obvious that there is no similarity between the X.25 SNDCF and the LAN SNDCF and hence CLNP/LLC and CLNP/X.25. Since no mechanism exists to isolate the SNDCF for testing it is necessary to require a Base registration for this connectivity change.

TP4 Registration Relative to CLNPMNAN CLNP/LAN Changes: Because the TP4 protocol is insulated from the WAN/LAN changes it is possible to derive register a conformant TP4 product from the LAN or WAN it was tested over to the other given that the CLNP sub layer was tested and registered over both.

There are two conditions under which this might happen. One is that the CLNP was only registered over one of the substacks when the TP4 was tested and registered over it. The other is that the CLNP was registered over both substacks, but the TP4 was only tested and registered over one. In the first case, the TP4 could be derived to the additional substack after the CLNP was "re-registered" over both substacks. In the 
second case, the TP4 could be tested and registered over one substack and derived to the other.

Example A:

(1) base registration TP4/CLNP/LAN (CLNP only over LAN)

(2) CLNP is tested and registered for both LAN and WAN

(3) $==>$ TP4/CLNP/WAN

Example B:

(1) base registration TP4/CLNP/LAN (CLNP over LAN \& WAN)

(2) $==>$ TP4/CLNP/WAN

TP4 Registration Relative to CONS (X.25) and CLNS (CLNP): Because there is a difference in protocol behaviors between TP4/CONS (TP4/X.25) and TP4/CLNS (TP4/CLNP) it is not allowed to do a derived registration between them. TP4/CONS and TP4/CLNS are generally separate implementations of the protocol engine.

Registrations Relative to TP4 to TP2/TP0 Changes: Because there is a difference in protocol behaviors between TP0, TP2, and TP4 it is not allowed to do a derived registration between TP4, TP2, or TP0.

FTAM Registrations Relative to TP4,TP2 and TP0: Because the Service Provider interface provided by the transport layer appears to be the same for TP0, TP2 and TP4 it is possible to derive register an FTAM/TPO product from either an FTAM/TP2 or FTAM/TP4 product or derive an FTAM/TP2 product from an FTAM/TP4 given that the Transport product are both IGOSS registered.

Example:

\section{FTAM $/$ TP4/CLNP ==> FTAM/TPO/X.25}

Product Registrations Relative to User Interface Changes: Because the protocol engine is insulated from most User Interface changes it is possible to derive register a product from a conformant base given that the protocol engine is unchanged.

Product Registrations Relative to Version Changes: Because product version changes are often due to modifications transparent to the protocol engine it is possible to derive register a product from a conformant base given that the protocol engine is unchanged.

\section{A.1.5 Mutual Recognition Arrangements}

An IGOSS product registered or certified by another authority may be registered with the Agent of the IGOSS Panel ${ }^{\star}$ if:

(a) A Memorandum of Understanding exists between the Agent of the IGOSS Panel $^{*}$ and the registering or certification authority, recognizing each other's assessment and registration procedures. Among other things this MOU is 
dependent on mutual recognition arrangements between NVLAP and corresponding accreditation bodies.

(b) Specific application is made to the Agent of the IGOSS Panel* by the IGOSS product supplier. (Automatic concatenation of registers will not occur).

(c) The candidate IGOSS product for registration passes the Agent of the IGOSS Panel ${ }^{*}$ static evaluation.

(d) The candidate IGOSS product has not been removed from the Agent of the IGOSS Panel* register as a result of a successful challenge which has not been resolved by the primary registration or certification authority.

If the above conditions are met, the candidate IGOSS product will be registered. If a IGOSS product which is registered under the provisions of a Mutual Recognition Arrangement is removed from the primary authority's register then it shall be removed from the IGOSS register.

The Agent of the IGOSS Panel ${ }^{*}$ will not require full testing of implementations assessed by a mutually recognized authority. The Agent of the IGOSS Panel ${ }^{*}$ will review the application for indications of satisfaction of IGOSS requirements. The IGOSS Panel is the final authority on whether, and in what circumstances, registration shall occur.

Applications which are acceptable to the Agent of the IGOSS Panel ${ }^{*}$ are entered in the register of IGOSS conformance tested products under the same categorization as the primary registration (i.e., Registered or Provisionally Registered) and with the same constraints - Registration is based on the given Registered Abstract Test Suite, The IGOSS product supplier will be notified when registration is approved. The register will be maintained up-to-date by the Agent of the IGOSS Panel* and the information will be available according to the procedures given in Annex $\mathrm{C}$.

\section{A.1.6 Product Register Structure}

Most OSI products are multi-layered so each separately identified product, as defined by the supplier, is registered according to the highest layered protocol which it contains. For every protocol for which IGOSS compliance is claimed, a PCTR must be provided. This must either be submitted as part of the product for which registration is sought, or referenced as part of a registered platform. For Local Area Network interfaces underlying CLNP, a non-IGOSS supporting platform will be considered on a case-bycase basis. These circumstances will only occur in certain platforms supporting CLNP.

A product may be composed of one or more Testable Units. Testable Units are defined as: Product $=$ "exposed" Testable Unit

+ (zero or more) "embedded" Testable Unit(s)

+ Supporting/ Registered Platform 
Register categories are numbered P-1 through P-n. The following list is provided for information only and reflects the numbering for GOSIP Version 2.

P-1 WAN Products:

X.25 PLP + HDLC LAP B

CONS/X.25 PLP + HDLC LAP B

ISDN

P-2 LAN Products:

8802.2 LLC + 8802.3 MAC

8802.2 LLC + 8802.4 MAC

8802.2 LLC + 8802.5 MAC

P-3 Intermediate Systems:

CLNP + Supporting LAN/WAN Platforms

CLNP+ES-IS + Supporting LAN/WAN Platforms

P-4 Transport Products:

TP4 + CLNP + Supporting LAN Platform(s)

TP4 + CLTP + CLNP + Supporting LAN Platform(s)

TP4 + CLNP + Registered WAN Platform(s)

TP4 + Registered WAN Platform

TPO + Registered WAN Platform

P-5 Session Products:

Exposed Session + Registered Transport Platform(s)

P-6 X.400 Products:

P1 + RTS/Session + Registered Transport Platform

P1 + RTS + Registered Session Platform

$\mathrm{P} 2$ + P1 + RTS/Session + Registered Transport Platform

$\mathrm{P} 2$ + P1 + RTS + Registered Session Platform

$\mathrm{P} 2+$ Registered P1 Platform

P-7 FTAM Products:

FTAM/ACSE/Presentation/Session + Reg. Transport Platform

FTAM/ACSE/Presentation + Registered Session Platform

P-8 Virtual Terminal Products:

VTPIACSE/Presentation/Session + Reg. Transport Platform

VTPM/ACSE/Presentation + Registered Session Platform

Register fields are:

Supplier Name: 


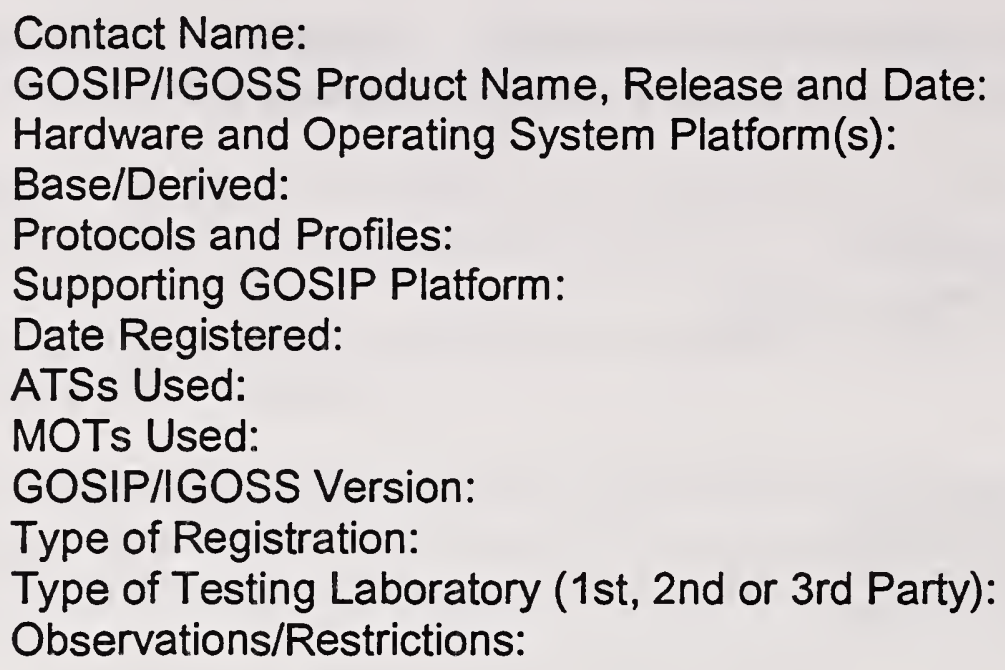

\section{A.1.7 Registration Categories}

Products must be successfully conformance tested in a NVLAP or SCC Accredited laboratory (or a laboratory which is affiliated through a Memorandum of Understanding), using registered Means of Testing. The number of products registered is not limited. Categories are:

Registered - Valid for the life of the product or until major revision. Based on fully registered Means of Testing and fully registered Abstract Test Suites.

Provisionally Registered - Valid for the life of the product or until major revision, or upgrade to Full Registration by testing against a Fully Registered MOT. Based on provisionally registered Means of Testing and/or provisionally registered Abstract Test Suites.

\section{Discussion}

The difference between Fully Registered and Provisionally Registered products is in the quality of testing undergone. Initially, products are tested against Provisional MOTs and thus are Provisionally Registered themselves. When the MOTs are upgraded to Full Registration, Products subsequently tested implicitly provide a higher assurance of IGOSS compliance. It is at the vendor's discretion whether and when a product shall be upgraded by re-testing. Provisional and Fully Registered products coexist on the register, but new procurements are advised to favor Fully Registered products, wherever possible.

The Agent of the IGOSS Panel* intends to make registers of IGOSS conformant products available to the public through quarterly publication and on-line access. If the supplier objects to such procedures, a written statement should be included with each application.

\section{A.1.8 IGOSS Implementation Philosophy}


In establishing the registration scheme for the IGOSS testing program, the Agent of the IGOSS Panel* was compelled to structure the conformance tested products register according to the 'testable units' of the 7-layer OSI stack. This structure may seem to constrain the implementation choices of a IGOSS product supplier, but that is not the intention of the IGOSS Panel, and not at all the spirit of OSI. The nature of distributed implementation implies the possibility that what OSI defines as an "End System" may be implemented over multiple processors. The nature of the interprocess communication between layers within an OSI stack, and between an OSI stack and its user interface, may be tightly or loosely coupled, and is not constrained by the IGOSS specification. Examples of acceptable OSI implementation strategies are:

1) A multi-processor system implements transport on a "front-end" and applications (FTAM->Session, for instance) on separate systems, communicating over a bus or a LAN.

2) A network file server implements FTAM and supporting OSI protocols on one system, with user access by multiple workstations using any local means of interprocess communication including communicating over a LAN.

3) In a messaging system, the Message Transfer Agent is implemented on one system and User Agents are deployed in separate workstations. The MTAs and UAs communicate over a LAN.

\section{A.2 Appeals}

If an Acquisition Authority finds a defect in a registered IGOSS product, then the primary recourse must be to the product supplier. If the Acquisition Authority remains dissatisfied, then details may be given to the Agent of the IGOSS Panel ${ }^{*}$, and the product supplier may be required to demonstrate conformance with respect to a given test or tests. If the supplier is unable to demonstrate conformance in those cases, then the product's registration may be withdrawn. For each test or set of tests appealed, the appellant is required to pay a nominal fee.

In the case of a derived product, if the supplier is unable to demonstrate conformance with respect to any tests, then the derived registration may be revoked. In such a case, a derived registration cannot be reinstated: the product must undergo a complete conformance test, to achieve registration as a base implementation.

\section{A.3 Related Conformance Testing Information}

Prior to entry onto the register of IGOSS Conformance Tested Products it is necessary for a product to be successfully conformance tested in a NVLAP or SCC accredited laboratory, using an IGOSS registered Means of Testing. IGOSS Testing Program policy has been to promote improvements in the underlying testing infrastructure; in 
practice this involves migration of Abstract Test Suites, and updating of Means of Testing, with the result that earlier ATS and MOTs become obsolete. It is important for a product supplier to know the ATS and MOT obsolescence policy before attempting registration - indeed, before initiating formal testing.

Related to the testing improvement issue is the treatment of defects in Abstract and Executable test suites. Registration can occur only after the appropriate tests have been selected and successfully executed. It is important for both the test laboratory and the Registration Authority to have the same picture of Abstract and Executable defects. ISO 9646 assumes perfection in all test cases - and the IGOSS Testing Program is painfully aware that the situation is otherwise. Hence, additional procedures for the de-selection of test cases are given below.

\section{A.3.1 Test Case De-selection Procedures}

Test de-selection may be based on: 1) non-implementation of optional capabilities, 2) inapplicability of tests for IGOSS. Tests selected but not run may be based on: 3) Abstract Test Case defects, 4) Ambiguities and Errors in the Standards leading to Abstract Test Case defects, 5) Executable Test Case defects (generally applicable, and acknowledged by the MOT supplier), and possibly 6) Executable Test Case defects (specific to an IUT, and maybe acknowledged by the MOT supplier).

A review of the application of the test case de-selection mechanisms is appropriate:

1) Non Implementation of Optional Capabilities

- De-select.

- Optional capabilities not claimed need not be tested, of course. Note however that some options may be IGOSS Requirements. Non implementation of these optional capabilities will result in failure of Static Conformance.

- Optional capabilities implemented must be tested, if a test case exists, except for de-selection based on points 2) to 6).

2) Inapplicability of Test for IGOSS

- De-select.

- This situation exists in cases where the test suite was developed in Europe based on a European profile, and IGOSS points to a version of the OIW Implementors Agreements which is not aligned. A review of the Abstract Test Cases in 1990 was supposed to highlight these kinds of problems, but that review was not sufficiently effective. 
Any such tests identified today may be de-selected, with written justification of non-applicability. The registration authority will generate a list of all such tests. These tests are excluded from what constitutes "100\%" in determining whether an MOT supports the required threshold percentage of coverage.

\section{3) Abstract Test Case Defects}

\section{(a) Serious Abstract Test Case Defects}

- $\quad$ Selected and Not Run.

- Any such tests identified today need not be run, based on written justification of an ATC defect from the registration authority. The registration authority maintains a list of all such tests.

- $\quad$ These are tests which are "temporarily excluded" from the requirement for execution, pending the application and promulgation of a "fix" by the appropriate Test Suite maintenance authority.

\section{(b) Trivial Abstract Test Case Defects}

\section{- $\quad$ Selected and Run}

- $\quad$ Any Abstract Test Cases which are labeled as defective, but for which the defect is superficial, or for which a valid workaround exists, must be run. If necessary, verdicts may be assigned after manual analysis. The registration authority maintains a list of all such test cases.

4) Ambiguities and Errors in the Standards leading to Abstract Test Case Defects

- $\quad$ Selected and Not Run.

- Any such tests identified today need not be run, based on written justification of non-applicability. This justification should include an interoperability impact statement, since it is likely that such specification problems will be variously treated by different product implementors. It may be useful to distinguish between "positive" testing and "negative" testing here, due to their possibly different interoperability consequences:

The purpose of positive testing is to determine that an IUT can initiate good protocol, and respond correctly to good protocol. The problems of ambiguous specification, when not tested, or incorrectly tested, can result in serious interoperability consequences. Testing laboratories and IGOSS test report reviewers are advised to carefully consider these consequences prior to registration. 
The purpose of negative testing is to determine that an IUT can respond correctly/in a well-mannered fashion to errors initiated by the MOT. This usually results in either ignoring the offending PDU, or initiating an abort sequence. In general, the problems of ambiguous specification, when exercised by negative testing, are more likely to be self-resolving by resulting in an abort.

The registration authority is advised to generate a list of all such ambiguous specification areas and associated tests for submission to the relevant standards making authorities, including the OSE Implementors' Workshop.

- $\quad$ These are tests which are "temporarily excluded" from the requirement for execution, pending the application and promulgation of a "fix" by the appropriate Test Suite maintenance authority. These tests are included in what constitutes "100\%" in determining whether an MOT supports the required threshold percentage of coverage.

\section{5) Executable Test Case Defects}

(a) Serious Executable Test Case defects (generally applicable, and acknowledged by the MOT Supplier and the Registration Authority)

- $\quad$ Selected and Not Run.

- $\quad$ Any such tests need not be run, subject to written justification and corroboration by the MOT Supplier. This is the purpose of having the MOT Supplier issue synchronized bug lists to their users and to the Registration Authority.

- $\quad$ It is the responsibility of the MOT Supplier to fix these tests.

(b) Trivial Executable Test Case defects (both general and specific)

- $\quad$ Selected and Run

- $\quad$ Any Executable Test Cases which are labeled as defective, but for which the defect is superficial, or for which a valid workaround exists, must be run. If necessary, verdicts may be assigned after manual analysis. The registration authority coordinates lists of all such test cases, for every MOT.

- It is the responsibility of the MOT Supplier to fix these tests. 
6) Serious Executable Test Case defects (specific to an IUT, and acknowledged by the MOT Supplier and the Registration Authority)

- $\quad$ Selected and Not Run.

- $\quad$ This is possibly the thorniest category of all, and most subject to potential abuse. Many of the errors in this category may be alternatively dealt with under the "Re-Assignment of Verdict" rubric. Any such errors which are not acknowledged by the MOT Supplier may be reviewed on a case by case basis, and accepted or refused by the Registration Authority, based on the best available technical opinion. A different weight may be placed on the claims depending on whether it is a "positive" or a "negative" test which is sought to be withdrawn. Any such errors may also be brought before the OIW CT and relevant protocol SIGs, whose decision will be considered by the IGOSS Testing Program.

will be accepted by the IGOSS Testing Program. It may be the responsibility of the MOT Supplier to fix these tests.

\section{A.3.2 The Distinction Between "Serious" and "Trivial" Errors}

Any Abstract or Executable Test Case defect detected by the Conformance Laboratory must be either listed with the MOT Supplier and the Registration Authority a priori, or else a Discrepancy Report (DR) submitted to the Registration Authority upon the discovery of the error (and its seriousness), prior to the submission of the test report for registration. The Registration Authority will rule on the validity of the DR according to the best technical opinion available, including if necessary, a review under the terms of the OIW CT SIG dispute resolution process.

Any test listed as defective or discovered to be defective must be attempted to run and a manual verdict assessed. If the test purpose is not achievable, then the error is "Serious" and the test may be marked as "Selected and Not Run". If the test purpose is achievable, then the relevant verdict may be recorded in the PCTR.

\section{A.3.3 Reassignment of Verdicts}

IS 9646 indicates that Pass or Fail verdicts in an abstract test case may not be varied by the test laboratory. This is obviously an ideal situation where mature, standardized test suites are in use, which is not the case today, further, it does not take into account imperfect mappings between abstract and executable test cases. The situation today is that many of the abstract test suites have serious defects which require critical review with respect to the protocol standards and Implementors Agreements. In these cases, ongoing review by the test laboratories is necessary to improve the test suite standards. Many of the executable test cases have defects, some of which are discovered by the MOT Validation Laboratory. 
In the case of executable defects, the verdict should be changed to comply with the associated abstract test case.

In the case of abstract test case defects, the verdict should be determined in accordance with the Stable Implementors' Agreements and/or the International Standard. The registration authority will periodically produce addenda to the ATS to clarify problems discovered therein.

\section{A.4 Product Registration Checklist}

\section{Reliable Test Report(s)}

The Test Report, as a record of the products behavior, must be reliable to the point that a potential Customer can determine the product's capabilities. In order to do this the report must be readable, describe what was tested versus what capabilities were claimed, and precisely identify the substack over which it was tested.

\section{Reproducibility of Tests}

The test reports must provide adequate information about the test configuration and conduct to allow for the reconstruction and execution of the test with predictable results. In order to do this the test configuration must be specified in the PCTR, SCTR, PICS, and PIXIT. This includes a detailed specification of the hardware, software, and substack connectivity (i.e., protocol(s), software, communication boards). (The SCTR, PCTR, and PICS may reference only the hardware/software configuration that the product was tested on.)

When a single "base" application is for multiple hardware platforms, operating systems, etc. it is essential that each virtual machine be equivalent to the tested system. These additional platforms should only be referenced in the application with information appended to the application that absolutely proves that the equivalencies. They should not be referenced in any way in the SCTR or the PCTR.

The SCTR, PCTR, PICS, and PIXIT are the official record of the product test. The Registration Authority application form is for administrative purposes and registration clarification. The application is not used or referenced relative to the product assessment, nor provided to acquisition authorities. In other words, the SCTR, PCTR, PICS, and PIXIT, not the application, represent the testing of the products' functionality and, by themselves, should provide sufficient detail that the test can be reconstructed and consistently repeated, at any time, with the same results. This makes it mandatory that these documents be accurate, complete, and provide the details of the test configuration (hardware, software, etc.) and test results. 
The application, which is not part of the permanent record, does not supplant nor supersede the SCTR, PCTR, PICS, or PIXIT. The information provided by the application may be used to clarify the form which the Register entry may take, but will not modify any information or claims found in the SCTR, PCTR, or PICS. The product assessment is based solely upon the information in the SCTR, PCTR, and PICS. It is the policy of the Registration Authority not to assume or to guess the details of the testing or product configuration. If the required information is not in the respective documents then the application stands to be rejected.

The PICS and PIXIT provide the declaration of the product's abilities and a specification of certain software configurations which need to map to the actual test case selections. If a capability is claimed in the PICS then it shall be tested unless a disclaimer appears in either the PICS or PCTR stipulating that the capability was not to be tested and the reason why (e.g., future capability, current capability not being claimed, CLNP over LAN instead of X.25, ATS/ETS not available).

Manual verdict assignments must be justified in the observation section of the PCTR. All observations must be both interpretable and meaningful plus provide adequate evidence to substantiate whatever claim is being made. Copies of the test log are required.

(3) Test Conduct Adheres to the Relevant Standards

The form and format of the testing must be per the Agent of the IGOSS Panel* documented procedures, the appropriate Stable Implementation Agreements, ISO 9646, the appropriate Protocol Standard(s), and accepted test practices.

(4) Test Determined that the Product Meets the Standards (ISO, SIA)

The test must completely test the product's functionality, regardless of the state of the ATS and/or MOT(s). All broken or defective ATCs/ETCs with workarounds shall be executed and testing shall be to the International Standard. Deficiencies in the ATS or MOT are not a loophole to be used to avoid testing product functionality. This extent of testing is the responsibility of the product vendor and is in keeping with the philosophy that the Registration Authority represents the Buyer of the US IGOSS products.

The test results shall be correct with all manual verdicts fully documented. All verdict changes must be consistent with relevant standards and be justified by the Registration Authority. Every ATC must be accounted for, by ATC name, as either Deselected; Selected, but Not Run; or Run with a verdict of Pass, Fail, or Inconclusive. The inconclusive verdict requires sufficient justification for its existence, as well. 
(5) Test Laboratory Demonstrated Adequate Quality Assurance Procedures

All test laboratories are NVLAP or SCC accredited which requires that they have a living Quality Manual and Quality Assurance practices. The test laboratory shall:

(a) Provide Document Tracking such that the SCTR, PCTR, PICS, and PIXIT have unique identifications assigned to them, which are consistent throughout the documentation.

(b) Provide A PCTR based on the ATC names rather than the ETC names. The ATS is relatively constant while the ETCs vary from MOT to MOT.

(c) Provide evidence of a QA program by the neatness and readability of the test report documents.

(d) Provide Test Case Counts that match the total of ATCs in the ATS and the verdicts. The Test Case Count should be equal to the number of test cases De-selected plus test cases selected, but not run plus test cases with pass, fail, and inconclusive verdicts.

(e) Provide Documents and Applications that avoid the assumption of the reader's familiarity with the product(s). Product Information Specifications should contain the following information in a clear and understandable format such as:

Vendor Name:

Product Name:

Version/Release:

Operating System:

Hardware Platform:

(f) Ensure that relevant entries in the documents match those that are in the IGOSS Register (e.g., MOT names, Test Laboratory names and addresses, ATS identification). Include the NVLAP or SCC number when referencing the test laboratory.

(g) Ensure completeness of the documentation such that all the information required to assure product description and test reproducibility is provided. The following, information relative to the test environment, should be contained in the SCTR and/or the PCTR.

Protocol(s) Tested:

Test System Name:

Test System Version Number:

Connectivity: (e.g., 802.2/802.3 or 1984 X.25 PLP/HDLC Lap B, RS-232) 
Dates of Testing:

MOT Release Date:

(h) Ensure consistency of information throughout the documents such that there are no contradictions or discrepancies.

(i) Ensure that the Underlying Stack is on the current US IGOSS Register and that it is completely specified in the SCTR and/or PCTR. This includes the following information in a clear and understandable format such as:

Connectivity: (e.g., 802.2/802.3 or 1984 X.25 PLP/HDLC Lap B, etc.) Product Name: (i.e. name or identification of the software product or package that implements the underlying stack)

Op Sys: (i.e. operating system that the named Underlying Stack product executes under)

Hardware: (i.e., specific name and/or detailed description of the communication board or hardware needed for connectivity)

NOTE: When the Underlying Stack is a LAN it is not necessary that it be registered in order to claim IGOSS conformance, but it is still mandatory that it be accurately and completely identified with this information.

(j) Ensure that $100 \%$ of mandatory test cases were selected.

(k) Ensure that $100 \%$ of the optional test cases were selected for implemented optional functions.

(I) Ensure that all test cases that were selected, but not run are justified by the registration authority.

(m) Ensure that all new ATC/ETC problems are submitted to the Registration Authority for review so that a decision can be made prior to the application for registration.

(n) Ensure that any test cases that are de-selected are justified as for either optional functions which are not implemented or for non-applicable test cases.

(o) Ensure that everything claimed in the PICS is tested or that a disclaimer exists that specifies that the capability is not being claimed and why.

(p) Ensure that the final verdicts are clearly displayed in the PCTR.

(q) Ensure that all of the verdicts presented in the SCTR and PCTR fall within the allowed classifications: (1) Pass; (2) Selected, but not run; (3) 
Inconclusive; (4) De-selected; or (5) Failed. No other verdicts are recognized by the IGOSS Testing Program.

(r) Ensure that there are no failure verdicts recorded. The IUT must pass every applicable test.

(s) Ensure that there are no blank verdicts for those test cases that were selected and run.

(t) Ensure that no test cases were run that do not appear in the registered ATS.

(6) Minimization of Assessment Costs and Duration

In order to maintain or reduce the cost of doing product assessments and registrations as well as reducing the time required to perform them, the following should be done:

(a) Provide high-quality test reports.

(b) Provide complete explanations per observations or justifications. If the Registering Authority finds it necessary to do the research then the assessment time and cost will increase.

(c) Provide all of the required information. The more information and details provided by the vendor, the less time the Registration Authority has to devote to research or contacting the applicant for information.

(d) DO NOT attempt to contact and/or badger the Registration Authority personnel regarding the assessment's progress. Frequent inquiries into the status of a registration only results in the prolonging of the assessment. The Registration Authority will do product assessment on a first come (i.e., payment received), first serve basis and will call the vendor regarding any questions or issues. Plan well ahead on submissions so as to provide sufficient time before deadlines to handle any submission discrepancies that might occur. Failure to plan on the part of the vendor does not constitute a crisis on the part of the registration authority. 


\section{ANNEX B: MEANS OF TESTING VALIDATION}

Note: The technical information concerned with the Means of Testing validation methodology presented within this Annex is provided for information only. The actual reference for the IGOSS Means of Testing Validation Methodology is the latest approved version of [JITC-1].

\section{B.0 . Tutorial}

The IGOSS Means of Testing Assessment and Registration requirements are being changed to reflect needed improvements in test system quality and to formalize some practices which have developed in the day to day management of the register. The motivation for the changes is to enable more rigorous product testing standards, within the limit of the state of the art.

When NIST/CSL first established the GOSIP Testing Program in 1990, the state of MOT technology was quite variable, with some systems achieving high quality and a high percentage of test suite coverage, and other systems with many outstanding software problems and a relatively lower level of test suite coverage. The Abstract Test Suites upon which coverage was assessed varied in quality and coverage. At that time we deliberately chose to accept MOTs into the program having a minimum coverage of $60 \%$ of the available ATS.

The situation today, in 1993, is that there is now a well established MOT registration program, for all types of GOSIP Version 2 protocols. Many problems have been found in the initially registered Abstract Test Suites, and we are ready to accept improved ATS for many protocols from the Open Systems Testing Consortium (OSTC) who remain the principal support for test suites which are tailorable to U.S. GOSIP requirements. It is now appropriate to establish the MOT assessment program for IGOSS Version 1 MOTs. Test suites for some of the Version 1 protocols, such as X.25 Packet and HDLC LAP B, are at the IS/DIS levels in ISO. MOTs based on these tests may be fully registered, assuming at least $95 \%$ coverage.

Other changes to the assessment and registration program include the following:

1 Any MOT operating over an X.25 platform must be able to interoperate with IUTS which implement the 1984 standard capabilities. This is in support of the requirement that an IUT operating over X.25 must be able to demonstrate at least Basic Interconnection in order to be registered for that platform. It is accepted that this does not necessarily imply that the MOT itself must support X.25 (1984). It would be inappropriate to make that requirement since many Wide Area Networks do not support 1984 capabilities. 
2 The ability for MOT suppliers to provide minor enhancements or bug fixes which do not change the test case coverage without requiring a full assessment.

3 The ability for MOT suppliers to register incremental improvements in test case coverage and bug fixes without a complete Dynamic Validation by the MOT Validation Laboratory. The MOT supplier must provide documentary evidence of regression testing and copies of test case logs for additional or improved test cases. This also opens the door for recognition of the results of an equivalent (or better) MOT Validation organization - subject to the establishment of a Memorandum of Understanding.

4 A complete dynamic assessment which requires exercise and scrutiny of $100 \%$ of those test cases which are relevant to GOSIP, in the registered Abstract Test Suite.

5 Points 2-4 are also predicated on the requirement to show proof that the changes are reflected in improved product documentation.

These changes are not without cost - particularly the move to $100 \%$ validation. It is reasonable for the cost of a full MOT validation to be more than the cost of a commercial conformance testing campaign, since the effort and the skills involved are considerably greater. This cost is mitigated by the three tiered structure of the assessments and registrations involving (1) nominal change, (2) static assessment and (3) full dynamic assessments for which the fees will also be tiered accordingly. Actual responsibility for determining the fees belongs to the Approved MOT Validation Laboratories.

\section{B.1. Introduction}

The program of MOT assessments and registration is administered by the Agent of the IGOSS Panel ${ }^{\star}$ who has authority delegated by the executive director of the IGOSS Panel. The accepted means of testing validation methodology for the IGOSS is the most updated version of [JITC 1].

\section{B.1.1 Purpose}

This Annex explains the technical and administrative procedures for the assessment of MOTs for GOSIP protocols. The assessments are conducted by technical experts. MOTs which are found to be valid are registered by the Agent of the IGOSS Panel ${ }^{*}$ and for use in Conformance Test Laboratories which are seeking NVLAP or SCC Accreditation.

In certain circumstances, MOTs showing some deficiencies from the requirements may be provisionally registered - and recommended for Conformance Test Laboratory use pending rectification of the deficiencies. 


\section{B.1.2 Method}

ISO IS 9646 OSI Conformance Testing Methodology and Framework describes methods and a process by which implementations or partial implementations of 7-layer and 3-layer OSI stacks may be tested for conformance to the base protocols. The realization of test methods as Means of Testing generates a further testing problem: by what method shall a Means of Testing itself be validated? A framework for the execution and interpretation of results is required. The problem is compounded by the fact that no Reference Implementation can exist without comprehensive and systematic testing, which itself cannot occur without a Means of Testing.

The solution adopted for the GOSIP Testing program is a bootstrap method. The initial candidate Reference Implementation is executed against the initial candidate MOT, using the process defined in 9646-5, and the results interpreted in two ways:

1) conventionally, to assess the candidate Implementation Under Test (IUT) response, the results are checked against the Abstract Test Suites.

2) the MOT is statically checked against the criteria given in the main part of this document; and the results of execution are used to assess the MOT's encoding and decoding integrity, and its faithful mapping of the Executable Test Suite with respect to the Abstract Test Suite.

After the candidate Reference Implementation has been conformance tested, the known bugs are catalogued. If it meets the operational criteria, the Reference is provisionally registered (as a "Reference Implementation" and NOT as an IGOSS conformance tested product, since in fact the Reference may not meet the criteria for entry onto the tested products register), and may be employed in the MOT assessment and registration program, using the method described in this document.

The prerequisites for the application of this method of MOT assessment are highquality Abstract Test Suites, and a sufficient expertise in OSI protocol and test technology.

\section{B.1.3 Field of Application}

This Annex is addressed to:

(1) Suppliers of IGOSS Means of Testing wishing to achieve Agent of the IGOSS Panel $^{\star}$ registration.

(2) Suppliers of OSI test services seeking accreditation as an IGOSS Conformance Test Laboratory, and requiring to use the Agent of the IGOSS Panel ${ }^{\star}$ registered Means of Testing. 
(3) Technical experts, who use the procedures herein to conduct MOT assessment campaigns.

(4) Other MOT assessment authorities which seek to enter into mutual recognition and common registration arrangements.

\section{B.2. Summary of the Assessment process}

\section{B.2.1 The Summary}

(1) The Agent of the IGOSS Panel* announces the availability of Standard Reference Materials (SRMs) for the testing of particular IGOSS protocols. SRMs include Abstract Test Suites (Internationally standardized or not).

(2) The MOT supplier applies to a registered Validation Laboratory for information. The Validation Laboratory supplies application forms, reference entity configuration information (if available), PIXIT proforma and information about the process of validation.

(3) The MOT supplier completes the application form and provides to the Validation Laboratory: ATS/ETS mapping, ETS defect list, baseline or regression test report, user documentation, tester PIXIT information, test log files (in the case of a version update), and the appropriate fees.

(4) The MOT Validation Laboratory reviews the application and may perform a full static review of the MOT supplier's claims.

(5) If the MOT passes the static review it may be directly registered, in the case of update to a previously registered MOT. Otherwise a complete dynamic validation is necessary. A date and a site for the dynamic validation will be negotiated.

(6) The MOT Validation Laboratory assessor conducts a dynamic review and makes a report to the MOT supplier, the IGOSS Registrar, and the Agent of the IGOSS Panel*.

(7) Based on the results of the validation the MOT may be registered, or registration may be deferred pending corrections. The complete list of registration possibilities is given in section B.5.2 below.

(8) Candidate IGOSS Conformance Test Laboratories which are seeking NVLAP or SCC Accreditation select MOTs from the IGOSS Register. Conformance Test Laboratory accreditation and registration will be based on Registered or Provisionally Registered MOTs only. 


\section{B.2.2 Routes to Registration}

\section{B.2.2.1 Tiers}

A three-tiered assessment scheme has been established for MOTs because the review/assessment effort is different according to what is being registered, and what level of preparation the MOT supplier has applied prior to application. The three tiers therefore reflect costs for the Validation Laboratory and the Registration function, and are:

$\begin{array}{ll}\text { - } & \text { COMPLETE Assessment } \\ \text { - } & \text { PARTIAL assessment } \\ \text { - NOMINAL check }\end{array}$

The Complete Assessment may be required under some of the following conditions:

- $\quad$ IGOSS version change;

- $\quad$ Abstract Test Suite coverage (\%) requirement increase;

- $\quad$ Abstract Test Suite change;

- $\quad$ Provisional to Full registration change;

- Insufficient confidence in product or testing;

- Major changes in connectivity.

Four processes are involved in the complete assessment:

A) Manual review of test reports against a baseline IUT supplied by the vendor.

B) Full Dynamic Assessment of the MOT involving

1) A manual review of:

a) Claimed coverage of ATS,

b) Mapping of Executable Test Cases versus ATS,

c) Mapping of PICS to test case selection.

2) Execution of all ETC's.

3) Evaluation of the test logs for all ETC's Versus ATS.

4) Assessment of coverage of ATS.

C) Manual review of the product documentation provided by the vendor.

D) Manual review of defect reports supplied by the vendor. 
The Partial Assessment involves the manual review of test reports supplied by the vendor and the submissions of the test logs from new test cases or modified test cases of the MOT against the baseline IUT. An updated defects report is also required. A partial assessment would be required for:
- $\quad$ A revision for a MOT version update;
- Platform change;
- Mutual Recognition Arrangement;
- Transparent changes in connectivity.

The Nominal check involves a documentary review, including evidence that the change does not require partial or complete testing. In principle, a nominal check is for a cosmetic change that does not significantly affect the test execution engine. The vendor must send a notification and/or application plus required fees.

\section{B.2.2.2 Objects}

The general apprach is that objects which can be entered onto the MOT register, and their required levels of assessment, are as follows:

1) Base Product (Complete)

A previously unregistered MOT, or any MOT which warrants a full dynamic assessment.

2) Derived Connectivity (Complete)

Derived from a currently registered Base MOT, based on change of supporting connectivity that requires protocol changes, such as LAN instead of WAN for CLNP.

3) Unverified Functional Update (Complete)

An MOT change which involves alteration to testing functionality or improved test coverage which is NOT adequately corroborated by supporting test log files and documentation.

4) Derived Product (Partial)

Derived from a currently registered (Base) MOT, based on some change of hardware or operating system.

5) Derived Substack (Partial) 
Derived from a currently registered (Base) MOT based on change of supporting connectivity that does not alter the protocol's behavior, i.e. TPO instead of TP4 under FTAM.

6) Verified Functional Update (Partial)

An MOT change which involves alteration to testing functionality or improved test coverage, which is corroborated by supporting test log files and documentation. i.e. MOT Version update.

7) Mutual Recognition Arrangement (Partial)

An MOT registered with another authority recognized by the Agent of the IGOSS Panel*, for which IGOSS registration is sought.

8) Cosmetic Update (Nominal)

A version change which involves no alteration to testing functionality or the test coverage, and a change that does not affect the protocol engine, i.e. user interface changes.

\section{B.3. Static Assessment}

\section{B.3.1 Goals of Static Assessment}

1) To determine whether an MOT claims support of IGOSS functionality and provides at least the minimum required test coverage.

2) To determine whether the user documentation adequately describes installation of the MOT, Selection, parameterization, execution, results analysis and verdict assignment for each test case; also, that the documentation describes the correspondence between executable test cases and the registered Abstracts, and that all test case defects are fully described.

3) To determine whether a Partial or Complete Dynamic Assessment is necessary.

\section{B.3.2 Process}

(1) Check that all forms and fees are supplied and completed.

(2) Check the Means of Testing capabilities against the generic requirements in Appendix III of this document. 
(3) Determine the coverage of the Executable Test Suite using the method described in B.3.3.2 below. Determine which tests are claimed as defective, and their percentage with respect to the total number of tests.

If the MOT has been recognized under a Mutual Recognition Arrangement, determine whether its coverage is valid for IGOSS.

(4) Determine whether it is appropriate to proceed with Partial or Complete dynamic assessment.

\section{B.3.3 Test Suite Issues}

\section{B.3.3.1 Abstract Test Suite Validity}

Abstract Test Suites are selected from a variety of different sources, and sometimes at arbitrary levels of coverage and quality. These circumstances combine to determine the 'state of the art' for the precision of conformance testing. It is desirable to have this level increase in precision, and at the same time to have the ATS formalized to become International Standards.

The mechanism has been to select and review ATS based on the current version of IGOSS, and update the ATS in conjunction with successive version releases of IGOSS. The limiting factor on this improvement process has been the amount of effort available to fix bugs in the Abstract Test Cases and to add more test cases to improve the coverage. This process of test suite management will probably not stop even after the IS level has been reached for any particular test suite - since the standardization of a test suite has no bearing on its validation through experience of use.

\section{B.3.3.2 Coverage Guidelines}

In order to assess the Test Suite coverage for the MOT there are two possibilities:

(I) There exists a Registered Abstract Test Suite for the MOT,

(II) There does not exist a Registered Abstract Test Suite for the MOT.

In the case where a Registered Abstract Test Suite exists, method (I) must be chosen; otherwise, method (II) must be chosen.

(I) Compare, or cross reference the Executable Test Suite against the Abstract Test Suite to establish coverage, in terms of breadth and depth.

Breadth: there shall be one Executable Test Case corresponding to each IGOSS function in the PICS. All IGOSS functionality must be testable.

Depth: there shall be one Executable Test Case for each function tested by the Abstract Test Suite. Note, this does not require a one to one correspondence 
between ETCs and ATCs: one Executable Test Case may be implemented to realize more than one test purpose.

(II) Using a IGOSS PICS as a basis, apply the coverage criteria given in ISO IS 9646-2, section 10.4, and reproduced here:

(a) for Capability test groups:

(1) at least one test purpose per relevant capability;

(2) at least one test purpose per relevant PDU type and each major variation of each such type, using normal or 'default' values for each parameter;

(b) for test groups concerned with test event variation in each state: at least one test purpose per relevant state/event combination;

(c) for test groups concerned with timers and timing, at least one test purpose concerned with the expiry of each defined timer;

(d) for test groups concerned with encoding variations, at least one test purpose for each relevant kind of encoding variation per relevant PDU type;

(e) for test groups concerned with valid individual parameter values:

(1) for each relevant integer parameter, test purposes concerned with the boundary values and one randomly selected mid-range value;

(2) for each relevant bit-wise parameter, test at least one value which is not the 'default' value;

(3) for other relevant parameters, at least one test purpose concerned with a value different from what is considered 'normal' or default in other test groups;

(f) for test groups concerned with invalid individual parameter values:

(1) for each relevant integer parameter, test purposes concerned with invalid values adjacent to the allowed boundary values plus one other randomly selected invalid value;

(2) for each relevant bit-wise parameter, test purposes for as many invalid values as practical; 
(3) for all other relevant types of parameter, at least one test purpose per parameter;

(g) for test groups concerned with combinations of parameter values:

(1) at least one test purpose for each important combination of specific values (e.g., boundary values);

(2) at least one test purpose per set of interrelated parameters to test an 'unusual' combination of relevant values."

\section{B.4. Dynamic Validation}

This section provides the procedures for conducting a dynamic review of a IGOSS MOT. Since the intention of this assessment procedure is to determine compliance of MOTs with IGOSS testing requirements, there will be no extraneous diagnostic assessment or recommendations. Assessors are directed not to inquire too deeply into the underlying reasons behind non-compliance; it is sufficient to note the occurrence. An MOT supplier may opt to negotiate exit from the assessment process at any time.

\section{B.4.1 Goals of Dynamic Validation}

1) To develop confidence that an MOT provides test coverage at or above the current threshold level, and to document defects in coverage so as to allow accurate review of test reports prior to product registration.

2) To corroborate a claim of improved test coverage.

\section{B.4.2 Prerequisites}

\section{B.4.2.1 Validation Laboratory Responsibilities}

1) that a Reference, or known implementation, be installed and operational on a separate system. This shall be under the control of the MOT Validation Laboratory. It is preferable that the Validation Laboratory has tested Reference Implementations, but there is the special case of a first time MOT, or the case where a suitable Reference is not available to the Validation Laboratory.

2) that the Registered Abstract Test Suite be available for validation of results. This will be used in conjunction with the Abstract Test Case defects list.

\section{B.4.2.2 MOT Supplier Responsibilities}

1) that the MOT, including all associated tools, be installed on a system and be operational; 
2) that the MOT supplier provides a conformance log and SCTR/PCTR for an IUT of their choice, to be used as a baseline for the assessment.

\section{B.4.2.3 Shared Responsibilities}

1) that the static analysis of the Reference and of the MOT has been performed prior to the assessment. 


\section{B.4.3 Dynamic Assessment Procedures}

\section{B.4.3.1 Complete Assessment}

(1) Establish Basic Interconnection between MOT and System Under Test.

(2) Conduct a review of the MOT documentation, to determine whether an MOT can be configured and operated without direct assistance from the MOT supplier.

(3) Validate the characteristics of the MOT from generic characteristics given below. For each specified characteristic, select and execute a test and assess the MOTs support of that characteristic. For instance, in CLNP, the characteristic "capability to construct CLNPDUs according to both the fully segmenting and the non-segmenting subsets, and send them to a CLNP end system under test over any supported medium". Select tests which involve the generation of fully segmented and non-segmented CLNPDUs. Using log file analysis tools and IS 8473 , determine that the format and content of one sample of each CLNPDU is correct.

Another example: in FTAM, the "capability to negotiate and encode specified document types". Select and execute a test which includes the use of the document type FTAM-3. Using log file analysis tools and IS 8571-2, determine that the format and content of each FADU is correct.

(4) Using the Static Assessment results from Section B.3, above, validate the Executable Test Suite for the MOT. There are two possibilities for this procedure:

(I) There exists a Registered Abstract Test Suite for the MOT: demonstration of the equivalence of: the results of execution of an Executable Test Case, and one path through each associated Abstract Test Case (if more than one). Note, all tests are executed, independently of the supplier's claims, since this is primarily a test of the MOTs capabilities and not those of the System Under Test.

(II) There does not exist a Registered Abstract Test Suite for the MOT: validate the results of execution of each Executable Test Case, by comparison against the base standard and relevant Implementor's agreements. Note, all tests are executed, independently of the supplier's claims, since this is primarily a test of the MOTs capabilities and not those of the System Under Test.

\section{Generic MOT Characteristics}


1) The capability to analyze PICS and PIXIT for the IUT and to select and parameterize tests to be run, and to configure the means of testing for communication with the SUT.

2) Procedures to reconcile PDU and test data with the test purposes and yield a verdict for each test purpose.

3) The capability to produce Conformance Test Reports, listing test cases executed and their verdicts, and detailing the IUT behavior in cases of failure.

4) Capability to record the protocol data units exchanged with the IUT in a conformance log, and to review the structure and encoding of protocol data units after the test campaign is complete.

5) The capability to construct PDUs for the appropriate protocol and send them to an attached IUT.

6) The capability to receive and decode PDUs according to the appropriate protocol specification. The capability to validate PDUs received and record the results in a conformance log.

7) The capability to construct invalid as well as valid PDUs.

8) The capability to monitor and initiate exchanges of PDUs between the Means of Testing and the IUT (inopportune as well as normal).

\section{B.4.3.2 Partial Assessment}

(1) Establish Basic Interconnection between MOT and System Under Test.

(2) Using the Static Assessment results from section B.3, above, for every (formerly defective) test case claimed as fixed, validate the test case using one of the following two methods:

(I) If there exists a Registered Abstract Test Suite for the MOT: demonstrate the equivalence of the results of execution of an Executable Test Case, and one path through each associated Abstract Test Case (if more than one).

(II) If there does not exist a Registered Abstract Test Suite for the MOT: validate the results of execution of each Executable Test Case, by comparison against the base standard and relevant Implementor's agreements.

\section{B.4.4 Assessor's Report}


After completion of the static and dynamic aspects of the Assessment, the assessor completes the MOT Qualification Report, one copy of which goes to the MOT supplier and one copy to MOT Registration Authority. The MOT supplier is required to provide a non-altered copy of the MOT Registration Report to all its NVLAP and SCC clients.

The completed assessor's report consists of:

- The results of Static Assessment,

- The results of Dynamic Assessment,

- The corroborated list of test case defects,

- The calculation of percentage coverage,

- The assessor's recommendation for registration.

The MOT supplier may choose to disagree with any portion of the report by appending his signed comments indicating the contested portion(s). This appeal may be made directly to NIST, who will inquire into the reasons for the disagreement and may corroborate or overturn the Validation Laboratory's decision. Technical issues may be referred to the OIW for their consideration.

\section{B.5. Registration}

\section{B.5.1 Fee Basis}

Fees are charged for MOT Validation and Registration based on the work required to determine an MOT's validity. This is based on the "Objects" of section B.2.2.2.

\section{B.5.2 Categories and Criteria for Registration}

The following actions may be taken by the MOT Registrar:

(1) Registration of the product

(2) Provisional Registration of the product

(3) Deferral of Registration pending corrections

(4) Denial of Registration

(5) Revocation of Registration

(6) Expiration of Registration

(7) Withdrawal by the MOT Supplier.

The criteria for assignment to each category are:

(1) Registration of the product

(a) operation of the MOT over a Registered IGOSS platform, i.e., that part of the MOT stack which provides OSI connectivity shall have been successfully tested and registered as a IGOSS conformant product 
(Unless valid arguments to the contrary can be made that this need not be the case).

(b) correct implementation of $95 \%$ or more of the Registered Abstract Test Suite(s). Note, the ATS must be fully registered, not provisionally.

Both conditions (a) and (b) must occur before registration can be granted. Registration is granted until the expiration of registration of the associated Abstract Test Suite (or the earliest such expiration in the case of multi-layer testers tested using embedded methods), or until withdrawal of the product by the MOT supplier.

\section{Provisional registration of the product}

(c) operation of the MOT over a working IGOSS platform, i.e., that part of the MOT stack which provides OSI connectivity is able to interoperate with the System Under Test in the context of a test campaign.

(d) correct implementation of not less than the current threshold percentage of the Registered Abstract Test Suite, if one exists. If a Registered ATS does not exist then the determination of what constitutes "100\%" is determined by the Agent of the IGOSS Panel ${ }^{*}$, using the ISO guidelines given in B.3.3.(II), above and the percentage rule is applied to this figure. This percentage may be varied from year to year to stage progressive improvement in the installed testing and IGOSS implementation bases.

(e) Provisional Registration will not occur if one or more MOTs for the same protocol or profile are already fully registered.

If conditions (c) and (d) occur then Provisional Registration may be granted. Provisional Registration is granted until 6 months after the next ATS is registered. The MOT supplier may apply for registration based on an improved percentage coverage at any time during the currency of the associated ATS.

\section{(3) Deferral of Registration}

If agreement has been reached between the MOT Validation Laboratory and the MOT supplier, then after the assessment the supplier may make minor changes to the MOT which, with acceptable documentary evidence of the fixes, may be accepted for deferred registration.

\section{(4)}

\section{Denial of Registration}

Registration may be denied for any of a variety of reasons, including the following: 
- failure of the MOT to interoperate because of a defective IGOSS platform, i.e., that part of the MOT stack which shall provide connectivity is not able to interoperate with the System Under Test.

- correct implementation of less than the current threshold percentage of the Registered Abstract Test Suite.

- $\quad$ the MOT is unable to execute a test campaign without crashing, or requires frequent initialization.

- $\quad$ product documentation is inadequate.

- the list of known defects is not provided.

\section{(5) Revocation of Registration}

If an MOTs test case coverage falls below the threshold percentage, due to the discovery of executable test case defects, the MOT supplier is given 90 days to provide enough fixes to ensure that the threshold percentage is exceeded. If within 90 days rectification has not occurred, as demonstrated by a Static Registration, the MOT is removed from the register. After this time no products tested against the affected MOTNersion will be entered onto the Conformance Tested Products Register.

The first subsequent version of the same MOT shall undergo a complete dynamic validation before entry onto the register.

\section{(6) Expiration of Registration}

When an MOT is fully registered there is normally no expiration date associated with it. This is however contingent upon the stability and quality of the associated fully registered ATS.

In the provisional situation, Abstract Test Suites are periodically updated and registered, and MOTs are revalidated and registered in synchronization with these, usually with a higher threshold percentage coverage requirement. In cases where the registered ATS and the percentage coverage requirement does change, existing registered MOTs shall expire 6 months after the registration of MOTs based on the upgraded coverage requirement. No product tested against an expired MOT version will be entered onto the Conformance Tested Products Register.

\section{(7) Withdrawal by the MOT Supplier}

An MOT version which is registered may be withdrawn at any time by the MOT Supplier. After this time no products tested against it will be entered onto the Conformance Tested Products register. In addition, products originally tested using this MOT which are entered on the Conformance Tested Products Register may be 
annotated with an indication that the MOT against which they were tested has been withdrawn.

\section{B.5.3 Derived Implementation Guidelines}

The following conditions are suggested as guidelines for determining whether a IGOSS Means of Testing may be derived from a base implementation:

(a) the host and target computer systems of the base and derived IGOSS MOTs have compatible instruction sets and operating systems. Common examples of compatible instruction sets and operating systems are two different computer system models in a manufacturer's product line or the computer systems produced by different manufacturers that use the same hardware mechanisms and operating systems.

(b) the IGOSS MOT proposed for registration was derived from the base implementation by changes that are within the scope of accepted software maintenance practices. Arguments along this line should be included, in writing, with the application.

(c) The PCTR and SCTR for the IGOSS MOT are either the same as the base implementation or, if there are minor differences, these differences are justified as being within the scope of accepted software maintenance practices.

(d) The base implementation was assessed by an approved and registered laboratory and is registered with the Agent of the IGOSS Panel*.

A derived implementation may lose its registration if it is challenged successfully (see B.6.2), upon expiration of the Registration of the base implementation, or when Registration is revoked by the MOT supplier.

The Agent Of the IGOSS Panel ${ }^{*}$ will not perform on-line assessments of derived implementations. The Agent of the IGOSS Panel* will review the application for completeness and plausibility of information. The CSL is the final authority on whether, and in what circumstances, registration shall occur.

Applications which are acceptable to the Agent of the IGOSS Panel $\left.\right|^{*}$ are entered in the register of IGOSS MOTs under the same categorization as the base implementation, (i.e., Registered or Provisionally Registered) and with the same constraints Registration is based on the expiration of the Registered Abstract Test Suite, Provisional Registration expires at the same time as that of the base implementation. The MOT supplier will be notified when registration is approved. The register will be published periodically, and will be available on request from the Agent of the IGOSS Panel*.

\section{B.5.4. Additional Guidance for MOT Registrations}


One of the most significant activities in the IGOSS program is that of placing products on the IGOSS register. This requires that there be a method by which the products can be tested for conformance to the relevant standard(s). This testing is realized by a protocol specific MOT (Means Of Testing) that is also tested and placed on the IGOSS register. The purpose of this section is to provide some level of guidance for the MOT registration other than Base.

The IGOSS program provides two types of MOT registration; base and derived. The "base" registration is the registration of a MOT that has been fully tested by an approved accredited laboratory. This testing consists of an extensive evaluation of both the MOT/user interface and Test Execution. As part of the base registration the MOT vendor is also required to provide a "Baseline IUT PCTR", which is a Test Report, generated from the execution of the MOT at the Vendor's site against a Reference Implementation or Known Sample. This test report is used to verify that any MOT derived from this base MOT is able to execute the test at least as well as the base MOT.

A derived MOT registration is the registration of a MOT that is a modification of a "base" registered MOT.

\section{B.5.4.1 Definitions}

Test Engine: That portion of the MOT that executes the test and records the actions of the IUT via PDUs exchanged with the SUT.

Virtual Machine: The environment within which the Test Engine operates which consists of the hardware platform and operating system.

User Interface: That portion of the MOT that provides the test engineer the capability to interact with and manipulate the Test Engine and other MOT functions. The User Interface permits the test engineer to perform ETS parameterization, execute the ETS, and analyze the log files. It may also provide for test case evaluations, verdicts, log file manipulation, or any other useful activity.

Connectivity: The connectivity identifies the protocols that connect the Test Engine to the IUT. Connectivity is the abstract specification of the supporting stack in terms of protocols.

Underlying Stack: The underlying stack is the specific product(s) that provide the connectivity. The Underlying Stack is specified in terms of the software and/or hardware products that provide the connectivity.

Baseline IUT: The Baseline IUT is a Reference Implementation or Known Sample that is easily accessible to the MOT vendor for purposes of exercising the MOT at the 
"factory". The Baseline IUT must be capable of exercising the entire ETS, but does not have to be capable of passing each test.

In-House Testing: Testing that the MOT vendor conducts against the "Baseline IUT" at a location convenient to the vendor other than an approved laboratory.

Baseline IUT Test Report: The Baseline IUT Test Report is generated by the In-House testing campaign and provides a record of the MOT's behavior relative to the selected Baseline IUT.

Registrations Relative to the Virtual Machine: Changes to only the operating system (e.g., DOS to UNIX), system calls, or the platform are classified as derived Registrations.

\section{B.5.4.2 Generic Guidance}

This section presents different type of guidance depending upon examples of modification to the base MOT

(0) Derived registrations will be allowed for hardware (only) platform changes with conclusive evidence that the Test Engine remains unchanged due to being isolated from the platform architecture by the operating system. This includes both architecture changes and hardware platform clones.

Example:

MOT/POSIX/platformX ==> MOT/POSIX/platformY

MOT/OpSys 1/platformX $==>$ MOT/OpSys 1/platformY

MOT/OPSys $1 /$ platform ==> MOT/OPSys $1 /$ platform clone

(1) Testing Requirement: Full In-House Test (i.e., all ATCs)

Derived registrations will be allowed, without testing, for hardware platform changes if there is conclusive evidence presented that the Test Engine remains unchanged.

Example:

MOT/OpSys/platformX $==>$ MOT/OpSys/platform X'

MOT/OpSys/platformX ==> MOT/OpSys/platformY

(2) Testing Requirement: Full In-House Test (i.e., all ATCs)

Derived registrations will be considered if there is conclusive evidence presented by the applicant that the modifications made in the operating system were transparent to the Test Engine.

Example: 


\section{MOT/OpSysX/platform ==> MOT/OpSysX'/platform}

(3) Testing Requirement: Full In-House Test (i.e., all ATCs)

Changing the virtual machine by altering both the hardware and the software is also acceptable for a Derived registration and requires the same level of MOT testing as an individual change.

Example:

MOT/OpSys1/platformX ==> MOT/OpSys2/platformY

(4) Testing Requirement: Full In-House Test (i.e., all ATCs)

Registrations Relative to Connectivity: Changes in connectivity are allowed as a Derived Registration if there is conclusive evidence presented by the applicant that the modifications made in the operating system were transparent to the Test Engine. The connectivity change must also result in a legitimate IGOSS configuration. The key factor to this registration is the MOT's continued ability to interoperate with the IUT.

Example:

MOT/CLNP/LAN $==>$ MOT/CLNP/X.25

(5) Testing Requirement: Full In-House Test (i.e., all ATCs)

Registrations Relative to Underlying Stack Interface Changes: Derived Registrations will also be allowed where the Underlying Stack is changed, but the protocol connectivity remains the same.

Example:

MOT/CLNP-product X/LAN ==> MOT/CLNP-product Y/LAN

(6) Testing Requirement: Full In-House Test (i.e., all ATCs)

MOT Registrations Relative to User Interface Changes - Because the Test Engine is insulated from most User Interface changes, it is possible to Derived Register a MOT derived from a conformant base MOT given that the Test Engine is unchanged.

Example:

User Interface1/MOT ==> User Interface2/MOT

(7) Testing Requirement: Full In-House Test (i.e., all ATCs) 
MOT Registration Relative to Test Case Correction - A MOT can be Derived Registered based on a Base MOT when one or more test cases are corrected, given that the rest of the Test Engine is unaffected.

Example:

$$
\text { MOT(V1)/CLNP/LAN ==> MOT(V2)/CLNP/LAN }
$$

(8) Testing Requirement: Full In-House Test plus detailed log files for the corrected ETC(s)

MOT Registration Relative to Test Case Addition: MOT can be Derived Registered from a Base MOT when one, or more, test cases are added given that the rest of the Test Engine is unaffected and the new ETCs come from the registered ATS and are correctly implemented.

Example:

$$
\text { MOT(V1)/CLNP/LAN ==> MOT(V2)/CLNP/LAN }
$$

(9) Testing Requirement: Full In-House Test plus detailed log files for the modified ETC(s)

MOT Registration Relative to Test Case Deletion: A MOT can be Derived Registered from a Base MOT if the Test Engine is altered to affect the removal of the test case given that the rest of the Test Engine is unaffected.

Example:

$$
\text { MOT(V1)/CLNP/LAN ==> MOT(V2)/CLNP/LAN }
$$

(10) Testing Requirement: Full In-House Test

MOT Registrations Relative to Version Changes: Because MOT version changes are often due to modifications unrelated to the Test Engine, Derived Registrations will be allowed for a MOT derived from a Base MOT given that the changes do not affect the Test Engine.

Example:

MOT(V1)/CLNP/LAN $==>$ MOT(V2)/CLNP/LAN 
(11) Testing Requirement: Full In-House Derived Testing

MOT Registrations Relative to Added whole ATSs: A Derived Registration will be allowed for any MOT that adds at least one new ATS to its repertoire given that the changes do not affect the Test Engine. The testing will typically be a subset of the original Base Registration selected to test those aspects of the MOT that are new (e.g., documentation and ETC analysis).

Example:

$$
\text { MOT(ATS } x) / C L N P / L A N==>\text { MOT(ATS }+ \text { ATSy)/CLNP/LAN }
$$

\section{B.5.5 Mutual Recognition Arrangements}

A Means of Testing registered by another authority may be registered with the Agent of the IGOSS Panel* if:

(a) A Memorandum of Understanding exists between the Agent of the IGOSS Panel $^{\star}$ and the registering authority, recognizing each other's assessment and registration procedures.

(b) Specific application is made to the Agent of the IGOSS Panel* by the MOT supplier. (Automatic concatenation of registers will not occur).

(c) The candidate MOT for IGOSS registration passes the Agent of the IGOSS Panel ${ }^{*}$ static evaluation.

(d) The candidate MOT has not been removed from the Agent of the IGOSS Panel* register as a result of a successful challenge which has not been resolved by the primary assessing authority.

If the above conditions are met, the candidate MOT will be registered with the same expiration date as for the primary assessing authority, or until the expiration date of the associated Abstract Test Suite, whichever is earlier.

If an MOT which is registered under the provisions of a Mutual Recognition Arrangement is removed from the primary authority's register then it shall be removed from the IGOSS register.

The Agent of the IGOSS Panel* will not perform on-line assessments of implementations assessed by a mutually recognized authority. The Agent of the IGOSS Panel* will review the application for completeness and plausibility of information. The Agent of the IGOSS Panel is the final authority on whether, and in what circumstances, registration shall occur.

Applications which are acceptable to the Agent of the IGOSS Panel ${ }^{*}$ are entered in the register of IGOSS conformant MOTs under the same categorization as the primary 
registration (i.e., Registered or Provisionally Registered) and with the same constraints - Registration is based on the expiration of the Registered Abstract Test Suite, Provisional Registration expires at the same time as that of the primary registration. The MOT supplier will be notified when registration is approved. The register will be published periodically, and will be available on request from the Agent of the IGOSS Panel*.

\section{B.6. Dispute Policy}

Any Registered implementation may be challenged by any interested party through the Agent of the IGOSS Panel ${ }^{*}$. The challenger will pay a challenge fee to the Agent of the IGOSS Panel ${ }^{*}$ and will submit a challenge request which:

(a) identifies the implementation being challenged,

(b) names one or more tests from the Executable Test Suite,

(c) describes the way in which the test(s) are invalid.

\section{B.6.1 Challenges to Base Implementations}

The Agent of the IGOSS Panel* will send the challenge to the MOT supplier asking for comment. If the MOT supplier does not adequately address the problem within 30 days then the MOT is statically reassessed according to the criteria in B.3.2 of this Annex. If the MOT no longer meets the criteria for the category in which it is registered then the MOT's registration is revoked. The MOT supplier may subsequently demonstrate correct operation of the test and it will be reinstated.

\section{B.6.2 Challenges to Derived Implementations}

The Agent of the IGOSS Panel* will send the challenge to the MOT supplier asking for comment. If within 30 days the supplier is unable to demonstrate correct operation of the test(s), the derived implementation will be marked as Provisionally Registered with an expiration date a further 90 days hence. The rules for Provisional Registration of a base implementation apply thereafter.

\section{B.6.3 Challenges to 'Mutual Recognition Arrangement' Implementations}

The Agent of the IGOSS Panel ${ }^{*}$ will send the challenge to the primary registering authority asking for comment. If not already so categorized then the MOT will be marked as Provisionally Registered with an expiration date 90 days hence. If the primary registering authority is subsequently satisfied that the test(s) operate correctly then the former categorization is reinstated. 


\section{B.6.4 Abstract Test Suite Problem Resolution}

In the long term it is hoped that Abstract Test Suites and related test case interpretation issues will be authorized by the relevant ISO working groups. However a more immediately responsive forum, technically grounded and sufficiently democratic, is the OSE Implementors' Workshop, through the Conformance Testing SIG and the appropriate technical SIGs. The GOSIP Testing Program will accept rulings on test case interpretation from this forum.

\section{B.6.5 Reference Implementation Problem Resolution}

For each IGOSS protocol the Agent of the IGOSS Panel ${ }^{*}$ maintains a Reference Implementation, or identifies a 'known' implementation. In either case it is unlikely that this implementation will be completely bug-free. Fortunately, for the purpose of MOT assessment it is not either necessary, or even desirable, to maintain a perfect implementation. It is however, necessary for the Agent of the IGOSS Panel ${ }^{*}$ to maintain a catalogue of the known problems with the implementation. It is appropriate that during assessment, an MOT is able to discover these problems and mark the appropriate tests with a 'Fail' or 'Inconclusive' Verdict.

Consequently, any new problems found with the known implementation will, after corroboration, be added to the catalogue of errors. Any fixes will be deleted from the catalogue. 


\section{ANNEX C: HOW TO ACCESS THE REGISTERS}

\section{C.1 On-Line Registers}

The United States Government Open Systems Interconnection Profile (GOSIP) Testing Program was defined to permit Federal Agencies to substantiate claims of GOSIP compliance. As a result of this program, Federal Agencies can ensure that GOSIP compliant products are effectively tested for conformance to the Open Systems Interconnection (OSI) standards and for interoperability with other OSI implementations. The IGOSS Testing program has been built up from the GOSIP Testing program.

This document produced by the National Institute of Standards and Technology (NIST) entitled "IGOSS Testing Framework" establishes the framework by which IGOSS Acquisition Authorities can ensure that procured IGOSS products are compliant with the IGOSS specifications. The vehicle by which this determination can be made is a set of registers. These include registers for Test Suites, Test Systems (Means of Testing), Conformance Testing Laboratories, and Interoperability Testing Services.

The U.S. GOSIP/IGOSS Register Database is an online database facility developed by NIST and JITC. It provides up-to-date reference information for the following list of registers:

1. IGOSS PICS Proforma

2. IGOSS Abstract Test Suites (ATS).

3. Assessed Means of Testing (MOT).

4. NVLAP or SCC Accreditated Test Laboratories.

5. Conformance Tested IGOSS Products.

6. Interoperability Test Suites (ITS) for OSI Products.

7. Reference Entities for Means of Testing Assessment(s).

8. Interoperability Test and Registration Services.

9. Approved IGOSS MOT Validation Laboratories.

To access the GOSIP/IGOSS registers, follow these instructions:

\section{C.1.2 To reach the Database}

Dial in on a $1200 / 2400$ baud analog modem (Bit/Char: 8 , Stop Bits: 1, Parity: None) to (602) 538-5233. There are actually four lines on rotary to ensure access. Once you are connected, you will see the login prompt. Respond with the user name "jitc1" in the lower case only. No password is needed. You will be asked for your name, and then asked to designate the terminal emulation you use. You will then sequence through a series of menu screens that allow you to access and read GOSIP/IGOSS register data and generate ASCII text report files to download to your computer. 
JITC has tested dial-in access and file downloading using Procomm Plus and Kermit on a PC.

You can also access the Database through the Internet. The host name is huachucajitcosi.army.mil and the Internet protocol address is 138.27.7.2. Two separate logins are required, both with user name "jitc1" and no password. The first login activates a shell script that immediately dials out to connect to the Database. If the outgoing line is busy, the user will be notified and then automatically logged out.

All data access functions are supported over the Internet, but file downloading is not supported.

\section{C.1.2 For server access to downloadable files:}

The GOSIP/IGOSS register files for all GOSIP/IGOSS registers, along with the product and MOT registration application forms, are available on a host computer which supports faster downloading. The files are available in WordPerfect Version 5.1 and ASCII text formats.

You can access the downloadable files on this host through dial-in modem or through the Internet.

You can dial in on a 2400 baud analog modem (8-1-N-2400) to (602) 538-5004/4323, or on a 9600 baud digital modem (8-1-N-9600) to (602) 538-5034/5036. Once you have accessed the host, hit "Enter." This will bring up an alert screen, and ask for your login name. At the login prompt, type "jitc1" in lower case or upper case. Again, no password is needed. A menu will be displayed. You may read a page of instructions, review register files, and download register files.

If you decide to download files, you have to access Kermit. The instructions will tell you to select the download choice, and at the prompt to hit "PgDn." That gives you several selections, and you should choose Kermit.

To download GOSIP/IGOSS register files from the host over the Internet, ftp to Internet Protocol address 138.27.8.2. Login as "anonymous" and give you user name as the password. Change directories to the GOSIP directory (e.g., cd gosip). Then list the files (e.g., Is). For the Wordperfect files, you need to type "binary." Then type in "quote site io_mode." Then you can "get" the files. For ASCIl files, you can omit the "binary" and "quote site io_mode" commands.

In order to improve the service, JITC has established an additional host for Internet downloading of GOSIP/IGOSS register files. For the new host, the procedure is to ftp to huachuca-jitcosi.army.mil (138.27.7.2), login as "anonymous" and a text file of 
instructions and subdirectories for GOSIP/IGOSS register files and registration forms. Both ASCll text files and WordPerfect files are included. Use "binary" when transferring WordPerfect files.

JITC has established a new telephone number for GOSIP/IGOSS registration support, including assistance in accessing the register. The number to call is (602) 538-4351.

\section{C.2 Validated Products List}

The Validated Products List (VPL) is published quarterly and currently distributed by the National Technical Information Service (NTIS). For order please contact:

National Technical Information Service

U.S. Department of Commerce

5285 Port Royal Road

Springfield, VA 22161

for subscriptions call: (703) 487-4630

for individual copies: (703) 487-4650

The entries in the Validated Products List may be accessed on the Internet using the following instructions:

type: ftp speckle.ncsl.nist.gov

(internet address 129.6.59.2)

Login as user ftp

type your e-mail address as the password

type: cd vpl or cd pub/vpl

type: binary

The directory contains the full VPL, type Is for complete list. 
This Page is intentionally blank 


\section{ANNEX D: ADVICE TO PROCUREMENT AUTHORITY}

\section{D.1 Introduction and Statement of the Problem}

The IGOSS Testing Program has been set up from the U.S. GOSIP Testing Program. As such Acquisition Authorities inherit the same type of procurement strategy. This Annex refers to the U.S. GOSIP Testing Program as an example.

Following the publication of FIPS 146 [NIST 17], it became mandatory for Federal Agencies to procure networking products based on the Open Systems Interconnection specifications as from August 1990. With the publication of FIPS 146-1 [NIST 18], the required functionality were expanded, from FTAM and X.400 over LAN and WAN technologies, to embrace Virtual Terminal applications and ISDN. With these complex and expensive technologies, it is not enough for vendors to make unsubstantiated claims of compliance. It is necessary to have a demonstration of compliance.

This Annex discusses how the IGOSS Testing Policy can be used to assist in Federal procurements, and what extra steps an Acquisition Authority must take to secure quality products.

\section{D.2 The IGOSS Registers}

The visible result of the IGOSS Testing Policy is a database of Registers listing the various elements of the program. The complete list of Registers is:

1) IGOSS PICS Proformae

2) IGOSS Abstract Test Suites

3) Interoperability Test Suites

4) Validated Means of Testing

5) NVLAP or SCC Accredited Conformance Testing Laboratories

6) IGOSS Reference/"Sample" Implementations

7) Conformance Tested IGOSS Products

8) Interoperability Testing Services

9) MOT Validation Laboratories

The single register of most use for procurement purposes is the Register of Conformance Tested IGOSS Products. Products successfully tested in a NVLAP or SCC Accredited Laboratory are entered onto this Register. The next most useful register is the Interoperability Testing Services Register, which is a list of services approved to conduct and advertise pairwise interoperability testing between IGOSS products. 


\section{D.2.1 The Register of Conformance Tested IGOSS Products}

IGOSS products which have been successfully tested by an accredited test laboratory using a registered Means of Testing, including registered Abstract Test Suites, may be added to the register of Conformance Tested IGOSS Products. The goal of conformance testing is to verify product/protocol compliance to the relevant standard. The register contains a list of products that have successfully passed conformance testing (i.e., not demonstrated any instance of non-compliance during the test campaign). Conformance testing is not a $100 \%$ warranty that the product you are purchasing is compliant. It does indicate that a minimum amount of testing using globally accepted test specification has been applied successfully to the products registered.

Testing of OSI End Systems has been modularized to separate supporting network connectivity, intermediate layers, and applications, and the structure of the IGOSS Products Register reflects these separations. There are separate categories such as:

WAN Products (X.25, ISDN, Frame-Relay)

LAN Products $(802.3,802.4,802.5)$

CLNP Products (Intermediate Systems)

Transport Products (TP2, TP0, and TP4/CLNP End Systems)

Session Products

X.400 Products (Message Handling Systems) 1984 and 1988

FTAM Products (File Transfer Applications)

X.500 Products (Directory Service Application)

MMS Products (Manufacturing Message Specification Application)

Network Management Products

For End Systems procurement, your concern is with the X.400, FTAM, and Virtual Terminal categories. The Session, Transport, LAN and WAN categories provide supporting connectivity for these applications. For Intermediate Systems/Gateway procurements, your concern is with the CLNP products category. LAN and WAN categories also provide supporting connectivity to Intermediate Systems (or "Routers"). Entries in each category are as follows:

- $\quad$ The name of the Register category, e.g. X.400 Products.

- $\quad$ Supplier Name and Address.

- $\quad$ Contact name, with Phone and Fax Numbers.

- GOSIP Product Name, Release and Date, e.g. ACME Software X.400 Messager, Version 27.92, October 1992. 
Hardware and Operating System Platform, e.g. ACME Hardware 2900 Series, ACMIX Version 14.7.

Base/Derived, i.e. whether the product was fully tested on this platform (Base), or ported from another H/W O/S platform (Derived).

Connectivity, i.e. in the case of an X.400 application, the associated registered Session platform.

Protocols and Profiles, e.g. CCITT X.400 1984 Series, P1, P2 and RTS.

Date Registered.

Type of Registration, i.e. Provisional or Full. Depends on the state of maturity of the Abstract Test Suite and Means of Testing. Full Registration only occurs after the Abstract Test Suite has reached International Standard, and test tool coverage is at the $95 \%+$ level. For Provisional Registration, coverage is at $60 \%+$ or $80 \%+$. In this context, coverage is only a measure of the minimum requirements for test tool vis-à-vis the test suite (i.e., if a test tool implements properly 69 test cases out of 100 test cases in the registered abstract test suite, the test tool is said to cover at $69 \%$ ). There is no clear relationship between this measure and how much of the protocol is tested.

Conformance Lab. Used, e.g. the ACME Conformance Testing Center (Should be on the list of NVLAP or SCC Accredited Labs).

Some products in the register have been grandfathered from one GOSIP/IGOSS version to another. Grandfathering is the act of granting GOSIP/IGOSS Version N+1 conformance testing registration to a GOSIP/IGOSS Version $N$ registered product without requiring conformance testing. Such products are clearly identified in the register.

For each product registered the vendor/bidder has the ability to provide you with complementary information about the product functionality. A set a documents was employed during the test campaigns and describe completely each protocol layer capability. These documents are the Protocol Implementation Conformance Statements (PICS) and Protocol Implementation eXtra Information for Testing (PIXIT). The PICS is a statement made by the supplier of an OSI implementation, or system, stating which capabilities and options have been implemented, for a given OSI protocol. The PIXIT is a statement made by a supplier or implementor of an IUT which contains or references all of the information (in addition to that given in the PICS) related to the IUT and its testing environment, which will enable the test laboratory to run an appropriate test suite against the IUT. These documents can be used by technical experts to assess how the products match the actual requirements. These documents 
can be easily used to verify that the product proposed matched the actual product tested and registered.

\section{D.2.2 The Register of Interoperability Testing Services}

This register contains the addresses and point of contact of the registered interoperability testing services:

Each of these organizations maintains a register of interoperability tested products, which have been tested against the Interoperability Test Suites.

Each organization uses different procedures. You are advised to get a copy of the relevant documentation and assess whether the organization matches you quality requirements. For each interoperability test campaign, each organization should:

1) Conduct a static analysis phase which involves the selection of a common subset of the IGOSS tests including all of the mandatory tests and all the relevant optional tests resulting from the implementation of the same optional functionality;

2) Conduct a dynamic analysis phase in which both IGOSS product suppliers are in agreement concerning the outcome of each test;

3) Issue a test report which identifies each IGOSS product supplier, describes the product including the supporting stack of protocols, and provides the list of the tests selected and executed with a verdict for each test. The verdict may be 'pass' or 'fail'. Any fail verdict must be accompanied by an explanation outlining the cause of failure. Any non selected test case must be accompanied by an explanation outlining the rational for de-selection.

You may require a copy of the interoperability testing test report and verify that the product has successfully demonstrated all the claimed functionality.

\section{D.3. Procurement Strategy}

The following text is recommended:

"Any computer networks and communications equipment, systems and services offered in response to the specific functional requirements in this solicitation must provide the associated protocols specified in the standards referenced by IGOSS or Industry/Government Open Systems Specification."

However there are some important things to note: 
1) The IGOSS Testing Program specifies communications technology only. other Information Processing concerns are outside its scope.

2) We recognize that many procurements are for larger scale, general purpose ADP (Automated Data Processing) acquisitions, involving a mixture of different specification. There are very many ways of combining these specification. There are very many ways of combining the protocol profiles within the IGOSS. Business Unit functional requirements are what determine the mix.

The general wording, above, explicitly allows the possibility of acquiring individual slices through the IGOSS profile. However, this requires that the solicitation be augmented with a detailed schedule of actual IGOSS applications and connectivity which are sought.

\section{D.3.1 The Schedule}

For instance if the Acquisition Authority requirement is for Electronic Message Handling Service, then the X.400 application is what you need to acquire. The need may be for a service attached to a public network, or for a private messaging service. The alternative full stacks for these options are:

Public Messaging Service attachment:

X.400/Session/Transport Class 0/X.25

Private Messaging Service:

X.400/Session/Transport Class 4/CLNP/LAN or WAN.

Where WAN $=X .25$; $L A N=802.3,802.4$, or 802.5 .

More likely, you will need to acquire both TPO and TP4 solutions to encompass LAN/WAN inter networking situations. If your solicitation includes at least the Applications and the LAN and/or WAN networks, then this can be used in conjunction with the appropriate general statement above, to define the relevant portions of IGOSS to be provided.

\section{D.3.2 Specification of Options}

In some cases the base (ISO/CCITT) standards provide optional functions which are not resolved in the Stable Implementation Agreements [NIST 1] or in FIPS 146-2. Any such options required by an Acquisition Authority must be explicitly called out and verified with a supplier, who may or may not have chosen to implement such options. [See also D.4.5].

\section{D.3.3 Assurance of Troubleshooting/Interoperability Support}

The question of Interoperability Assurance prior to acquisition and installation is covered in section D.4.4 below. However it should be noted that even a combination of 
conformance and interoperability testing cannot give guarantees of complete interworking. Inevitably, problems will develop after installation, both within the scope of the acquisition and with other IGOSS systems. For this reason it is important to secure cooperative maintenance arrangements with IGOSS suppliers, such that each supplier associated with an interoperability problem is required to participate in its resolution. This can easily be arranged by contract, within the scope of an acquisition but there are several possibilities:

1) (Intra Business Unit, Intra Procurement) - Interoperability problem between suppliers under the scope of a single acquisition: as above.

2) (Intra Business Unit) - Interoperability problem between suppliers of any two systems within an Business Unit: therefore any supplier of IGOSS products to an Business Unit, irrespective of when the acquisition(s) occurred, must commit to a cooperative maintenance arrangement.

3) (Inter Business Unit) - Interoperability problem between suppliers of systems to different Business Units: therefore any pair of suppliers of IGOSS products to Business Units involved in the problem, must be prepared to assist in problem resolution. For this reason every acquisition must require participation in problem resolution on the part of any involved supplier who provides a product to any Business Unit at any time.

4) (Business Unit - Rest of the World) - Interoperability problem between suppliers of systems to an Business Unit and any other organization: recourse to the other supplier is probably limited, except insofar as that supplier may have entries on IGOSS conformance or interoperability registers which could be jeopardized by non-cooperation.

In summary, the best assurance of government-wide interoperability can be gained by every Business Unit requiring that all IGOSS suppliers commit to participating in the resolution of any interoperability problem in which their system is involved. This can be greatly facilitated by the encouragement of all IGOSS suppliers to participate in preprocurement Interoperability Testing; and in addition, that post-procurement Interoperability Analysis services be widely recognized and used.

\section{D.4 Acquisition Testing Strategy}

\section{D.4.1 Introductory Discussion}

Systems offered in response to a solicitation which includes IGOSS functionality must be demonstrated, or have been demonstrated, to be IGOSS compliant. The only way to make this determination is by successful conformance testing in a NVLAP or SCC accredited laboratory, leading to entry on the Conformance Tested IGOSS Products Register. This requirement should be applied on a protocol by protocol basis. There 
are some protocols for which conformance testing infrastructure (Abstract Test Suites and Means of Testing) does not yet exist - in particular, the Virtual Terminal application. A list of validated IGOSS products can be found in the NIST Validated Products List [5] produced by the NIST Computer Systems Laboratory and published quarterly (December, March, June, September). However the most current list of products may be found in the GOSIP/IGOSS Database Register. This register contains only products that have been fully validated. It does not contain products available but not tested, nor products under test.

In certain technical areas, the GOSIP/IGOSS Products Register, as it stands right now, may not be well populated with tested applications. In order to achieve an acceptable mix of competitive bids an Acquisition Authority may be compelled to solicit and review bids for products which are not yet tested.

Once a requirement for functionality exists, one or more of the following problem conditions may exist: 1) no products, or only a very limited number of products are available; 2) conformance tests are not available; 3) a very limited number or no products have passed conformance testing. Test availability is defined as conformance tests being available within at least one third party Accredited Conformance Test Laboratory.

The following is assumed: if products are available and a test is available, then vendors are at least attempting to pass the conformance test; if no or only a very limited number of validated products are available, then the Acquisition Authority will want to give vendors time to successfully validate each product.

\section{D.4.2 Validation Options}

The following definitions apply: PV is Prior Validation, UT is Under Test (formerly Prior Validation Testing), DV is Delayed Validation, DD is Delayed Delivery.

\begin{tabular}{|l|l|l|l|l|l|l|l|l|l|}
\hline Products Available & Y & Y & Y & Y & V & V & V & N & N \\
\hline Test Available & Y & Y & Y & N & Y & Y & N & Y & N \\
\hline Products Validated & Y & V & N & & V & N & & & \\
\hline Paragraph & PV & UT & UT & DV & UT & UT & DV & DD & DD \\
\hline
\end{tabular}

Where $\mathrm{Y}=\mathrm{Yes} ; \mathrm{V}=$ Very limited Number; $\mathrm{N}=\mathrm{NO}$

\section{Prior Validation (PV)}

"The offeror shall certify in the offer that implementations of the appropriate protocol, including applicable options, offered in response to this document have been previously validated". 
Note that Prior Validation is demonstrated by a product's inclusion in the IGOSS Product Register. There are currently NO certificates issued, nor are there Validation Summary Reports (VSR) available for IGOSS Products.

\section{Under Test (UT)}

"The offeror shall agree to correct all implementation non conformance from the applicable protocol. All areas of non conformance must be corrected within [a specific period of time i.e. 3 to 6 months] from the date of contract award. If an interpretation of IGOSS is required that will invoke the procedures set forth such these in FIPS 29-2, such a request for interpretation shall be made within 30 calendar days after contract award. Any corrections that are required as a result of decisions made under the relevant procedures shall be completed within (a specific time i.e. 3-6 months of the date of formal notification to the contractor of the approval of the interpretation. Proof of correction in either case shall be inclusion on the IGOSS Products Register. Failure to make the required corrections within the time limits set forth above shall be deemed a failure to deliver required software. The liquidated damages as specified for failure to deliver the operating system or other software shall apply."

\section{Delayed Validation (DV)}

"The offeror shall certify in the offer that the implementation of the protocol, including applicable options offered in response to this document will be submitted for validation and validated within 6 months after conformance testing services are available. Unless specified elsewhere, proof of submission for validation shall be in the form of a letter scheduling the test campaign, issued by an Accredited Conformance Testing Laboratory. Once the product is Under Test the conditions of that option apply."

You can estimate when validation tests will become available by reviewing the Register of Abstract Test Suites and the Register of Validated Means of Testing.

\section{Delayed Delivery (DD)}

If none or a very limited number of products are available then the Acquisition Authority should consider postponing the delivery of the requirement until either the conditions of "Under Test" or "Delayed Validation" exist.

\section{D.4.3 Adding IGOSS Capability to Existing Installations}

All of the foregoing applies to the procurement and testing of combinations of hardware and software, representing new acquisitions. The case of adding IGOSS capability to existing installations is broadly the same, except in this case a sole-source justification may also be required. Other than this, the choice of Prior Validation, Under test, Delayed Validation and Delayed Delivery is as above.

\section{D.4.4 Interoperability Testing as Extra Insurance for Product Acceptability}


Conformance testing is good because it helps to demonstrate a product's conformance to the published standards; however, on its own it does not provide a guarantee of interoperability between conformance tested products. Interoperability testing is good because it provides a method for IGOSS product implementations to achieve interworking; however on its own, without conformance testing, the patches applied to enable product interworking may cause drift away from the standards, thereby making it difficult for subsequent suppliers to attach their conformant implementations to the installed base. Therefore the optimal long term goal is to achieve conformant interoperability. Although interoperability testing is not mandated in the IGOSS Testing Program, an Acquisition Authority should require it.

\section{D.4.5 Extra Testing Possibilities}

\section{D.4.5.1 Security Features}

At present there is no testing program in place for any of the security options within IGOSS. An Acquisition Authority wishing to acquire these options must be prepared to specify its own conformance and acceptance tests.

\section{D.4.5.2 Performance Requirements}

At this early stage of the deployment of IGOSS, there is insufficient installed base to develop meaningful performance statistics. Any Acquisition Authority wishing to levy performance requirements on IGOSS procurements must first develop the performance criteria and then arrange to have all applicable products exercised in order to apply the criteria. It is unlikely that very many implementations will meet stringent performance criteria today. Therefore, the IGOSS Testing Program recommends that an Acquisition Authority develop its own performance criteria, or encourage the development of generic performance criteria, and gain experience in measuring implementations for later use in requirements setting.

\section{D.4.6 A Note on Diagnostic Equipment}

In order to troubleshoot IGOSS problems it may be necessary for Acquisition Authorities to acquire special-purpose diagnostic equipment. Such analyzers have long existed in the form of line monitors, LAN monitors and X.25 packet decoders. However, one can expect the burgeoning OSI market to precipitate the need for, and supply of, multi-layer OSI protocol decoders, spanning one or more layers of protocol from LAN to Application. There is today no requirement in the IGOSS Testing Program to test and register this equipment. When procuring such an analyzer an Acquisition Authority should specify tests of its diagnostic capability for each protocol for which support is claimed. Presenting the results of a decoded Interoperability test may be one way to do this. Presenting the results of a decoded Conformance Test is probably better, since all Protocol Data Unit fields are spelled out in each Conformance Test. 


\section{D.4.7 Holes in the Testing Infrastructure}

There are some cases where an Abstract Test Suite and/or a Means of Testing do not exist for particular IGOSS protocols. The three Validation options given above are inadequate to deal with these cases. You can determine what these cases are by examining the Register of Abstract Test Suites and the Register of Validated Means of Testing. It is possible that, for instance, Virtual Terminal products may become available and the means for validating them be indefinitely delayed. Therefore even employment of "Delayed Validation" wording will not yield Validated Products by the time of delivery to the Acquisition Authority. If you have available the qualified technical resource, you may develop a specific acceptance testing program.

\section{D.5 Sample Text}

This section is for information only and provides guidance on how to describe IGOSS testing requirements.

[PROCUREMENT-NAME] - Statement of Work:

General Requirements: "... requires that all computer network and data communications systems and services procured comply with the requirements of the IGOSS [REF] and the IGOSS Testing Framework [REF]."

1. "The technical objective standard for [PROCUREMENT-NAME] is IGOSS Version 1.0. Because Abstract Test Suites (ATS) may not be available for IGOSS Version 1.0 testing in time to meet the [PROCUREMENT-NAME] acquisition schedule, the Contractor shall implement the network using equipment that appears on the NIST Register of GOSIP compliant products, tested at least to GOSIP Version 2.0. At such time IGOSS 1.0 ATS are available, the Contractor shall retest applicable products for compliance with IGOSS 1.0. Should the equipment fail IGOSS 1.0 testing, the Contractor shall, at his cost, take whatever remedial actions are necessary to bring the equipment to IGOSS 1.0 standards and have it retested. Alternatively, the Contractor may elect to replace that equipment, at his cost, with another vendor's equipment that does meet IGOSS 1.0 standards."

2. "The Contractor may propose use of untested products having GOSIP 2.0 functionality to meet a specified [PROCUREMENT-NAME] requirement provided testing is completed by the time of submission of Best and Final Offers (BAFO). Should testing not be completed by the time of submission of BAFO, the Contractor shall offer a substitute piece of equipment which has been GOSIP 2.0 registered."

3. "In the event there are no IGOSS certified products available for substitution, or there is no applicable ATS for the item, or if there are no IGOSS compliant 
products available in industry to meet the specified requirement, then and only then may a non-IGOSS compliant item be offered. If this occurs, the item shall be identified as a IGOSS compliance deficiency in the migration plan required by [MIGRATION-TEXT-REF]."

4. "Should a registered IGOSS product suitable for an application identified as a IGOSS deficiency in the IGOSS migration plan become available in the interim, the Contractor shall have 180 days to either replace his non-equipment with the available certified equipment, or make his equipment compliant and complete certification testing. The Acquisition Authority will discontinue paying recurring charges on any non-certified equipment being used in the network 180 days after certified equipment that meets the functional requirements becomes available on the market."

\section{Definition:}

IGOSS Compliant: "Is defined as the Contractor implementing IGOSS protocols in accordance with IGOSS and providing equipment for use in [PROCUREMENT-NAME] with IGOSS functionality that adheres to the provisions of the IGOSS Testing and Registration Program as described in the IGOSS Testing Framework Technical Report [REF] and that appears on the NIST Register of Conformance Tested GOSIP/IGOSS Products."

Note: A GOSIP V2.0 Compliant product may well be IGOSS Compliant. Indeed, there are protocols for which IGOSS may not add any requirement/functionality or any testing requirement. 
This Page is intentionally blank 


\section{ANNEX E: BIBLIOGRAPHY AND REFERENCES}

\section{American National Standards Institute}

1. Integrated Services Digital Network - Basic Access Interface for Use on Metallic Loops for Application on the Network Side of the NT-Layer 1 Specification, ANS T1.601-1988.

2. Integrated Services Digital Network - Basic Access Interface at S and T Reference Points - Layer 1 Specification, ANS T1.605-1988.

3. Carrier to Customer Installation - DS1 Metallic Interface, ANSI T1. 403-1989.

4. American National Standard Information Retrieval Application - Service Definition and Protocol Specification for Open Systems Interconnection, ANSI Z39.50-1992.

5. Information Processing Systems - Computer graphics - Metafile for the storage and transfer of picture description information, ANSI/ISO 8632.

6. American National Standard - Digital Representation for Communication of Product Definition Data, ANSI Y14.26M-1989.

7. American National Standard - Information Retrieval -Application Service Definition and Protocol Specification for Open Systems Interconnection, ANSI Z39.50.

8. American National Standard for Information Systems - Computer Graphics - X Window System Data Stream Definition, ANSI X3.196-199X.

9. ANS T1.617 - DSS1 - Core Aspects of Frame Protocol for Use with Frame Relay Bearer Service, 1991

10. ANS T1.618 - DSS1 - Signaling Specification for Frame Relay Bearer Service, 1991

11. ANS X3.216 - Structure and Semantics of the Domain Specific Part (DSP) of the OSI Network Service Access Point (NSAP) Address.

12. ANS X3.229 - 199X - Fiber Distributed Data Interface (FDDI) Station Management (SMT). (ANSI X3T9 approved SMT Version 7.2)

\section{Canadian Standards Association}

1. CSA Z243.110.2 Canadian OSI Registration Procedures and Guidelines - Part 2 -Guidelines for Network Service Access Point Addresses for the Data Country Code Format.

\section{Electronic Industries Association}

Interface between Data Terminal Equipment and Data Communication Equipment Employing Serial Binary Data Interchange, EIA-232C.

Institute of Electrical and Electronics Engineers, Inc.

1. Standard for Information Technology -- OSI Application Program Interface - Common ASN.1 Object Management API for X.400 and Directory Services APIs, P1224.

2. Standard for Information Technology -- OSI Application Program Interfaces -- X.400 Based 


\section{Electronic Messaging API, P1224.1.}

3. Standard for Information Technology, Portable Operating System Interface (POSIX) -- Directory Services Application Programming Interface, P1003.17.

4. Draft Standard 802.1B: LAN/MAN Management, P802.1B/D20, January 27, 1992.

5. Draft Supplement to IEEE Std. 802.3: Repeater Management, P802.3.KJD5, December 22, 1991.

The above documents may be obtained from: IEEE Standards Office, 345 East 47th Street, New York, N.Y. 10017. Phone (800) 678-4333.

\section{International Organization for Standardization}

NOTE: Several versions of some of the ISO references that follow may exist. Different versions of the same reference are distinguished by the year of publication. The reader should consult the appropriate section of the Workshop Agreements to determine the applicable version when a date is not provided.

1. Information Processing Systems - Open Systems Interconnection - Basic Reference Model, Ref. No. ISO 7498-1984(E).

2. Information Processing Systems - Data Communications - Use of $X .25$ to provide the OSI Connection Mode Network Service, ISO 8878.

3. Information Processing Systems - Open Systems Interconnection - Network Service Definition, ISO 8348.

4. Information Processing Systems - Open Systems Interconnection - Addendum to the Network Service Definition Covering Connectionless Data Transmission, ISO 8348 Addendum 1.

5. Information Processing Systems - Open Systems Interconnection - Addendum to the Network Service Definition Covering Network Layer Addressing, ISO 8348 Addendum 2.

6. Information Processing Systems - Open Systems Interconnection - Internal Organization of the Network Layer, ISO 8648.

7. Information Processing Systems - Open Systems Interconnection - Protocol for Providing the Connectionless Network Service, ISO 8473.

8. Information Processing Systems - Open Systems Interconnection - Data Communication - X.25 Packet Level Protocol for Data Terminal Equipment, ISO 8208.

9. 7-bit Coded Character Set for Information Processing Interchange, ISO 646, 1973.

10. Information Interchange - Representation of Local Time Differentials, ISO 3307, 1975.

11. Information Processing Systems - Open Systems Interconnection - Working Draft - End System to Intermediate System Routing Exchange Protocol for use in Conjunction with ISO 8473.

12. Information Processing Systems - Open Systems Interconnection - Transport Service Definition, ISO 8072.

13. Information Processing Systems - Open Systems Interconnection - Transport Protocol Specification, ISO 8073. 
14. Information Processing Systems - Open Systems Interconnection - Session Service Definition, ISO 8326.

15. Information Processing Systems - Open Systems Interconnection - Session Protocol Specification, ISO 8327.

16. Information Processing Systems - Open Systems Interconnection - File Transfer, Access and Management Part 1: General Introduction, ISO 8571-1.

17. Information Processing Systems - Open Systems Interconnection - File Transfer, Access and Management Part 2: The Virtual Filestore Definition, ISO 8571-2.

18. Information Processing Systems - Open Systems Interconnection - File Transfer, Access and Management Part 3: File Service Definition, ISO 8571-3.

19. Information Processing Systems - Open Systems Interconnection - File Transfer, Access and Management Part 4: File Protocol Specification, ISO 8571-4.

20. Information Processing Systems - Open Systems Interconnection - Connection-Oriented Presentation Service Definition, ISO 8822.

21. Information Processing Systems - Open Systems Interconnection - Connection-Oriented Presentation Protocol Specification, ISO 8823.

22. Information Processing Systems - Open Systems Interconnection - Service Definition for Association Control Service Element - Part 2: Association Control, ISO 8649.

23. Information Processing Systems - Open Systems Interconnection - Protocol Specification for Association Control Service Element: Association Control, ISO 8650.

24. Information Processing Systems - Open Systems Interconnection - Specification of Abstract Syntax Notation One (ASN.1), ISO 8824.

25. Information Processing Systems - Open Systems Interconnection - Specification of Basic Encoding Rules for Abstract Syntax Notation One (ASN.1), ISO 8825.

26. Information Processing Systems - Data Communications - High-Level Data Link Control Procedures - Description of the X.25 LAPB-compatible DTE Data Link Procedures, ISO 7776.

27. Information Processing Systems - Data Interchange - Structures for the Identification of Organizations, ISO 6523, 1984.

28. Information Processing Systems - Local Area Networks - Part 2: Logical Link Control, ISO $8802 / 2$.

29. Information Processing Systems - Local Area Networks - Part 3: Carrier Sense Multiple Access With Collision Detection, ISO 8802-3

30. Information Processing Systems - Local Area Networks - Part 4: Token-passing Bus Access Method and Physical Layer Specifications, ISO 8802/4.

31. Information Processing Systems - Local Area Networks Part 5: Token Ring Access Method and Physical Layer Specifications, ISO 8802/5.

32. Information Processing Systems - Open Systems Interconnection - Virtual Terminal Services - 
Basic Class, ISO 9040.

33. Information Processing Systems - Open Systems Interconnection - Virtual Terminal Protocol Basic Class, ISO 9041.

34. Information Processing Systems - Open Systems Interconnection. Virtual Terminal Service, Basic Class, ISO 9040, Addendum 1, 1988.

35. Information Processing Systems - Open Systems Interconnection, Virtual Terminal Protocol, Basic Class, ISO 9041, Addendum 1, 1988.

36. Information Processing - Text and Office Systems-Office-Document Architecture (ODA) and Interchange Format - Part 1: Introduction and General Principles, ISO 8613-1.

37. Information Processing - Text and Office Systems-Office-Document Architecture (ODA) and Interchange Format - Part 2: Document Structures ISO 8613-2.

38. Information Processing - Text and Office Systems-Office-Document Architecture (ODA) and Interchange Format - Part 4: Document Profile, ISO 8613-4.

39. Information Processing - Text and Office Systems-Office-Document Architecture (ODA) and Interchange Format - Part 5: Office Document Interchange Format (ODIF), ISO 8613-5.

40. Information Processing - Text and Office Systems-Office-Document Architecture (ODA) and Interchange Format - Part 6: Character Content Architectures, ISO 8613-6.

41. Information Processing - Text and Office Systems-Office-Document Architecture (ODA) and Interchange Format - Part 7: Raster Graphics Content Architectures, ISO 8613-7.

42. Information Processing - Text and Office Systems-Office-Document Architecture (ODA) and Interchange Format - Part 8: Geometric Graphics Content Architectures, ISO 8613-8.

43. Information Processing Systems - Protocol Identification in the Network Layer, DTR 9577.

44. Information Processing Systems - End System to Intermediate System Routing Exchange Protocol for use with ISO 8473 , ISO 9542.

45. Information Processing Systems - Data Communications -Provision of the OSI Connection-mode Network Service, by Packet Mode Terminal Equipment connected to an Integrated Services Digital Network (ISDN), ISO 9574.

46. Information Processing Systems - Transport Service Definition covering Connectionless Mode Transmission, ISO 8072/ADD.

47. Information Processing Systems - Protocol for Providing the Connectionless Mode Transport Service, ISO 8602.

48. Information Processing Systems - Telecommunications and Information Exchange Between Systems -OSI Routing Framework, ISO/TR 9575.

49. Information Processing Systems - Telecommunications and Information Exchange Between Systems -Intermediate systems to Intermediate system Intra-Domain routing exchange protocol for use in conjunction with the protocol for providing the Connectionless mode Network Service ISO 10589.

50. Information Processing Systems - Text Communication - Remote Operations, Part 1: Model, 
Notation and Service Definition, ISO 9072-1

51. Information Processing Systems - Text Communication - Remote Operations, Part 2: Protocol Specification, ISO 9072-2.

52. Information Processing Systems - Text Communication - Reliable Transfer, Part 1: Model and Service Definition, ISO 9066-1.

53. Information Processing Systems - Text Communication - Reliable Transfer, Part 2: Protocol Specification, ISO 9066-2.

54. Information Processing Systems - Open Systems Interconnection - Service Definition of Common Application Service Elements -Commitment, Concurrency and Recovery, ISO 9804.

55. Information Processing Systems - Open Systems Interconnection - Specification of Protocols for Common Application Service Elements - Commitment Concurrency and Recovery, ISO 9805.

56. Information Processing Systems - Open Systems Interconnection - Connectionless ACSE Protocol to Provide the Connectionless - Mode ACSE Service, ISO 10035.

57. Information Processing Systems - Open Systems Interconnection - Connectionless Presentation Protocol to Provide the Connectionless-Mode Presentation Service, ISO 9576.

58. Information Processing Systems - Open Systems Interconnection - Connectionless Session Protocol to Provide the Connectionless-Mode Session Service, ISO 9548.

59. Information Processing Systems - Open Systems Interconnection - Distributed Transaction Processing - Part 1: Model, ISO 10026-1.

60. Information Processing Systems - Open Systems Interconnection - Distributed Transaction Processing -Part 2: Service, ISO 10026-2.

61. Information Processing Systems - Open Systems Interconnection - Distributed Transaction Processing -Part 3: Protocol, ISO 10026-3.

62. Information Processing Systems - Open Systems Interconnection - Remote Database Access Part 1: Generic Model, Service, and Protocol, ISO 9579-1, 1993.

63. Information Processing Systems - Remote Database Access Part 2: SQL Specialization, ISO 9579-2, 1993.

64. Industrial Automation Systems - Manufacturing Message Specification Part 1: Service Definition, ISO 9506-1.

65. Industrial Automation Systems - Manufacturing Message Specification Part 2: Protocol Specification, ISO 9506-2.

66. Information and Documentation - Search and Retrieve Application Service Definition for Open Systems Interconnection, ISO 10162.

67. Information and Documentation - Search and Retrieve Application Protocol Specification for Open Systems Interconnection, ISO 10163.

68. Information Technology - Database Language - SQL - ISO 9075, 1992.

69. Information and Documentation - International Standard Profile - Application Profile for Search 
and Retrieval - Connection-oriented, ISO SC4 WG4 ALD11.

70. Information Technology - Open Systems Interconnection - The Directory: Overview of Concepts, Models, and Service ISO 9594-1.

71. Information Technology - Open Systems Interconnection - The Directory: Models, ISO 9594-2.

72. Information Technology - Open Systems Interconnection - The Directory: Abstract Service Definition ISO 9594-3.

73. Information Technology - Open Systems Interconnection - The Directory: Procedures for Distributed Operation, ISO 9594-4.

74. Information Technology - Open Systems Interconnection - The Directory: Protocol Specifications, ISO 9594-5.

75. Information Technology - Open Systems Interconnection - The Directory: Selected Attribute Types, ISO 9594-6.

76. Information Technology - Open Systems Interconnection - The Directory: Selected Object Classes, ISO 9594-7.

77. Information Technology - Open Systems Interconnection - The Directory: Authentication Framework, ISO 9594-8.

78. Information Technology - Open Systems Interconnection - The Directory: Replication, ISO 95949.

79. Information Technology - Open Systems Interconnection -Management Information Service Definition -Common Management Information Service Definition, ISO 9595.

80. Information Technology - Open Systems Interconnection -Management Information Protocol Specification - Common Management Information Protocol, ISO 9596-1.

81. Information Technology - Open Systems Interconnection - Structure of Management Information Part 2: Definitions of Management Information, ISO 10165-2.

82. Information Technology - Telecommunications and information exchange between systems Elements of Management Information Related to OSI Network Layer Standards, Revised text for ISO DIS 10733, September 26, 1991.

83. Information Technology - Telecommunications and information exchange between systems Intermediate system to Intermediate system Intra-Domain routing information exchange protocol for use in conjnction with the Protocol for providing the Connectionless-mode Network Service (ISO 8473), ISO 10589: 1992(E).

84. Information Technology - Telecommunications and information exchange between systems Elements of Management Information Related to OSI Transport Layer Standards, ISO JTC1/SC6 N6784, November 7, 1991. (DIS Ballot text for ISO DIS 10737-1)

85. Information Technology - Open Systems Interconnection -Structure of Management Information Part 4: Guidelines for the Definition of Managed Objects, ISO 10165-4.

86. Information Technology - Open Systems Interconnection -Systems Management - Part 1: Object Management Function, ISO 10164-1. 
87. Information Technology - Open Systems Interconnection -Systems Management - Part 2: State Management Function, ISO 10164-2.

88. Information Technology - Open Systems Interconnection -Systems Management - Part 3: Attributes for Representing Relationships, ISO 10164-3.

89. Information Technology - Open Systems Interconnection -Systems Management - Part 4: Alarm Reporting Function, ISO 10164-4.

90. Information Technology - Open Systems Interconnection -Systems Management - Part 5: Event Report Management Function, ISO 10164-5.

91. Information Technology - Open Systems Interconnection -Systems Management - Part 6: Log Control Function, ISO 10164-6.

92. Information Technology - Open Systems Interconnection -Systems Management - Part 7: Security Alarm Reporting Function, ISO 10164-7.

93. Information Technology - Open Systems Interconnection -Service definition for the Association Control Service Element - ADDENDUM 1: Peer-entity authentication during association establishment, ISO 8649 DAD 1.

94. Information Technology - Open Systems Interconnection -Protocol specification for the Association Control Service Element - ADDENDUM 1: Peer-entity authentication during association establishment, ISO 8650 DAD 1.

95. Information Processing Systems - Fiber Distributed Data Interface (FDDI) Part 1: Token Ring Physical Layer Protocol, ISO 9314-1.

96. Information Processing Systems - Fiber Distributed Data Interface (FDDI) Part 2: Token Ring Medium Access Control (MAC), ISO 9314-2.

97. Information Processing Systems - Fiber Distributed Data Interface (FDDI) Part 3: Token Ring Physical Medium Dependent (PMD), ISO 9314-3

98. Information Processing Systems - Fiber Distributed Data Interface (FDDI) Part 4: Token Ring Single Mode Fiber Physical Medium Dependent (SMF-PMD), CD 9314-4.

99. Information Processing Systems - Fiber Distributed Data Interface (FDDI) Part 6: Token Ring Station Management (SMT) Medium Dependent (MAC), CD 9314-6.

100. Information Technology - Telecommunications and information exchange between systems End System Routing Information Exchange Protocol for use in conjunction with ISO 8878, ISO 10030.

101. Protocol Combinations to Provide and Support the OSI Network Service - Provision and Support of the Connectionless Mode Network Service, ISO 8880-3.

102. Information Processing Systems - Telecommunications and Information Exchange Between Systems -Protocol for Exchange of Inter-Domain Routing Information among Intermediate Systems to Support Fowarding of ISO 8473 PDUs, CD 10747.

103. Information Processing Systems- Telecommunications and Information Exchange between Systems - MAC Sublayer Interconnection (MAC Bridging), ISO 10038.

104. Information Processing Systems - Open Systems Interconnection - International Standardized 
Profile 12061 - Transaction Processing.

a) ISO ISP 12061-5, ATP 11: Polarized Application Supported Transactions

b) ISO ISP 12061-7, ATP 21: Unchained Provider Supported Transactions

c) ISO ISP 12061-9 ATP 31: Chained Provider Supported Transactions

105. Standard for the Exchange of Product Model Data (STEP), ISO 10303.

106. Procedures for the operation of OSI Registration Authorities, General Procedures, ISO 9834-1, 1992

107. Codes for the Representation of Names of Countries, ISO 3166, 1988

108. Data Interchange - Structure for the Identification of Organizations, ISO 6523, 1984.

109. Information Processing Systems - Computer Graphics -Metafile for the Storage and Transfer of Picture Description Information, ISO 8632:1992.

110. Information Technology - International Standardized Profile 11187-4 - AVT1n, AVT2n - Virtual Terminal Basic Class - Application Profile - Part 4: AVT23 - S mode Paged Application Profile.

111. Information Technology - International Standardized Profile 10611, Parts 1-5 - AMH1n Message Handling Systems - Common Messaging.

112. Information Technology - International Standardized Profile 11188-3 - Common Upper Layer Requirements - Part 3- Minimal OSI Upper Layer Facilities.

113. Information Processing Systems - Open Systems Interconnection Conformance Testing Methodology and Framework, Parts 1-5, ISO IS 9646.

114. General Requirements for the Competence of Calibration and Testing Laboratories, ISO/IEC Guide 25, 1990.

115. General Requirements for the Acceptance of Testing Laboratories, ISO/IEC Guide 38, 1983.

116. Quality Management and Quality Assurance Standards - Guidelines for Selection and Use, ISO/IEC 9000, First Edition March 15, 1987.

The above documents may be obtained from:

ANSI Sales Department
1430 Broadway
New York, NY 10018
(212) $642-4900$

\section{International Telephone and Telegraph Consultative Committee}

1. CCITT Recommendation X.25-1984, Interface Between Data Terminal Equipment (DTE) and Data Circuit-Terminating Equipment (DCE) for Terminals Operating in the Packet Mode on Public Data Networks.

2. CCITT Recommendation X.400, (Red Book, 1984), Message Handling Systems: System Model-Service Elements.

3. CCITT Recommendation X.401, (Red Book, 1984), Message Handling Systems: Basic Service Elements and Optional User Facilities. 
4. CCITT Recommendation X.408, (Red Book, 1984), Message Handling Systems: Encoded Information Type Conversion Rules.

5. CCITT Recommendation X.409, (Red Book, 1984), Message Handling Systems: Presentation Transfer Syntax and Notation.

6. CCITT Recommendation X.410, (Red Book, 1984), Message Handling Systems: Remote Operations and Reliable Transfer Server.

7. CCITT Recommendation X.411, (Red Book, 1984), Message Handling Systems: Message Transfer Layer.

8. CCITT Recommendation X.420, (Red Book, 1984), Message Handling Systems: Interpersonal Messaging User Agent Layer.

9. CCITT Recommendation X.430, (Red Book, 1984), Message Handling Systems: Access Protocol for Teletex Terminals.

10. CCITT Recommendation X.214, (Red Book, 1984), Transport Service Definition for Open Systems Interconnection for CCITT Applications.

11. CCITT Recommendation X.224, (Red Book, 1984), Transport Protocol Specification for Open Systems Interconnection for CCITT Applications.

12. CCITT Recommendation X.215 (Red Book, 1984), Session Service Definition for Open Systems Interconnection for CCITT Applications.

13. CCITT Recommendation X.225 (Red Book, 1984), Session Protocol Specification for Open Systems Interconnection for CCITT Applications.

14. CCITT Recommendation X.400 - Series Implementor's Guide (Version 6, November 1987).

15. CCITT Recommendation X.121 (Red Book, 1985), International Numbering Plan for Public Data Networks.

16. CCITT Recommendation V.35 - Data Transmission at 48 kilobits/second using $60-108 \mathrm{kHz}$ group band circuits.

17. CCITT Recommendation T.410 (Blue Book, 1988) Open Document Architecture (ODA) and Interchange Format - Overview

18. CCITT Recommendation T.411 (Blue Book, 1988) Open Document Architecture (ODA) and Interchange Format - Introduction and General Principles

19. CCITT Recommendation T.412 (Blue Book, 1988) Open Document Architecture (ODA) and Interchange Format - Document Structures

20. CCITT Recommendation T.414 (Blue Book, 1988) Open Document Architecture (ODA) and Interchange Format - Document Profile

21. CCITT Recommendation T.415 (Blue Book, 1988) Open Document Architecture (ODA) and Interchange Format - Document Interchange Format (ODIF)

22. CCITT Recommendation T.416 (Blue Book, 1988) Open Document Architecture (ODA) and Interchange Format - Character Content Architectures 
23. CCITT Recommendation T.417 (Blue Book, 1988) Open Document Architecture (ODA) and Interchange Format - Raster Graphics Content Architectures.

24. CCITT Recommendation T.418 (Blue Book, 1988) Open Document Architecture (ODA) and Interchange Format - Geometric Graphics Content Architectures.

25. CCITT Recommendation Q.921 (I.441) (Blue Book, 1988) ISDN User-Network Interface Data Link Layer Specification.

26. CCITT Recommendation Q.931 (I.451) (Blue Book, 1988) ISDN User-Network Interface Layer 3 Specification for Basic Call Control.

27. CCITT Recommendation X.31 (Blue Book, 1988) Support of Packet Mode Terminal Equipment by an ISDN.

28. CCITT Recommendation X.400 (Blue Book, 1988), Message Handling System and Service Overview. This Recommendation is identical to CCITT Recommendation F.400 (Blue Book, 1988).

29. CCITT Recommendation X.402 (Blue Book, 1988), Message Handling Systems: Overall Architecture.

30. CCITT Recommendation X.403 (Blue Book, 1988), Message Handling Systems: Conformance Testing.

31. CCITT Recommendation X.407 (Blue Book, 1988), Message Handling Systems: Abstract Service Definition Conventions.

32. CCITT Recommendation X.408 (Blue Book, 1988), Message Handling Systems: Encoded Information Type Conversion Rules.

33. CCITT Recommendation X.411 (Blue Book, 1988), Message Handling Systems: Message Transfer System: Abstract Service Definition and Procedures.

34. CCITT Recommendation X.413 (Blue Book, 1988), Message Handling Systems: Message Store: Abstract Service Definition.

35. CCITT Recommendation X.419 (Blue Book, 1988), Message Handling Systems: Protocol Specifications.

36. CCITT Recommendation X.420 (Blue Book, 1988), Message Handling Systems: Interpersonal Messaging System.

37. CCITT Recommendation X.500 (December 1992), Information Technology - Open System Interconnection - The Directory: Concepts, Models and Services (Technically aligned with ISO 9594-1).

38. CCITT Recommendation X.501 (December 1992), Information Technology - Open System Interconnection - The Directory: Models (Technically aligned with ISO 9594-2).

39. CCITT Recommendation X.509 (December 1992), Information Technology - Open System Interconnection - The Directory: Authentication Framework (Technically aligned with ISO 95948).

40. CCITT Recommendation X.511 (December 1992), Information Technology - Open System 
Interconnection - The Directory: Abstract Service Definition (Technically aligned with ISO 95943).

41. CCITT Recommendation X.518 (December 1992), Information Technology - Open System Interconnection - The Directory: Procedures for Distributed Operation (Technically aligned with ISO 9594-4).

42. CCITT Recommendation X.519 (December 1992), Information Technology - Open System Interconnection - The Directory: Protocol Specifications (Technically aligned with ISO 9594-5).

43. CCITT Recommendation X.520 (December 1992), Information Technology - Open System Interconnection - The Directory: Selected Attribute Types (Technically aligned with ISO 9594-6).

44. CCITT Recommendation X.521 (December 1992), Information Technology - Open System Interconnection - The Directory: Selected Object Classes (Technically aligned with ISO 9594-7).

45. CCITT Recommendation X.525 (December 1992), Information Technology - Open System Interconnection - The Directory: Replication (Technically aligned with ISO 9594-9).

46. CCITT Recommendation F.435 - Message Handling Systems: EDI Messaging Service (1991).

47. CCITT Recommendation X.435 - Message Handling Systems: EDI Messaging System (1991).

48. CCITT Draft Recommendation (M.gnm) Generic Network Information Model, November 1991.

49. CCITT Recommendation X.660 (1992), Procedures for the Operation of OSI Registration Authorities, General Procedures.

The above documents may be obtained from: International Telecommunications Union, Place des Nations, CH I2II, Geneve 20 SWITZERLAND.

\section{National Communications System}

Federal Standard FED-STD 1041, Interface Between Data Terminal Equipment (DTE) and Data CircuitTerminating Equipment (DCE) For Operation With Packet-Switched Data Communications Networks, National Communications System.

\section{Standards Council of Canada}

1. CAN-P-1510C, Guidelines for Preparing an Application for Accreditation for Calibration and Testing Laboratories.

2. CAN-P-1511, Guidelines for Preparing a Quality Manual for Testing Organizations.

3. CAN-P-1515, Conditions for the Accreditation of Calibration and Testing Laboratories.

4. CAN-P-4C, General Requirements for the Accreditation of Calibration and Testing Laboratories.

5. PALCAN, Information Technology and Telecommunications (IT\&T) Laboratory Accreditation Program, Version 1.0.

6. PALCAN, Information Technology and Telecommunications (IT\&T) Laboratory Accreditation Program - Checklist for the assessor, SCC 1003-15, Version 1.0.

The above documents may be obtained from: the Standards Council of Canada, 1200 - 45 O'Connor Street, Ottawa, Ontario, K1P 6N7, Canada. 


\section{National Institute of Standards and Technology}

1. NIST Special Publication 500-206, Stable Implementation Agreements for Open Systems Interconnection Protocols, Version 6, December 1992. This document can be purchased from National Technical Information Service (NTIS), U.S. Department of Commerce, 5285 Port Royal Road, Springfield, VA 22161. For telephone orders call: (703) 487-4650. For information on how to access this document on-line, contact Brenda Gray at (301) 975-3664.

2. FIPS Pub 107, Local Area Networks: Baseband Carrier Sense Multiple Access with Collision Detection Access Method and Physical Layer Specifications and Link Layer Protocol, NTIS, U.S. Department of Commerce, 5285 Port Royal Road, Springfield, VA 22I6I.

3. FIPS Pub 100-1, Interface Between Data Terminal Equipment (DTE) and Data Circuit-Terminating Equipment (DCE) For Operation With Packet-Switched Data Networks, (PDSN), or between two DTEs, by Dedicated Circuit. NTIS, U.S. Department of Commerce, 5285 Port Royal Road, Springfield, VA 2216I.

4. FIPS Pub 179, Government Network Management Profile (GNMP). See [NIST 7] for ordering information.

5. Military Supplement to ISO Transport Protocol, National Institute of Standards and Technology, National Computer Systems Laboratory, ICST/SNA-85-17, 1985.

6. Implementation Guide for ISO Transport Protocol, National Institute of Standards and Technology, National Computer Systems Laboratory, ICST/SNA-85-18, 1985.

7. NIST Special Publication 500-192 Government Open Systems Interconnection User's Guide, Version 2. This document can be purchased from the National Technical Information Service (NTIS), U. S. Department of Commerce, 5285 Port Royal Road, Springfield, VA 22161. For telephone orders call (703) 487-4650.

8. GOSIP Conformance and Interoperation Testing and Registration, March 1991, Version 1.0, NISTIR 4594. The document can be obtained from NIST, Systems \& Network Architecture Division, Automated Methods Group, Bldg 225, Room B217, Gaithersburg, MD 20899.

9. NIST Special Publication 500-182, Guidelines for the Evaluation of Message Handling Systems Implementations. See [NIST 7] for NTIS ordering information.

10. NIST Special Publication 500-196, Guidelines for the Evaluation of File Transfer, Access and Management Implementations. See [NIST 7] for NTIS ordering information.

11. FIPS Pub 128, Computer Graphics Metafile (CGM).

12. FIPS Pub 152, Information Processing - Text and office systems - Standard Generalized Markup Language (SGML) - Aligned with ISO 8879.

13. Working Implementation Agreements for Open Systems Interconnection Protocols, March 1992.

14. "Specifications for a Secure Hash Standard", proposed Federal Information Processing Standard, January 22, 1992 (available from NIST, call (301-975-2816)

15. NIST Special Publication 500-205, Guidelines for the Evaluation of Virtual Terminal Implementation. See [NIST 7] for NTIS ordering information.

16. NVLAP Handbook, Operational Requirements of the Laboratory Accreditation Program of Computer Applications Testing for GOSIP Conformance Testing, September 1991. NVLAP, 
NIST, Gaithersburg, MD 20899 USA. New version will supersede this handbook.

17. The Government Open Systems Interconnection Profile, FIPS PUB 146, U.S. Department of Commerce, National Institute of Standards and Technology, Gaithersburg MD, August 1988.

18. The Government Open Systems Interconnection Profile, FIPS PUB 146-1, U.S. Department of Commerce, National Institute of Standards and Technology, Gaithersburg MD, April 1991.

19. Favreau, J.P.; Mills, K.L.; Nightingale, J.S., The U.S. GOSIP Testing Program, Proc. of the 6th International Conference on the Application of Standards for Open Systems, October 1990, Washington D.C., U.S.A.

20. Nightingale S.; Mills K.L.; Favreau, J.P., The U.S. GOSIP Testing Program, Progress and Outlook, Proceedings of the Worldwide Recognition of OSI Test Results Workshop, Gaithersburg, U.S.A., May 1991.

21. Nightingale, J.S., GOSIP Conformance and Interoperation Testing and Registration, NISTIR 4594, U.S. Department of Commerce, National Institute of Standards and Technology, Gaithersburg MD, March 1991.

22. Nightingale, J.S., GOSIP Means of Testing Validation, 1993. GOSIP Testing Program, NIST, Building 225 Room B217, Gaithersburg, MD 20899 USA.

23. Nightingale, J.S., GOSIP Product Registration Criteria, March 1993. GOSIP Testing Program, NIST, Building 225 Room B217, Gaithersburg, MD 20899 USA.

24. Favreau, J.P., The Five Q's (Cues) of the U.S. GOSIP Testing Program, Participant's Proceedings of the GOSIP Procurement Symposium. December 7, 1992. NIST, Building 225 Room B217, Gaithersburg, MD 20899 USA.

25. NIST Special Publication 500-217, Industry/Government Open Systems Specification - Version 1. This document can be purchased from National Technical Information Service (NTIS), U.S. Department of Commerce, 5285 Port Royal Road, Springfield, VA 22161, order number PB91. 171967. For telephone orders call: (703) 487-4650. This document may also be purchased from the IEEE Computer Society, Order Department, phone: 1-800-272-6657.

\section{National Voluntary Laboratory Accreditation Program}

1. NVLAP Handbook, Operational Requirements of the Laboratory Accreditation Program of Computer Applications Testing for GOSIP Conformance Testing, September 1991. NVLAP, NIST, Gaithersburg, MD 20899 USA. New version will supersede this handbook.

2. NIST Handbook 150, National Voluntary Laboratory Accreditation Program, Procedures and General Requirements, March 1994.

The above documents may be obtained from: the National Voluntary Laboratory Accreditation Program, National Institute of Standards and Technology, Giathersburg, MD 20899.

\section{Joint Interoperability Test Center}

1. Means of Testing validation, Part 1-3, Defense Information Systems Agency, Joint Interoperability and Engineering Organization, Joint Interoperability Test Center, Fort Huachuca, Arizona. April 1993.

\section{Miscellaneous}


1. Manufacturing Automation Protocol

2. Technical and Office Protocols, Specification Version 3.0

For copies of the two documents listed above, contact: Corporation for Open Systems, 8260 Willow Oaks Corporate Drive, Suite 700, Fairfax, VA 22031.

3. RFC 1331, Point-to-Point Protocol for the transmission of multi-protocol datagrams over Pointto-Point links, D. Perkins, July 1990.

4. RFC 1237 Guidelines for OSI NSAP allocation in the Internet, R. Colella, E.P. Gardner, R.W. Callon, July 1991.

5. RFC 1172, The Point-to-Point Protocol (PPP) Initial Configuration Options, D. Perkins, R. Hobby

6. RFC 1377, The PPP OSI Network Layer Control Protocol (OSINLCP), November 1992.

Copies of the four documents listed above can be obtained from the Internet Network Information Center (NIC).

7. X-Window System, X Version 11 R4 (X11-R4).

8. Z39.50 Implementors Group Profile, ANSI Z39.50-MA-026.

9. Network Management Forum: Forum 006, "Forum Library-Volume 4: OMNIPoint 1 Definitions," Issue 1.0, August 1992. 
